
\title{
25 Research Suare \\ Fosl1 Targets AMPK to Inhibit Autophagy-Mediated Anti-Apoptotic and Anti-Inflammatory Effects in Spinal Cord Injury
}

\section{Lin Zhong}

The First Affiliated Hospital of Anhui Medical University

\section{Sheng Fang}

First Affiliated Hospital of Anhui Medical University

\section{An-Quan Wang}

The First Affiliated Hospital of Anhui Medical University

\section{Tao Wang}

Changzheng Hospital: Shanghai Changzheng Hospital

\section{Wei Huang}

The First Affiliated Hospital of USTC: Anhui Provincial Hospital

\section{Zheng-Jun Pan}

the third affiliated hospital of anhui medical university

\section{Hui Zhang}

the first affiliated hospital of anhui medical university

\section{Zongsheng Yin ( $\sim$ yinzongshengahmu@126.com )}

The first affiliated hospital of anhui medical university

\section{Research}

Keywords: Fos like antigen 1 (Fosl1), AMP-activated protein kinase (AMPK), autophagy, inflammation, apoptosis, spinal cord injury (SCl)

Posted Date: November 18th, 2020

DOI: https://doi.org/10.21203/rs.3.rs-105918/v1

License: (c) (i) This work is licensed under a Creative Commons Attribution 4.0 International License. Read Full License 


\section{Abstract}

Background: The objective of this study was to delineate the role of Fosl 1 in regulating inflammation and apoptosis following spinal cord injury.

Methods: GSE45006 datasets from Gene Expression Omnibus (GEO) were explored to analyze Fosl1 gene expression. Next, we established an animal model to assess Fosl1 and AMPK by western blotting, realtime PCR, and immunohistochemical staining and used immunofluorescence staining to check Fosl 1 expression in neurons. Fosl1 silencing was used to assess the effect on AMPK, cell viability, autophagy, inflammation and apoptosis. Subsequently, an AMPK activator and inhibitor were added to PC-12 cells with $\mathrm{H}_{2} \mathrm{O}_{2}$-induced injury subjected to si-Fosl 1 treatment to examine the change in the above indexes and to determine whether the benefits from Fosl1 silencing occurred via AMPK. Moreover, we employed chloroquine (CQ) and rapamycin (RAP) to activate and inhibit autophagy, respectively, and revealed the effects of the upregulation and downregulation of autophagy following AMPK interference. Finally, an animal model was used to identify the effect of si-Fosl1 in vivo.

Results: Based on the analysis of the GSE45006 datasets, Fosl1 was found to be highly expressed and was also found to be significantly enhanced in our animal model. Fosl1 knockdown upregulated AMPK at the protein and mRNA levels, promoted autophagic proteins (LC3 II/I, Beclin1) and inhibited inflammatory factors (IL-1 $\beta$, IL-6, TNF- $\alpha$ ) and apoptosis markers (caspase3, Bax). However, Fosl1 decreased the negatively related autophagic protein p62, the anti-inflammatory factor IL-10 and the anti-apoptotic marker Bcl-2. By utilizing compound C (com, an AMPK inhibitor), we learned that AMPK inhibition exhibited unfavorable effects on autophagy but promoted inflammation and apoptosis following Fosl1 silencing. AMPK activation showed contrasting effects. Moreover, we used CQ (an autophagic inhibitor), which indicated that CQ reversed the benefits of AMPK activation on inflammation and apoptosis. The autophagic activator RAP attenuated the negative effects after com treatment. In vivo, si-Fosl1 increased BBB scores at $7 \mathrm{~d}$ and $14 \mathrm{~d}$ and motor neurons, meanwhile, it decreased the number of apoptotic cells, and inflammatory cytokine expression at $14 \mathrm{~d}$ postoperation.

Conclusion: Fosl 1 can suppress AMPK to promote inflammation and apoptosis through autophagy in $\mathrm{SCl}$.

\section{Background}

Spinal cord injury (SCl) is a disastrous nerve trauma in the central nervous system (CNS) currently without sufficient treatment options, which affects several millions people worldwide and leads to catastrophic complications [1, 2], such as neurological deficits [3], paraplegia [4], osteoporosis [5], and others. These complications place huge psychological and physical burdens on patients and threaten their health and life quality [6]. Despite strenuous efforts that have been spent on a treatment strategy for $\mathrm{SCI}[7,8]$ and that considerable progress has been exhibited in trials, satisfactory clinical benefits still remain unavailable [9]. In order to explore more effective therapies, an understanding of the mechanism 
and pathology involved in $\mathrm{SCl}$ is critical [10]. Due to abundant harmful factors at the injury site following $\mathrm{SCl}$ and the inability of neuron axon regeneration, $\mathrm{SCl}$ recovery is almost impossible. Since effective treatments against $\mathrm{SCl}$ are not possible, a way to decrease the degree of $\mathrm{SCl}$ has become a research focus. The pathological process of $\mathrm{SCl}$ involves primary injury and secondary injury, and evidence has indicated that the secondary injury is the essential molecular event [11]. Because the primary injury is unpredictable and irreversible, therapeutic interventions for the secondary injury to reduce damage, inhibit apoptosis, and alleviate the inflammatory response during the acute period of $\mathrm{SCl}$ represents a novel research strategy for SCI [12-14].

Fos like antigen 1 (Fosl1) is a transcription factor of the fos gene family that has been researched extensively in many diseases $[15,16]$. It can combine with proteins of the Jun family to mediate tumor occurrence, progression, invasion, metastasis, and other processes [17-20]. In addition, it is particularly related to cell differentiation, proliferation and apoptosis [21] and plays an important role in cholangitis [22], arthritis [23], and diseases of damage, such as neuron injury [24-27]. One study has indicated that Fosl1 deficiency protects mouse embryo fibroblasts from apoptosis induced by oxidative stress [28]. Nevertheless, Fosl1 overexpression enhances the apoptosis of C6 glioma cells [29]. The role of Fosl1 has been investigated in a model of lung injury, where it regulates LPS-induced inflammatory factors such as IL-10 and IL-1 $\beta[26,30,31]$. Fosl 1 is obviously enhanced following injury and increases cell inflammation and apoptosis. However, whether it also has a vital role in the secondary injury of $\mathrm{SCl}$ and what that may be are still unclear.

Autophagy is a unique lysosomal-mediated catabolic process in eukaryotic organisms that phagocytizes and degrades abnormal proteins or dysfunctional organelles for maintaining homeostasis in response to different stresses, including nutrient deprivation, injury, and hypoxia [32-34]. It can be activated by internal or external stimuli and reduce the harm of detrimental factors or some metabolic wastes, which become isolated within a bilayer membrane of the autophagosome, accompanied by LC3 II formation from LC3 I [35]. Subsequently, these autophagosomes combine with lysosomes to form autolysosomes, which degrade these substances to recycle them. A number of studies have reported that autophagy is upregulated in many diseases, such as cancers, brain injury, and kidney injury [32]. Multiple studies have shown that autophagy has an important protective role in the secondary injury and recovery of SCl [3638]. Autophagy, which usually maintains a low activity, can be induced following $\mathrm{SCl}$ and exert antiapoptotic and anti-inflammatory effects $[9,39,40]$, but this autophagy-induced effect in SCl is very limited. Therefore, how to enhance autophagy to reduce apoptosis and inflammation is a research focus, but the underlying molecular mediated-mechanism has still not been elucidated clearly. Thus, we assume that the upregulation of Fosl1 promotes inflammation and apoptosis to aggravate the secondary injury via constraining autophagic activity in SCl. AMP-activated protein kinase (AMPK) is a classic signaling pathway that participates in mediating autophagy and is expressed at a basal level in the body [41]. It can be activated in SCl and plays an important protective role in SCl improvement [37, 42]. Hence, we further hypothesized that Fosl 1 amplifies inflammation and apoptosis by inhibiting autophagy via the AMPK signaling pathway following spinal cord injury. 


\section{Methods}

\subsection{Bioinformatic analysis}

To investigate the hypothesis that Fosl1 was upregulated in $\mathrm{SCl}$, we explored the Gene Expression Omnibus database (GSE 45006) and used the GPL1355 platforms to download the information of the sham group and the $\mathrm{SCl} 1 \mathrm{~d}, 3 \mathrm{~d}, 7 \mathrm{~d}$, and $14 \mathrm{~d}$ groups. After analysis with R language, we filtered out the 150 top differential genes in each time point and generated heatmaps. Subsequently, we analyzed the Fosl 1 data to define our time point for the SCl animal model.

\subsection{Animal model, groups and treatments}

Eighteen specific pathogen-free adult Sprague-Dawley (SD) rats weighting 220-250 g were provided by the Anhui Experimental Animal Center of China. Subsequently, the animals were bred in an airconditioned room with a $12 \mathrm{~h}$ day and night cycle under a stable temperature $\left(24 \pm 2^{\circ} \mathrm{C}\right)$ and humidity $(60 \pm 5 \%)$.

In part 1 , the rats were randomly divided into three groups: normal group $(n=6)$, sham group $(n=6)$, and $\mathrm{SCl}$ group $(\mathrm{n}=6)$. The $\mathrm{SCl}$ model was established based on a previously published report using Allen's method [43]. Briefly, following anesthetization, a T10 laminectomy was executed from T8 to T12. Next, rats from the $\mathrm{SCl}$ group were subjected to a force $(10 \mathrm{~g} \times 5 \mathrm{~cm})$ to produce a T10 contusive SCl. The animals in the sham group received a laminectomy only, and those in the normal group received no treatment. The bladder was emptied twice per day until its function was restored.

In part 5, 20 rats were divided into four groups: sham + saline group $(n=5), S C l+$ saline group $(n=5), S C l$ + si-NC group $(n=5)$, and $\mathrm{SCl}+$ si-Fosl1 group. The animals were intrathecally injected with sterile saline, si-NC, or si-Fos 1 immediately and were intramuscularly injected with penicillin for the first 3 consecutive days after the operation. The BBB scale was applied to evaluate the hindlimb movement at the designed time point, and the rats were sacrificed to remove the target tissues after 14 days.

\subsection{Cell culture and treatments}

PC-12 is a classical neuronal cell line that was purchased from the Chinese Academy of Sciences. To further identify the mechanism involved in $\mathrm{SCl}$, we utilized this cell line treated with $\mathrm{H}_{2} \mathrm{O}_{2}(100 \mu \mathrm{mol}$ for $24 \mathrm{~h}$ ) to mimic neuron injury. PC-12 cells were cultured in high-glucose DMEM with a GlutaMAX ${ }^{\mathrm{TM}}$ supplement (10564011, Gibco) and 10\% fetal bovine serum (10100147C, Gibco). Our in vitro study involved three parts (part 2, part 3, and part 4). In part 2, we used si-RNA to verify whether there were benefits following Fosl1 knockdown. In this part, the cells were divided into four groups: $P B S, \mathrm{H}_{2} \mathrm{O}_{2}, \mathrm{H}_{2} \mathrm{O}_{2}$ + si-NC, and $\mathrm{H}_{2} \mathrm{O}_{2}+$ si-RNA. To check whether the benefits of Fosl1 knockdown occurred through the AMPK signaling pathway, we designed another set of groups in part 3: $\mathrm{H}_{2} \mathrm{O}_{2}+$ si-RNA, $\mathrm{H}_{2} \mathrm{O}_{2}+$ si-RNA + metformin (met, an activator, $20 \mathrm{mmol}$ for $24 \mathrm{~h}$ ) [44], and H2O2 + si-RNA + compound C (com, an inhibitor, $2 \mu \mathrm{mol}$ for $24 \mathrm{~h}$ ) [33]. In part 4, rapamycin (RAP, an activator of autophagy, $5 \mu \mathrm{mol}$ for $24 \mathrm{~h}$ ) and chloroquine (CQ, an inhibitor of autophagy, $20 \mu \mathrm{mol}$ for $24 \mathrm{~h}$ ) [45] were used to assess whether the effect 
of the Fosl1 knockdown was generated ultimately via autophagy. The cells were grouped as follows: $\mathrm{H}_{2} \mathrm{O}_{2}+$ si-RNA + met VS. $\mathrm{H}_{2} \mathrm{O}_{2}+$ si-RNA + met + CQ, $\mathrm{H}_{2} \mathrm{O}_{2}+$ si-RNA + com VS. and $\mathrm{H}_{2} \mathrm{O}_{2}+$ si-RNA + com + RAP.

\subsection{Hindlimb locomotor assessment}

The Basso, Beattie, and Bresnahan (BBB) scale [46] was applied to evaluate hindlimb locomotion. After surgery, 0 score represented complete hindlimb disability, which indicated that the $\mathrm{SCl}$ model was successful. The test was performed by three researchers who were blinded to the specifics of the three groups. Finally, the scores were averaged.

\subsection{Nissl staining and TUNEL staining}

The rats were sacrificed for removing the spinal cord. The horizontal tissue sections were harvested.

To count the motor neurons, Nissl staining (Beyotime, Shanghai, China) was used to evaluate the Nissl bodies. After staining with crystal violet for $10 \mathrm{~min}$, the tissue sections were observed to count the Nissl bodies in 5 randomly chosen fields with a microscope at a magnification of 200x (Olympus, Japan).

The TUNEL Apoptosis Assay Kit (Beyotime, Shanghai, China) was used to assess cell apoptosis in the specimens according to the manufacturer's protocol. After staining, the samples were observed with an Olympus immunofluorescence microscope to calculate the number of TUNEL + cells.

\subsection{Immunofluorescence (IF) staining}

To identify that transcription factor Fosl1 was expressed in neurons, immunofluorescence double-label staining was employed to check whether Fosl1 (1:100, sc-28310, Santa Cruz Biotechnology) and $\beta$-III tubulin (neuronal marker protein) $(1: 100,5568 T$, Cell Signaling Technology) co-localized in neuronal cells.

To assess the inflammatory level of the spinal cord after si-Fosl1 injection, immunofluorescence staining was applied to examine the inflammatory marker protein. The sections were incubated with primary antibody in a refrigerator for $24 \mathrm{~h}$ and then with secondary antibody and DAPI (Beyotime, Shanghai, China) at room temperature. Next, the sections were assessed as described above.

\subsection{Immunohistochemistry (IHC) staining}

To detect histological changes in Fosl1 (1:100, sc-28310, Santa Cruz Biotechnology) and AMPK (1:100, sc74461, Santa Cruz Biotechnology), the slides were stained by IHC. After treatment with hydrogen peroxide, QuickBlock ${ }^{\mathrm{TM}}$ Blocking Buffer (Beyotime, Shanghai, China) was used to block non-specific proteins. The primary antibodies were applied to cover the tissue at $4{ }^{\circ} \mathrm{C}$ overnight, and then the tissues were incubated with secondary antibody (ZSGB-BIO, Beijing, China). Following staining with DAB (ZSGBBIO, Beijing, China) and hematoxylin (Beyotime, Shanghai, China), the slides were visualized using an Olympus microscope.

\subsection{Western blotting analysis (WB)}


The spinal cord was extracted and dissolved in RIPA lysis buffer (Beyotime, Shanghai, China) to which protease and phosphatase inhibitors were added. The mixture was incubated on ice for $30 \mathrm{~min}$ and centrifuged at $12000 \mathrm{rpm}$ for $10 \mathrm{~min}$, after which the supernatant was collected. Total protein was measured using bicinchoninic acid assays in accordance with the manufacturer's instructions and the concentration was uniform. Subsequently, $5 \times$ SDS loading buffer was added to the supernatant for denaturing the sample. The samples were separated by SDS-PAGE using the same volume per sample. Next, the proteins were transferred to a PVDF membrane (Millipore, MA, USA). After blocking with $5 \%$ skimmed milk, the membrane was incubated overnight at $4{ }^{\circ} \mathrm{C}$ with the following primary antibodies: Fosl1 (1:500, sc-28310, Santa Cruz Biotechnology), AMPK (1:800, sc74461, Santa Cruz Biotechnology), pAMPK (1:800, 50081S, Cell Signaling Technology), LC3 A/B (1:800, 4108S, Cell Signaling Technology), p62 (1:800, 23214S, Cell Signaling Technology), Beclin1 (1:800, 3495T, Cell Signaling Technology), cleaved caspase3 (1:500, 9664S, Cell Signaling Technology), Bax (1:800, sc-7480, Santa Cruz Biotechnology), Bcl-2 (1:800, sc-7382, Santa Cruz Biotechnology), $\beta$-actin (1:2000, AB2837817, Affinity Biosciences), and GAPDH (1:2000, 5174T, Cell Signaling Technology). The next day, the membrane was washed with TBST three times, was subsequently immersed in the corresponding secondary antibody (1:20000, AB2839429/AB2839430, Affinity Biosciences), and then incubated with ECL (Thermo Scientific, USA). The bands were checked using an imaging system (Bio-Rad, USA), and the IOD value was measured by Image-Pro Plus software.

\subsection{Quantitative real-time PCR (qRT-PCR)}

Total RNA was extracted from the spinal cord or cells using TRIzol reagent (Invitrogen, USA). The concentration of mRNA was examined using a NanoDrop One device (Thermo, MA, USA) and diluted to $0.5 \mu \mathrm{g} / \mu \mathrm{l}$. The purity was assessed using $260 / 280 \mathrm{~nm}$. Following RNase-free DNase digestion, a reverse transcription kit was used for cDNA synthesis (TaKaRa, Tokyo, Japan). The primers were synthesized by Sangon Biotech Co., Ltd. according to the Pubmed Gene Bank (Table 1).

Table 1

Primers sequence used for qRT-PCR.

\begin{tabular}{|lll|}
\hline Gene & Sequence $\left(\mathbf{5}^{\prime} \mathbf{-} \mathbf{3}^{\prime}\right)$ & Size $(\mathbf{b p})$ \\
\hline Fosl1 & Forward: CGACCAGGAGTCATACGAGC & 140 \\
\hline & Reverse: CGGTTTCTGCACTTAGCAGC & \\
\hline AMPK & Forward: GCAGTTGCCTACCACCTCAT & 88 \\
\hline & Reverse: AAGAATCGGGTGGGCTTGTT & \\
\hline GAPDH & Forward: AGTGCCAGCCTCGTCTCATA & 133 \\
\hline & Reverse: ATGAAGGGGTCGTTGATGGC \\
\hline
\end{tabular}

A $20 \mu$ reaction system was used as follows: $1 \mu \mathrm{l} \mathrm{cDNA,} 1 \mu$ f forward primer, $1 \mu$ reverse primer, $10 \mu \mathrm{l} 2 \times$ mix and $7 \mu \mathrm{l}$ RNase-free water. The PCR protocol included $95^{\circ} \mathrm{C}$ for $2 \mathrm{~min}$, followed by 40 cycles of $95^{\circ} \mathrm{C}$ 
for $10 \mathrm{~s}$ and $60^{\circ} \mathrm{C}$ for $30 \mathrm{~s}$. GAPDH was used as the standard reference. All experiments were performed in triplicate, and the experimental data were analyzed by the 2- $\triangle \triangle \mathrm{CT}$ method.

\subsection{Enzyme-Linked Immunosorbent Assay (ELISA)}

After the cell samples were homogenized with 0.1 M PBS on ice, the mixture was centrifuged at $5000 \mathrm{~g}$ for $10 \mathrm{~min}$. The supernatant was removed to another tube for a subsequent assessment. Enzyme-Linked Immunosorbent Assay Kits (IL-1 $\beta$, IL-6, IL-10, and TNF-a) were provided by Abcam (Cambridge, UK). The optical density values at $450 \mathrm{~nm}$ were recorded. All procedures were performed in accordance with the manufacturer's protocol.

\subsection{Small interfering RNA (si-RNA) transfection}

To knockdown Fosl 1 expression, PC-12 cells were cultured in a 6 -well plate at $1 \times 10^{5}$ cells/well in highglucose DMEM without FBS and transiently transfected with si-Fosl1 with a working concentration of 50 nM using a riboFECTTM CP Transfection Kit (RiboBio, Guangzhou, China) according to the manufacturer's instructions. The sequence used was 5'-CATCGAAAGAGTAGCAGCA-3'. Immunofluorescence microscopy (Olympus, Japan) and qRT-PCR were employed to monitor the efficiency $48 \mathrm{~h}$ after transfection.

\subsection{Cell Counting Kit-8 (CCK-8)}

The Enhanced Cell Counting Kit-8 (Beyotime, Shanghai, China) was used to monitor the cell viability. After the cells $\left(100 \mu \mathrm{l}\right.$ of a $5 \times 10^{4}$ cells/ml suspension) were seeded in 96 -well plates for $24 \mathrm{~h}$, reagents or PBS were added to the plates for the cells' treatments. Subsequently, $10 \mu$ CCK-8 solution was added to the 96-well plates. After incubation, the 96 -well plate was examined at $450 \mathrm{~nm}$ in an instrument to detect the enzyme label at each time point.

\subsection{Flow cytometry (FCM)}

Flow cytometry was used to assess the cell apoptosis rate after the different treatments. After digestion with $0.25 \%$ trypsin without EDTA, the cells were harvested in an EP tube and washed three times with precooled PBS. The precipitates were resuspended into a single cell suspension in $100 \mu l \times$ buffer, after which $5 \mu$ l annexin V-FITC and $5 \mu$ PI staining solution (Vazyme Biotech, Nanjing, China) were added. Subsequently, another $400 \mu \mathrm{l} 1 \times$ buffer was added to these mixtures, after which the cell apoptosis rate was examined using a flow cytometer.

\subsection{CO-immunoprecipitation (COIP)}

To further investigate the relationship between Fosl1 and AMPK, we employed COIP to examine whether there was a direct interaction between them. Cells were collected and treated by IP-grade Cell Lysis Buffer (Beyotime, Shanghai, China) for 10 min on ice. This mixture was added into a $1.5 \mathrm{ml}$ EP tube for centrifugation (12000 rpm, $4^{\circ} \mathrm{C}, 10 \mathrm{~min}$ ). Subsequently, $10 \mu \mathrm{l}$ of the mixture was retained as the input. 
The remainder was transferred to another EP tube to which a pre-treating solution including $1 \mu \mathrm{gg}$ IgG and 20 ul Protein A/G PLUS-Agarose (Santa Cruz, CA, USA) was added for removal of non-specific proteins. After centrifugation, the supernatant was transferred to three other EP tubes, to which was added IgG, an anti-Fosl 1 antibody, or an anti-AMPK antibody, respectively, in addition to 20 ul Protein A/G PLUSAgarose, followed by incubation at $4{ }^{\circ} \mathrm{C}$ overnight. Following centrifugation, the pellets were rinsed three times with lysis buffer and boiled for $10 \mathrm{~min}$. The samples were detected using western blotting.

\subsection{Statistical analysis}

All results were presented as the mean \pm standard error of the mean $(M \pm S E M)$. The difference between two groups was analyzed using an unpaired 2-tailed t-test. One-way analysis of variance (ANOVA) with the least significant difference (LSD) and Student-Newman-Keuls (S-NK) tests were used to make comparisons among multiple groups with SPSS 21.0 (SPSS, Inc., Chicago, IL, USA). The graphs were generated with GraphPad Prism 6.02 (GraphPad Software, Inc., California, USA). A p $<0.05$ was considered to be statistically significant.

\section{Results}

3.1 Fosl 1 was significantly upregulated at $1 \mathrm{~d}$ and $3 \mathrm{~d}$ postoperation, as assessed by a bioinformatic analysis of the Gene Expression Omnibus database.

Based on extraction and analysis of data from the GSE 45006 datasets, which included $20 \mathrm{SCl}$ rats and 4 sham rats, we collected the information of the sham group and the SCl groups (at $1 d, 3 d, 7 d$, and $14 d$ postoperation) (Fig. 1A) and used the R language tool to analyze these data. One hundred fifty differentially expressed genes were found, which are shown in the four heatmaps (Fig. 1B). Fosl1 was highly expressed at $1 \mathrm{~d}$ and $3 \mathrm{~d}$ postoperation compared to the sham group, but there was no significance between the groups at $7 \mathrm{~d}$ and $14 \mathrm{~d}$ postoperation (Fig. 1C).

3.2 Fosl 1 and AMPK were obviously upregulated in the spinal cord at $1 \mathrm{~d}$ postoperation, and Fosl 1 was also expressed significantly in neurons.

According to the bioinformatic analysis results, $1 \mathrm{~d}$ was chosen as the time point for sacrificing animals and harvesting the target tissues. After investigating Fosl1 protein expression in the three groups, we found that Fosl 1 were upregulated significantly in the injured spinal cord vs. the other groups, and the comparison between the normal group and the sham group was not significantly different (Fig. 2B). To examine the expression more specifically, qRT-PCR was used to examine the Fos 11 mRNA level. Similar to what was seen with the western blotting, it was observed that Fosl1 mRNA in the SCl group was expressed highly vs. the other groups (Fig. 2C). There was also no significant difference between the normal group and the sham group at the mRNA level (Fig. 2C). To assess the histological change, we used immunohistochemical staining, which showed that the average optical density of Fosl 1 was significantly greater compared to the other groups (Fig. 3A). 
To further examine Fosl1 expression in neurons, we employed immunofluorescence double-label staining to detect Fosl1 and the neuronal biomarker protein $\beta$-III tubulin, which demonstrated that Fos 11 was highly expressed in $\beta$-III tubulin-positive cells (Fig. 2F).

AMPK was examined using western blotting, qRT-PCR and immunohistochemical staining as described above. We determined that AMPK was increased obviously at the mRNA and protein levels (Fig. 2D, E), and there was a significant difference in the histological behavior (Fig. 3B).

\subsection{Fosl1 and AMPK were enhanced significantly in PC-12 cells with $\mathrm{H}_{2} \mathrm{O}_{2}$-induced injury.}

To further examine Fosl1 and AMPK expression, the classical $\mathrm{H}_{2} \mathrm{O}_{2}$-induced PC-12 injury model was employed to mimic neuronal cell injury. We found that the Fosl1 expressions at mRNA and protein level in the $\mathrm{H}_{2} \mathrm{O}_{2}$ group was increased apparently compared to the PBS group (Fig. 4B, C). Its expression in the PBS group was negligible. The same methods were used to investigate AMPK expression at the protein and mRNA levels. We discovered a similar result compared to Fosl1, with a significant difference between both groups (Fig. 4D, E).

\subsection{Knockdown of Fosl1 enhanced AMPK expression and autophagic activity in $\mathrm{PC}-12$ cells with $\mathrm{H}_{2} \mathrm{O}_{2}$-induced injury.}

To further detect Fosl1 function, si-RNA technology was used to knockdown Fosl1 expression. We found that Fosl1 was downregulated in the si-Fosl1 group compared to the $\mathrm{H}_{2} \mathrm{O}_{2}$ group and the $\mathrm{H}_{2} \mathrm{O}_{2}+$ si-NC group (Fig. 4B, C), but the AMPK and p-AMPK expression levels were enhanced vs. both groups (Fig. 4D, E). COIP was employed to identify whether there is a connection between Fosl 1 and AMPK. The result indicated Fosl 1 directly bound to AMPK in injured PC-12 cells (Fig. 5C). Autophagy is a normal response to external or internal stimuli. To assess autophagic activity, marker proteins such as LC3 II/I, Beclin1, and p62 were tested. We found that the positively related proteins LC3 II/I and Beclin1 were highly upregulated in the si-Fosl 1 group, and the negatively related protein p 62 was decreased vs. the $\mathrm{H}_{2} \mathrm{O}_{2}$ group and the $\mathrm{H}_{2} \mathrm{O}_{2}+$ si-NC group (Fig. 5A).

3.5 Knockdown of Fosl1 improved the survival rate and decreased inflammation and apoptosis in PC-12 cells with $\mathrm{H}_{2} \mathrm{O}_{2}$-induced injury.

To check the effect of Fosl1 knockdown on injured PC-12 cells, CCK-8 and FCM was applied to assess the cell viability and the apoptotic rate. We learned that the cell viability in the si-Fos 1 group was higher than in the other two groups (Fig. 4A), but the apoptotic rate was lower (Fig. 5B). In addition, inflammation was assessed using ELISA technology. The pro-inflammatory proteins IL-1 $\beta$, IL-6, and TNF-a were attenuated significantly vs. the $\mathrm{H}_{2} \mathrm{O}_{2}$ group and the $\mathrm{H}_{2} \mathrm{O}_{2}+$ si-NC group, but the protective protein IL-10 was increased (Fig. 5D). To investigate apoptosis, we performed western blotting to assess the Bcl-2, Bax, and caspase3 
proteins. The results showed that the apoptotic-inducing proteins Bax and caspase 3 were decreased in the si-Fosl1 group and the apoptotic-inhibiting protein Bcl-2 was enhanced (Fig. 5A).

3.6 Upregulation of AMPK could increase cell viability and autophagic activity, and alleviate inflammation and apoptosis in injured PC-12 cells with Fosl1 knockdown. Inactivated AMPK could neutralize the beneficial effects of si-Fosl1 in injured PC-12 cells.

To investigate the detailed role of AMPK in injured PC-12 cells after si-Fosl 1 treatment, an AMPK activator (met) was used to enhance the AMPK activity (Fig. 6B). We found that LC3 II/I, and Beclin1 were increased and that p62 was downregulated (Fig. 7A), which indicated that the autophagic level was enhanced significantly in the met group following AMPK activation. The proteins positively related to inflammation and apoptosis, such as IL-1 $\beta$, IL-6, TNF-a, Bax, and caspase3, were decreased (Fig. 6C, 7A). Nevertheless, the anti-inflammatory cytokine IL-10 and the anti-apoptotic protein Bcl-2 were increased (Fig. 6C, 7A). The cell viability of met group was better than control group (Fig. 6A), but the apoptotic rate was lower (Fig. 7B).

The same method was applied to inhibit AMPK activity using com. By inactivating AMPK, we observed that the cell viability and autophagic activity were obviously downregulated (Fig. 6A, 7B). However, the levels of the inflammatory and apoptotic levels were enhanced (Fig. 6C, 7A).

3.7 Autophagic activation could attenuate inflammation and apoptosis in injured PC-12 cells with Fosl1 knockdown after AMPK inhibition, but autophagic suppression exhibited the opposite effects.

To identify whether AMPK-mediated inflammation and apoptosis in injured PC-12 were regulated via autophagy, we divided the injured PC-12 cells with si-Fosl1 treatment into two groups. One group had cells treated with AMPK activator (met) and autophagic inhibitor (CQ), and the other group was treated with AMPK inhibitor (com) and autophagic activator (RAP). We found that CQ alleviated the AMPK activator benefits on inflammation and apoptosis (Fig. 8B, C). Meanwhile, CQ decreased the cell viability and raised apoptotic cells rate (Fig. 8A, D).

The autophagic activator prevented inflammation and apoptosis after com treatment (Fig. 9B, C). moreover, RAP increased the cell viability and reduced apoptotic cells rate (Fig. 9A, D). These outcomes further illustrated that AMPK-mediated inflammation and apoptosis occurred via autophagy.

\section{8 si-Fosl1 improved neurological function in rats with SCl.}

To verify the si-Fosl 1 benefits on rats with spinal cord injury, we designed four groups (sham + saline, $\mathrm{SCl}$ + saline, $\mathrm{SCl}+\mathrm{si}-\mathrm{NC}$, and $\mathrm{SCl}+\mathrm{si}-\mathrm{Fos} / 1$ ). The BBB scale was employed to evaluate the hindlimb function at 30 min pre-operation and at $0 \mathrm{~d}, 1 \mathrm{~d}, 3 \mathrm{~d}, 7 \mathrm{~d}$, and $14 \mathrm{~d}$ postoperation. The preoperative BBB score of all animals and at each time point in the sham group was 21 , but the score in the other groups was 0 at $0 \mathrm{~d}$, which showed that the rat SCI model was successful. Additionally, there was no significant difference when comparing the $\mathrm{SCl}+$ si-Fosl 1 group with the $\mathrm{SCl}+$ saline group and the $\mathrm{SCl}+\mathrm{si}-\mathrm{NC}$ group at 1 and 3 
d (Fig. 10A). The results were significantly different, which indicated that si-Fosl1 could evidently improve the hindlimb locomotion at 7 and $14 \mathrm{~d}$ postoperation (Fig. 10A). A comparison between the $\mathrm{SCl}+$ saline group and the $\mathrm{SCl}+$ si-NC group showed that there was no significant difference at each time point (Fig. 10A). This result further demonstrated that Fosl1 knockdown could promote the recovery of neurological function and improve the hindlimb locomotion.

\section{9 si-Fosl1 decreased apoptosis and inflammation but increased the survival rate of motor neurons.}

To further check the difference at the cellular level, TUNEL and Nissl staining were used to examine the apoptotic cells and the surviving motor neurons, respectively. By analyzing the TUNEL + cells, we found that there were significant differences between the $\mathrm{SCl}+$ si-Fosl1 group and the other two groups (Fig. 10B, E). To further assess the neurological function of the hindlimb at histologic level, we used Nissl staining at $14 \mathrm{~d}$ following $\mathrm{SCl}$. The Nissl bodies were markedly increased in the $\mathrm{SCl}+\mathrm{si}$-Fosl 1 group (Fig. 10C, F).

The inflammatory change in the four groups was assessed using immunofluorescence staining at $14 \mathrm{~d}$ postoperation. By measuring the reactive astrocytes marker GFAP expression which reflects inflammatory activity, we found that the $\mathrm{SCl}+\mathrm{si}$-Fosl1 group fared lower than the $\mathrm{SCl}+$ saline group and the $\mathrm{SCl}+\mathrm{si}-\mathrm{NC}$ group (Fig. 10D, G). Moreover, the comparison between the other two groups showed that there was no significant difference (Fig. 10D, G). Subsequently, the inflammatory marker proteins, IL-1 $\beta$, IL-6, IL-10, TNF-a were checked. The results indicated the inflammation level was lower in the $\mathrm{SCl}+\mathrm{si}$-Fosl1 group than the other two groups (Fig. 11A-H).

\section{Discussion}

Spinal cord injury remains an unsolved neurologic disease with no effective clinical treatment to date, although considerable progress has been made in the past few decades [47]. The main challenge in the field of $\mathrm{SCl}$ is the massive loss of neurons during secondary injury and the extremely limited neuronal regeneration $[48,49]$. Innovations in stem cell transplantation once presented an effective strategy to provide extrinsic nerve cells to build new networks and rescue neurological function, but satisfactory outcomes have yet to be been achieved [50]. The SCl secondary injury plays a more important role in the neuronal loss, which is often accompanied by inflammation, apoptosis, autophagy, and necrosis [51, 52]. However, the precise mechanism is still unclear.

Although Fosl 1 is well-known as an essential transcription factor that constitutes the AP-1 compound with c-fos and c-jun in the development of many diseases of injury [24, 53-55], its role in the occurrence and progression of SCl has not yet been elucidated. By exploring the GSE45006 and analyzing the datasets, we learned that Fosl 1 expression was significantly upregulated following SCl at $1 \mathrm{~d}$ and $3 \mathrm{~d}$ postoperation. In our study, we verified that Fosl1 was highly expressed at the mRNA and protein levels at $1 \mathrm{~d}$ postoperation. The outcome was consistent with previous studies from the GEO database. Due to 
neuronal injury playing an essential role in $\mathrm{SCl}$, we used double-label immunofluorescence staining to detect Fos 1 in neurons and discovered that Fosl 1 expression was significantly enhanced in neurons. In addition, we used $100 \mathrm{nmol} / \mathrm{L} \mathrm{H}_{2} \mathrm{O}_{2}$ to induce injury in PC-12 cells for $24 \mathrm{~h}$ to mimic neuronal injury in vitro. Fosl 1 was also observed to be highly expressed at the mRNA and protein levels. These results indicated that the upregulation of Fosl 1 in neurons may play an essential role during secondary injury in vivo and vitro.

Small interfering RNA is a double-stranded RNA with a specific length and sequence that completely complements a specific mRNA and results in degradation of the target mRNA and blocking of the translation process $[56,57]$. We used si-RNA technology to precisely assess the Fosl1 role in SCl. After Fosl1 knockdown in the injured neurons, the cells' viability was increased significantly, but the cells' apoptotic rate was decreased. Additionally, we found that the pro-inflammatory cytokines IL-1 $\beta$, IL- 6 and TNF-a were downregulated and that the anti-inflammatory cytokine IL-10 was upregulated following Fosl1 knockdown. These outcomes indicated that Fosl1 silencing is advantageous for inhibiting neuronal injury. In addition, the proteins positively related to autophagy and apoptosis, such as Beclin1, LC3 II/I and $\mathrm{Bcl}-2$, were enhanced, but negatively related proteins, such as p62, Bax, and caspase3, were reduced. Moreover, the signaling pathway proteins AMPK and p-AMPK were increased. These results clearly clarified that si-Fosl 1 can activate AMPK and autophagy and can alleviate neuronal inflammation and apoptosis.

The AMPK signaling pathway is a classical pathway that is involved in modulating autophagy via threonine (Thr-172) phosphorylation [58]. A previous study has shown that AMPK was increased and improved function in a rat model with $\mathrm{SCl}$ [59]. This result was shown at the protein and mRNA levels in our study. To determine the detailed role of AMPK in injured neurons, we used its activator (metformin, met) and inhibitor (compound C, com) to treat the injured cells after si-Fosl1 treatment [60-62]. After met utilization for $24 \mathrm{~h}$, we found that the apoptotic rate was lower and that the cell viability was also improved. The inflammatory cytokines and apoptotic proteins were diminished significantly, although IL10 and $\mathrm{Bcl}-2$ were upregulated. These interesting phenomena were completely opposite after com treatment, and more importantly, the autophagy activity was decreased. These results suggested that siFosl1 exerts anti-inflammatory and anti-apoptotic effects via the AMPK signaling pathway.

Autophagy is regarded as an innate immune defense mechanism against a more serious external or internal injury [63], which performs an anti-inflammatory and anti-apoptotic role in various diseases [6466]. A previous study showed that inhibition of autophagy can increase neuronal apoptosis and synapsis stabilization in mice [67]. To identity whether these benefits of AMPK upregulation on cell injury following si-Fosl1 treatment worked through autophagy, we used CQ [68] to inhibit the autophagic activity after AMPK activator treatment in injured PC-12 cells transfected with si-Fosl1. We demonstrate that inflammatory and apoptotic proteins were highly expressed after $C Q$ treatment and that the cells' viability and apoptotic rate were also worsened. These results exhibited that the si-Fosl1 benefits through the AMPK signaling pathway can be neutralized by autophagic depression. Moreover, autophagic activator (RAP) [69] was employed to activate autophagy following AMPK inhibition. It was of great interest to 
observe that RAP could reverse the inflammation and apoptosis after AMPK inactivity in injured cells with si-Fosl 1 treatment and benefit the cells' viability and apoptotic rate. Combining these results, we were able to definitely conclude that Fosl 1 suppressed AMPK activity to activate inflammation and apoptosis through autophagy in SCl. Our findings are the first to demonstrate the novel axis of Fosl1/AMPK/autophagy playing a vital role in $\mathrm{SCl}$.

Next, we utilized an animal model to verify that si-Fosl 1 also exerts beneficial effects in vivo. The BBB scores showed that si-Fosl 1 improved the hindlimb locomotor function at $7 \mathrm{~d}$ and $14 \mathrm{~d}$ postoperation, but there was no significance at $1 \mathrm{~d}$ and $3 \mathrm{~d}$ postoperation. The apoptotic cells change was evaluated using TUNEL staining at $14 \mathrm{~d}$, which certified that TUNEL + cells was obviously lower than that in the control groups. In addition, Nissl staining was used to count the motor neurons and we found that the number of Nissl bodies was significantly better in si-Fosl 1 group. Moreover, immunofluorescence staining was used to examine inflammatory marker cytokines (IL-1 $1 \beta, \mathrm{IL}-6, \mathrm{IL}-10, \mathrm{TNF}-\mathrm{a}$ ), and inflammatory cytokines expression in the both control groups were worse than that in the si-Fosl 1 group, however, the antiinflammatory cytokine IL-10 was higher. Meanwhile, the reactive astrocytes marker (GFAP) was lowly expressed in the si-Fos 11 group. These results revealed that si-Fosl 1 exerted anti-inflammatory and antiapoptotic effects on $\mathrm{SCl}$ in vitro.

\section{Conclusion}

In conclusion, our study is the first to show that Fosl1 plays a vital role in aggravating $\mathrm{SCl}$ secondary injury and that Fosl 1 knockdown can promote autophagy-mediated anti-apoptotic and anti-inflammatory effects to improve neurological function via the AMPK signaling pathway.

\section{Abbreviations}

Fosl1: Fos like antigen 1 AMPK:AMP-activated protein kinase; GEO:Gene Expression Omnibus; CQ:chloroquine; RAP:rapamycin; IL-1 $\beta$ :interleukin-1 $\beta$; TNF-a:tumor necrosis factor- $\alpha$; SCl:spinal cord injury; CNS:central nervous system; BBB:Basso, Beattie, and Bresnahan scale; TUNEL:TdT-mediated dUTP NickEnd Labeling; IF:Immunofluorescence staining; qRT-PCR:Quantitative real-time PCR; ELISA:Enzyme-Linked Immunosorbent Assay; si-RNA:Small interfering RNA; CCK-8:Cell Counting Kit-8; FCM:Flow cytometry; COIP:CO-immunoprecipitation; NC:Non-specific control; GFAP:Glial fibrillary acidic protein.

\section{Declarations}

\section{Acknowledgments}

We would like to thank the Key Lab of Gene Resource Utilization for Genetic Diseases of Ministry of Education and the Provincial Laboratory of Microbiology and Parasitology Anhui.

\section{Author Contributions}


ZSY, LZ and HZ designed the experiment; LZ, SF, AQW completed the experiments; LZ, TW, WH analyzed the data; LZ and ZSY writed and edited the manuscript. All authors drafted and reviewed the manuscript and approved the final version to be published.

\section{Funding}

This work was supported by the National Natural Science Foundation of China (No. 81672161 and No. 81871785) and the Provincial Natural Science Foundation of Anhui (No. 2008085MH247).

\section{Availability of data and materials}

The datasets used and/or analyzed during the current study are available from the corresponding author on reasonable request.

\section{Ethics approval and consent to participate}

All experimental procedures were performed in accordance with the National Institutes of Health $(\mathrm{NIH})$ Guide for the Care and Use of Laboratory Animals. Animal experiments were approved by the Ethics Committee of Anhui Medical University of China.

\section{Consent for publication}

Not applicable.

\section{Competing interests}

The authors declare that they have no competing interests.

\section{Author details}

1 Department of Orthopedics, the First Affiliated Hospital of Anhui Medical University, NO. 218 Jixi Road, Hefei, 230022, China

2 Department of Orthopedics, the Third Affiliated Hospital of Anhui Medical University, NO. 390 Huaihe Road, Hefei, 230061, China

3 Spine Tumor Center, Department of Orthopedic Oncology, Changzheng Hospital, Second Military Medical University, NO. 415 Fengyang Road, Shanghai, 200003 China

4 Department of Orthopaedics, The First Affiliated Hospital of USTC, Division of Life Sciences and Medicine, University of Science and Technology of China, NO. 17 Lujiang Road, Hefei, 230001, China

\section{References}


1. Silva NA, Sousa N, Reis RL, Salgado AJ. From basics to clinical: a comprehensive review on spinal cord injury. Prog Neurobiol. 2014;114:25-57.

2. Singh A, Tetreault L, Kalsi-Ryan S, Nouri A, Fehlings MG. Global prevalence and incidence of traumatic spinal cord injury. Clin Epidemiol. 2014;6:309-31.

3. Bravo-Esteban E, Taylor J, Aleixandre M, et al. Longitudinal estimation of intramuscular Tibialis Anterior coherence during subacute spinal cord injury: relationship with neurophysiological, functional and clinical outcome measures. J Neuroeng Rehabil. 2017;14(1):58.

4. Zhang Q, Huang C, Meng B, Tang T, Shi Q, Yang H. Acute effect of Ghrelin on ischemia/reperfusion injury in the rat spinal cord. Int J Mol Sci. 2012;13(8):9864-76.

5. Haider IT, Lobos SM, Simonian N, Schnitzer TJ, Edwards WB. Bone fragility after spinal cord injury: reductions in stiffness and bone mineral at the distal femur and proximal tibia as a function of time. Osteoporos Int. 2018;29(12):2703-15.

6. McDonald JW, Sadowsky C. Spinal-cord injury. Lancet. 2002;359(9304):417-25.

7. Chen J, Joon Lee H, Jakovcevski I, et al. The extracellular matrix glycoprotein tenascin-C is beneficial for spinal cord regeneration. Mol Ther. 2010;18(10):1769-77.

8. Nakagawa H, Ninomiya T, Yamashita T, Takada M. Treatment With the Neutralizing Antibody Against Repulsive Guidance Molecule-a Promotes Recovery From Impaired Manual Dexterity in a Primate Model of Spinal Cord Injury. Cereb Cortex. 2019;29(2):561-72.

9. Saraswat Ohri S, Bankston AN, Mullins SA, et al. Blocking Autophagy in Oligodendrocytes Limits Functional Recovery after Spinal Cord Injury. J Neurosci. 2018;38(26):5900-12.

10. Zhou K, Sansur CA, Xu H, Jia X. The Temporal Pattern, Flux, and Function of Autophagy in Spinal Cord Injury. Int J Mol Sci. 2017. 18(2).

11. Penas C, Guzmán MS, Verdú E, Forés J, Navarro X, Casas C. Spinal cord injury induces endoplasmic reticulum stress with different cell-type dependent response. J Neurochem. 2007;102(4):1242-55.

12. Chen XB, Wang ZL, Yang QY, et al. Diosgenin Glucoside Protects against Spinal Cord Injury by Regulating Autophagy and Alleviating Apoptosis. Int J Mol Sci. 2018. 19(8).

13. Bareyre FM. Neuronal repair and replacement in spinal cord injury. J Neurol Sci. 2008. 265(1-2): 6372.

14. Coutts M, Keirstead HS. Stem cells for the treatment of spinal cord injury. Exp Neurol. 2008;209(2):368-77.

15. Bacigalupo I, Palladino C, Leone P, et al. Inhibition of MMP-9 expression by ritonavir or saquinavir is associated with inactivation of the AKT/Fra-1 pathway in cervical intraepithelial neoplasia cells. Oncol Lett. 2017;13(5):2903-8.

16. Hu X, Zhang Z, Liang Z, et al. Downregulation of feline sarcoma-related protein inhibits cell migration, invasion and epithelial-mesenchymal transition via the ERK/AP-1 pathway in bladder urothelial cell carcinoma. Oncol Lett. 2017;13(2):686-94. 
17. Talotta F, Casalino L, Verde P. The nuclear oncoprotein Fra-1: a transcription factor knocking on therapeutic applications' door. Oncogene. 2020;39(23):4491-506.

18. Duong HQ, Nemazanyy I, Rambow F, et al. The Endosomal Protein CEMIP Links WNT Signaling to MEK1-ERK1/2 Activation in Selumetinib-Resistant Intestinal Organoids. Cancer Res. 2018;78(16):4533-48.

19. Leconet W, Chentouf M, du Manoir S, et al. Therapeutic Activity of Anti-AXL Antibody against TripleNegative Breast Cancer Patient-Derived Xenografts and Metastasis. Clin Cancer Res. 2017;23(11):2806-16.

20. Cho H, Matsumoto S, Fujita Y, et al. Trametinib plus 4-Methylumbelliferone Exhibits Antitumor Effects by ERK Blockade and CD44 Downregulation and Affects PD-1 and PD-L1 in Malignant Pleural Mesothelioma. J Thorac Oncol. 2017;12(3):477-90.

21. Trop-Steinberg S, Azar Y. AP-1 Expression and its Clinical Relevance in Immune Disorders and Cancer. Am J Med Sci. 2017;353(5):474-83.

22. Kireva T, Erhardt A, Tiegs G, et al. Transcription factor Fra-1 induces cholangitis and liver fibrosis. Hepatology. 2011;53(4):1259-69.

23. Hannemann N, Cao S, Eriksson D, et al. Transcription factor Fra-1 targets arginase-1 to enhance macrophage-mediated inflammation in arthritis. J Clin Invest. 2019;129(7):2669-84.

24. Nishina T, Komazawa-Sakon S, Yanaka S, et al. Interleukin-11 links oxidative stress and compensatory proliferation. Sci Signal. 2012;5(207):ra5.

25. Lu Z, Miao Z, Zhu J, Zhu G. ETS-domain containing protein (Elk1) suppression protects cortical neurons against oxygen-glucose deprivation injury. Exp Cell Res. 2018;371(1):42-9.

26. Rajasekaran S, Tamatam CR, Potteti HR, et al. Visualization of Fra-1/AP-1 activation during LPSinduced inflammatory lung injury using fluorescence optical imaging. Am J Physiol Lung Cell Mol Physiol. 2015;309(4):L414-24.

27. Mutoh J, Ohsawa M, Hisa H. Effect of naloxone on ischemic acute kidney injury in the mouse. Neuropharmacology. 2013;71:10-8.

28. Vaz M, Machireddy N, Irving A, et al. Oxidant-induced cell death and Nrf2-dependent antioxidative response are controlled by Fra-1/AP-1. Mol Cell Biol. 2012;32(9):1694-709.

29. Shirsat NV, Shaikh SA. Overexpression of the immediate early gene fra- 1 inhibits proliferation, induces apoptosis, and reduces tumourigenicity of c6 glioma cells. Exp Cell Res. 2003;291(1):91100.

30. Vaz M, Reddy NM, Rajasekaran S, Reddy SP. Genetic disruption of Fra-1 decreases susceptibility to endotoxin-induced acute lung injury and mortality in mice. Am J Respir Cell Mol Biol. 2012;46(1):5562.

31. Mishra RK, Potteti HR, Tamatam CR, Elangovan I, Reddy SP. C-Jun Is Required for Nuclear Factor-KBDependent, LPS-Stimulated Fos-Related Antigen-1 Transcription in Alveolar Macrophages. Am J Respir Cell Mol Biol. 2016;55(5):667-74. 
32. Kroemer G, Mariño G, Levine B. Autophagy and the integrated stress response. Mol Cell. 2010;40(2):280-93.

33. Zhao H, Chen S, Gao K, et al. Resveratrol protects against spinal cord injury by activating autophagy and inhibiting apoptosis mediated by the SIRT1/AMPK signaling pathway. Neuroscience. 2017;348:241-51.

34. Egan D, Kim J, Shaw RJ, Guan KL. The autophagy initiating kinase ULK1 is regulated via opposing phosphorylation by AMPK and mTOR. Autophagy. 2011;7(6):643-4.

35. Eskelinen EL. Maturation of autophagic vacuoles in Mammalian cells. Autophagy. 2005;1(1):1-10.

36. Kanno H, Ozawa H, Sekiguchi A, Itoi E. Spinal cord injury induces upregulation of Beclin 1 and promotes autophagic cell death. Neurobiol Dis. 2009;33(2):143-8.

37. Zhou K, Zheng Z, Li Y, et al. TFE3, a potential therapeutic target for Spinal Cord Injury via augmenting autophagy flux and alleviating ER stress. Theranostics. 2020;10(20):9280-302.

38. Vahsen BF, Ribas VT, Sundermeyer J, et al. Inhibition of the autophagic protein ULK1 attenuates axonal degeneration in vitro and in vivo, enhances translation, and modulates splicing. Cell Death Differ. 2020.

39. Wang ZY, Lin JH, Muharram A, Liu WG. Beclin-1-mediated autophagy protects spinal cord neurons against mechanical injury-induced apoptosis. Apoptosis. 2014;19(6):933-45.

40. Netea-Maier RT, Plantinga TS, van de Veerdonk FL, Smit JW, Netea MG. Modulation of inflammation by autophagy: Consequences for human disease. Autophagy. 2016;12(2):245-60.

41. Zhang Q, Yang YJ, Wang $\mathrm{H}$, et al. Autophagy activation: a novel mechanism of atorvastatin to protect mesenchymal stem cells from hypoxia and serum deprivation via AMP-activated protein kinase/mammalian target of rapamycin pathway. Stem Cells Dev. 2012;21(8):1321-32.

42. Ohtake $Y$, Sami A, Jiang $X$, et al. Promoting Axon Regeneration in Adult CNS by Targeting Liver Kinase B1. Mol Ther. 2019;27(1):102-17.

43. Zhong L, Zhang H, Ding ZF, et al. Erythropoietin-Induced Autophagy Protects Against Spinal Cord Injury and Improves Neurological Function via the Extracellular-Regulated Protein Kinase Signaling Pathway. Mol Neurobiol. 2020.

44. Inyang KE, Szabo-Pardi T, Wentworth E, et al. The antidiabetic drug metformin prevents and reverses neuropathic pain and spinal cord microglial activation in male but not female mice. Pharmacol Res. 2019;139:1-16.

45. Zhu HL, Xu XF, Shi XT, et al. Activation of autophagy inhibits cadmium-triggered apoptosis in human placental trophoblasts and mouse placenta. Environ Pollut. 2019;254(Pt A):112991.

46. Basso DM, Beattie MS, Bresnahan JC. A sensitive and reliable locomotor rating scale for open field testing in rats. J Neurotrauma. 1995;12(1):1-21.

47. Yang L, Conley BM, Cerqueira SR, et al. Effective Modulation of CNS Inhibitory Microenvironment using Bioinspired Hybrid-Nanoscaffold-Based Therapeutic Interventions. Adv Mater. 2020: e2002578. 
48. Lou J, Lenke LG, Ludwig FJ, O'Brien MF. Apoptosis as a mechanism of neuronal cell death following acute experimental spinal cord injury. Spinal Cord. 1998;36(10):683-90.

49. Fakhoury M. Spinal cord injury: overview of experimental approaches used to restore locomotor activity. Rev Neurosci. 2015;26(4):397-405.

50. Yuan T, Liu Q, Kang J, Gao H, Gui S. High-Dose Neural Stem/Progenitor Cell Transplantation Increases Engraftment and Neuronal Distribution and Promotes Functional Recovery in Rats after Acutely Severe Spinal Cord Injury. Stem Cells Int. 2019. 2019: 9807978.

51. Wang J, Rong Y, Ji C, et al. MicroRNA-421-3p-abundant small extracellular vesicles derived from M2 bone marrow-derived macrophages attenuate apoptosis and promote motor function recovery via inhibition of mTOR in spinal cord injury. J Nanobiotechnology. 2020;18(1):72.

52. Jiang D, Gong F, Ge X, et al. Neuron-derived exosomes-transmitted miR-124-3p protect traumatically injured spinal cord by suppressing the activation of neurotoxic microglia and astrocytes. J Nanobiotechnology. 2020;18(1):105.

53. Huang C, Dai D, Xie H, et al. Lgr4 Governs a Pro-Inflammatory Program in Macrophages to Antagonize Post-Infarction Cardiac Repair. Circ Res. 2020.

54. Pozas E, Aguado F, Ferrer I. Fra-1 immunoreactivity in the rat brain during normal postnatal development and after injury in adulthood. Neurosci Res. 1999;33(2):137-45.

55. Xu X, Jiang R, Gong P, et al. Up-regulation of FOS-like antigen 1 contributes to neuronal apoptosis in the cortex of rat following traumatic brain injury. Metab Brain Dis. 2018;33(1):115-25.

56. Irie A, Sato K, Hara RI, Wada T, Shibasaki F. An artificial cationic oligosaccharide combined with phosphorothioate linkages strongly improves siRNA stability. Sci Rep. 2020;10(1):14845.

57. Ly S, Echeverria D, Sousa J, Khvorova A. Single-Stranded Phosphorothioated Regions Enhance Cellular Uptake of Cholesterol-Conjugated siRNA but Not Silencing Efficacy. Mol Ther Nucleic Acids. 2020;21:991-1005.

58. Bruiners N, Dutta N, Guerrini V, et al. The anti-tubercular activity of simvastatin is mediated by cholesterol-driven autophagy via the AMPK-mTORC1-TFEB axis. J Lipid Res. 2020.

59. Wang P, Xie ZD, Xie CN, et al. AMP-activated protein kinase-dependent induction of autophagy by erythropoietin protects against spinal cord injury in rats. CNS Neurosci Ther. 2018;24(12):1185-95.

60. Wang C, Yao Z, Zhang Y, et al. Metformin Mitigates Cartilage Degradation by Activating AMPK/SIRT1-Mediated Autophagy in a Mouse Osteoarthritis Model. Front Pharmacol. 2020;11:1114.

61. Kim YM, Kim MY, Kim HJ, et al. Compound C independent of AMPK inhibits ICAM-1 and VCAM-1 expression in inflammatory stimulants-activated endothelial cells in vitro and in vivo. Atherosclerosis. 2011;219(1):57-64.

62. Zhang D, Xuan J, Zheng BB, et al. Metformin Improves Functional Recovery After Spinal Cord Injury via Autophagy Flux Stimulation. Mol Neurobiol. 2017;54(5):3327-41.

63. Wang Z, Zhou L, Zheng X, et al. Autophagy protects against PI3K/Akt/mTOR-mediated apoptosis of spinal cord neurons after mechanical injury. Neurosci Lett. 2017;656:158-64. 
64. Kan LL, Liu D, Chan BC, et al. The flavonoids of Sophora flavescens exerts anti-inflammatory activity via promoting autophagy of Bacillus Calmette-Guérin-stimulated macrophages. J Leukoc Biol. 2020.

65. Kim JH, Sim HA, Jung DY, et al. Poria cocus Wolf Extract Ameliorates Hepatic Steatosis through Regulation of Lipid Metabolism, Inhibition of ER Stress, and Activation of Autophagy via AMPK Activation. Int J Mol Sci. 2019. 20(19).

66. Das LM, Binko AM, Traylor ZP, Peng H, Lu KQ. Vitamin D improves sunburns by increasing autophagy in M2 macrophages. Autophagy. 2019;15(5):813-26.

67. Catalani E, Bongiorni S, Taddei AR, et al. Defects of full-length dystrophin trigger retinal neuron damage and synapse alterations by disrupting functional autophagy. Cell Mol Life Sci. 2020.

68. Huang W, Cheng C, Shan WS, et al. Knockdown of SGK1 alleviates the IL-1 $\beta$-induced chondrocyte anabolic and catabolic imbalance by activating Fox01-mediated autophagy in human chondrocytes. FEBS J. 2020;287(1):94-107.

69. Harada M, Hanada S, Toivola DM, Ghori N, Omary MB. Autophagy activation by rapamycin eliminates mouse Mallory-Denk bodies and blocks their proteasome inhibitor-mediated formation. Hepatology. 2008;47(6):2026-35.

\section{Figures}

A Time points of

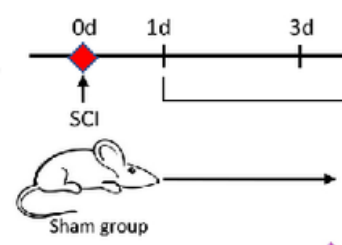

B

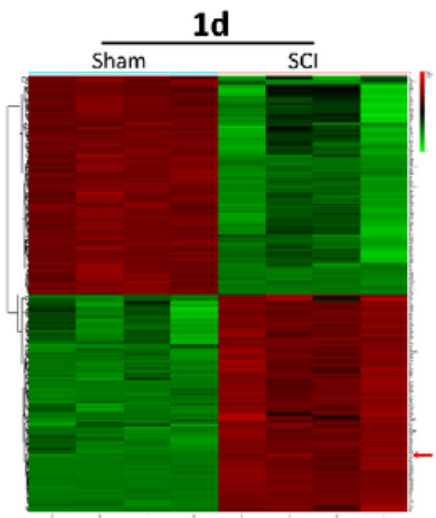

d
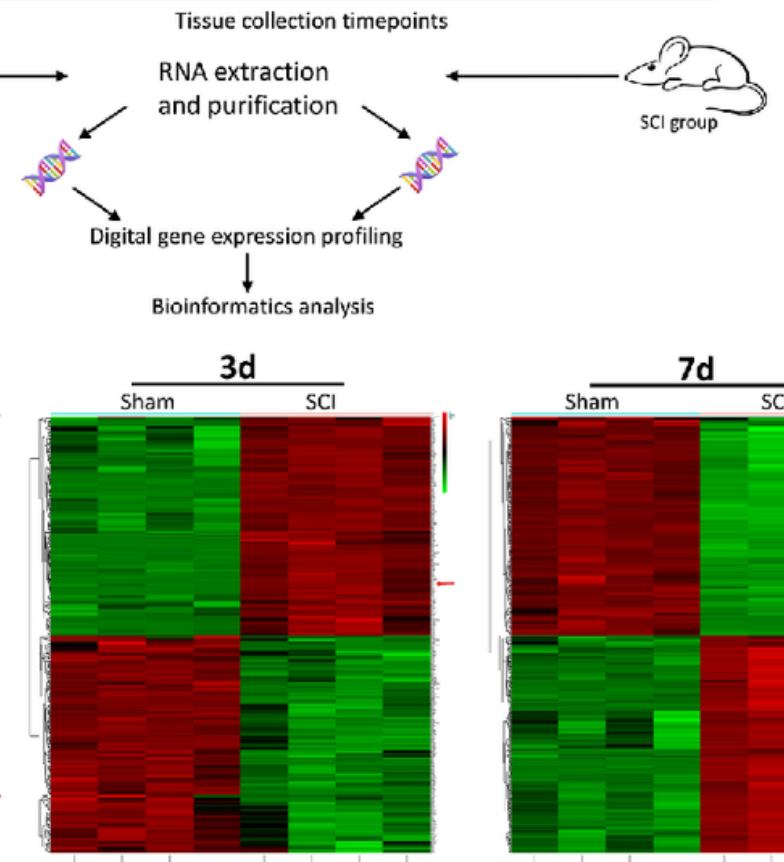

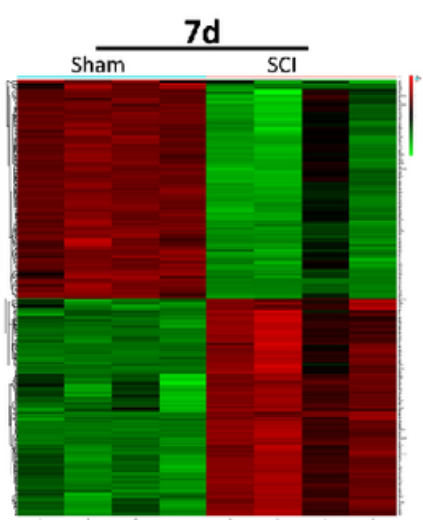

c
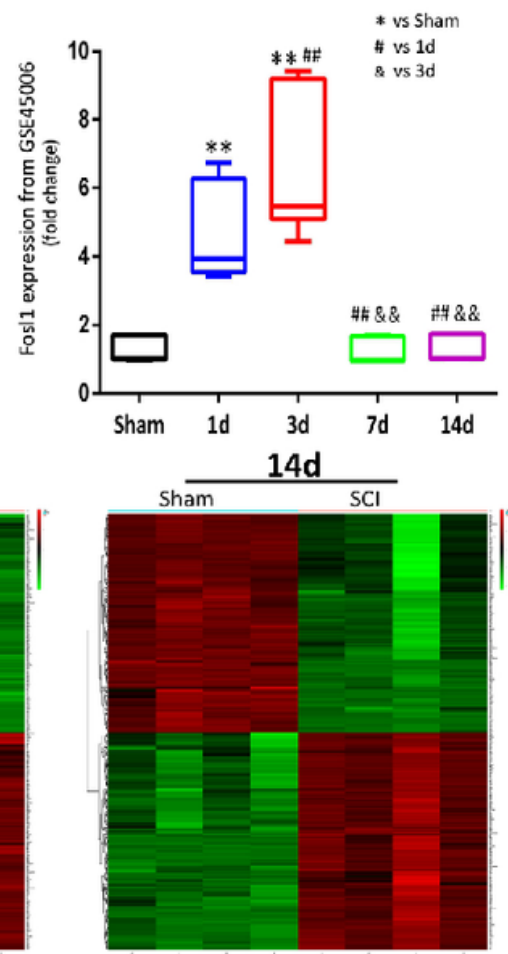

\section{Figure 1}


The mRNA of Fosl 1 was highly expressed in rats following $\mathrm{SCl}$ at $1 \mathrm{~d}$ and $3 \mathrm{~d}$ postoperation. A: Schematic of the experimental strategy used to screen the potential differential genes in the GEO database. B: The heatmaps generated by $\mathrm{R}$ language using the mRNA expression data of the $\mathrm{SCl}$ group and the sham group in GEO at $1 \mathrm{~d}, 3 \mathrm{~d}, 7 \mathrm{~d}$, and $14 \mathrm{~d}$ postoperation. C: The Fos 1 mRNA expression (normalized to that of the sham group) in the spinal cord of rats suffering from SCl at $1 \mathrm{~d}, 3 \mathrm{~d}, 7 \mathrm{~d}$, and 14 d postoperation $(n=4)$. All data are presented as the $M \pm S E M$. ${ }^{*} p<0.05$ vs. sham group, ${ }^{* \star} p<0.01$ vs. sham group, $\# p<0.05$ vs. $S C l$ group at $1 \mathrm{~d}$ postoperation, \#\#p<0.01 vs. $S C l$ group at $1 \mathrm{~d}$ postoperation, \&p<0.05 vs. $\mathrm{SCl}$ group at $3 \mathrm{~d}$ postoperation, $\& \& \mathrm{p}<0.01 \mathrm{vs}$. $\mathrm{SCl}$ group at $3 \mathrm{~d}$ postoperation by ANOVA.

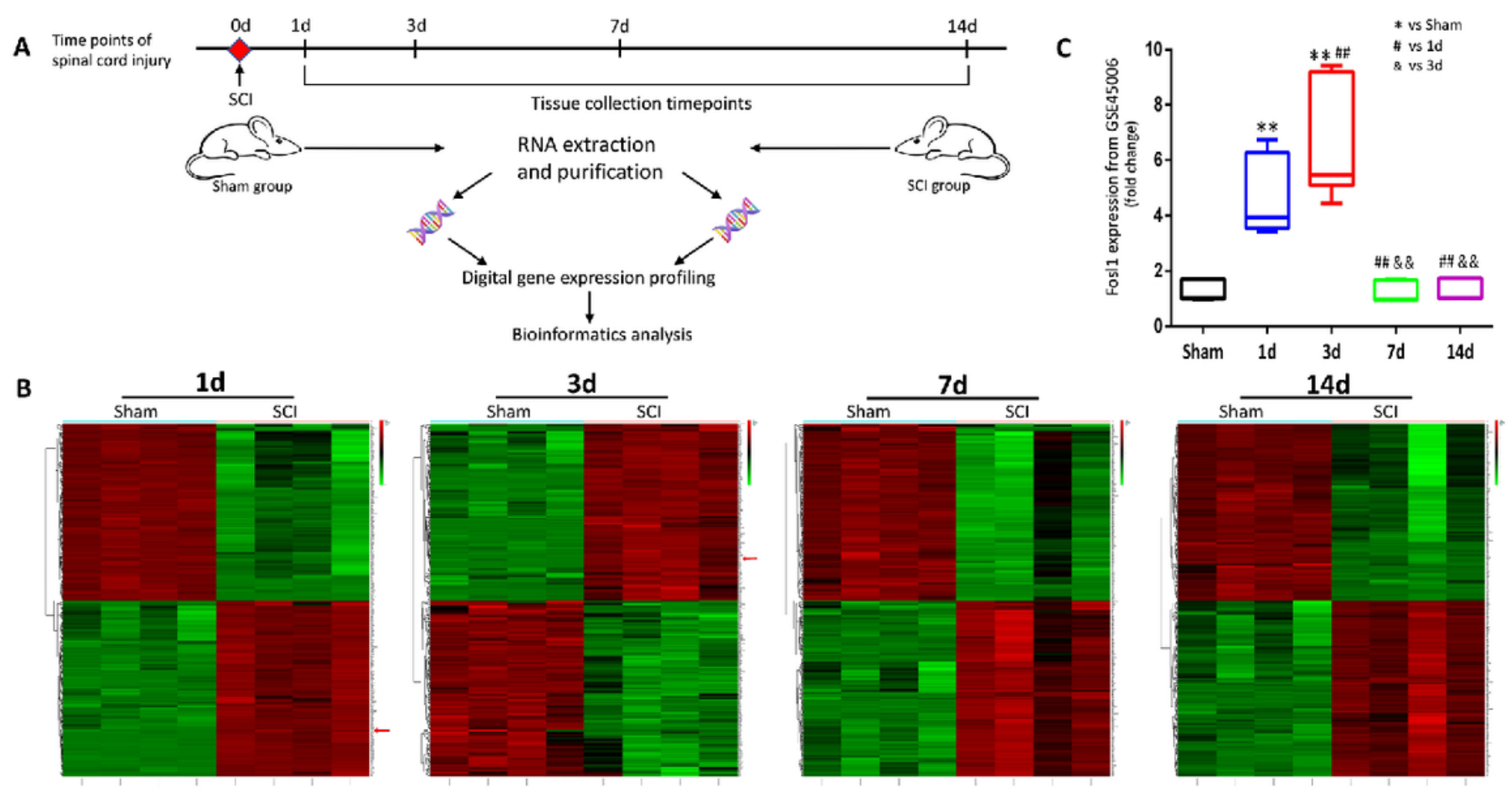

\section{Figure 1}

The mRNA of Fosl 1 was highly expressed in rats following $\mathrm{SCl}$ at $1 \mathrm{~d}$ and $3 \mathrm{~d}$ postoperation. A: Schematic of the experimental strategy used to screen the potential differential genes in the GEO database. B: The heatmaps generated by $\mathrm{R}$ language using the mRNA expression data of the $\mathrm{SCl}$ group and the sham group in GEO at $1 \mathrm{~d}, 3 \mathrm{~d}, 7 \mathrm{~d}$, and $14 \mathrm{~d}$ postoperation. C: The Fos 1 mRNA expression (normalized to that of the sham group) in the spinal cord of rats suffering from SCl at $1 d, 3 d, 7 d$, and 14 d postoperation $(n=4)$. All data are presented as the $M \pm S E M$. ${ }^{*} p<0.05$ vs. sham group, ${ }^{\star \star} p<0.01$ vs. sham group, $\# p<0.05$ vs. $S C l$ group at $1 \mathrm{~d}$ postoperation, \#\#p<0.01 vs. SCl group at $1 \mathrm{~d}$ postoperation, $\& p<0.05$ vs. $\mathrm{SCl}$ group at $3 \mathrm{~d}$ postoperation, $\& \& p<0.01 \mathrm{vs}$. $\mathrm{SCl}$ group at $3 \mathrm{~d}$ postoperation by ANOVA. 


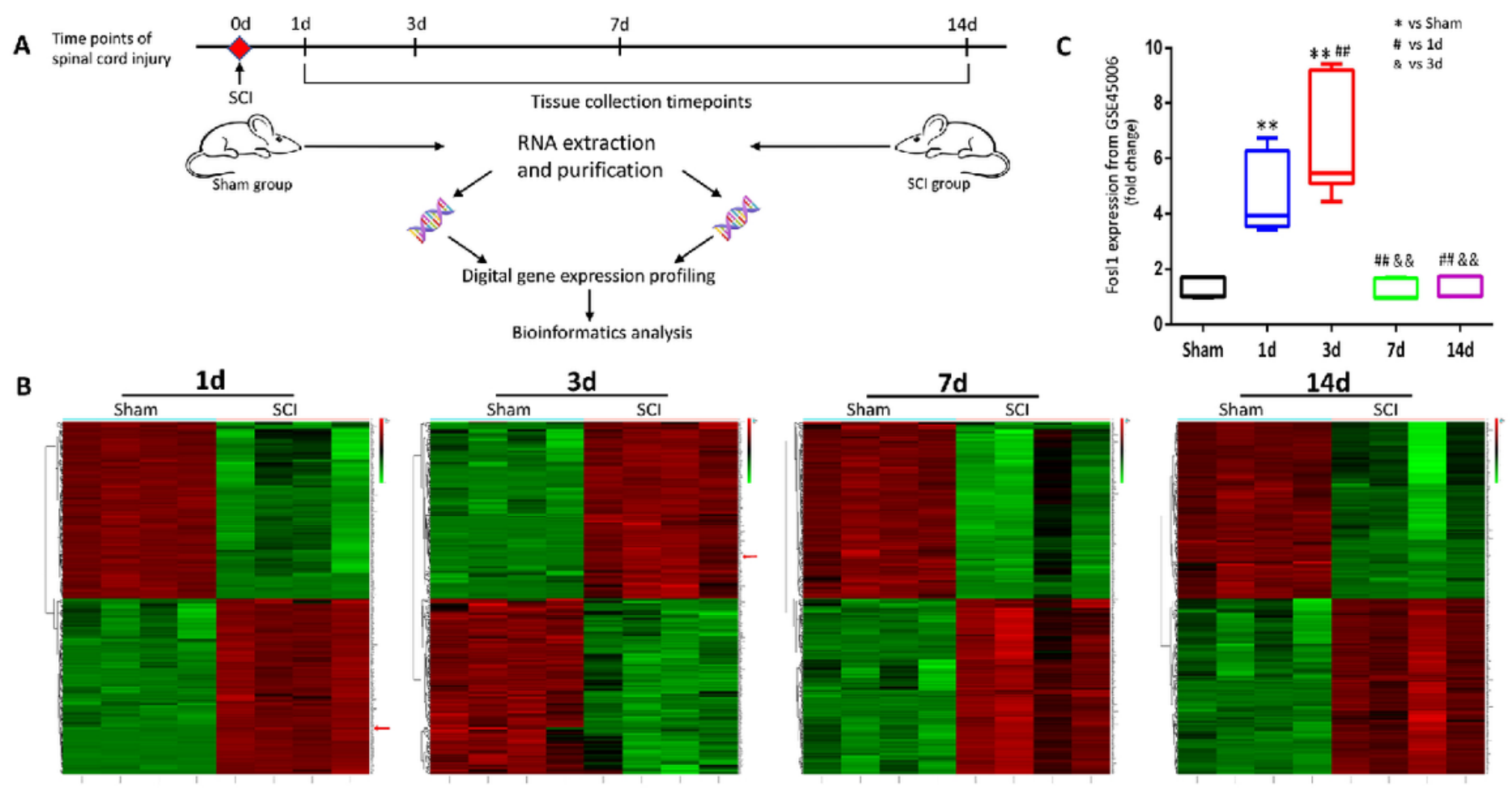

\section{Figure 1}

The mRNA of Fosl 1 was highly expressed in rats following $\mathrm{SCl}$ at $1 \mathrm{~d}$ and $3 \mathrm{~d}$ postoperation. A: Schematic of the experimental strategy used to screen the potential differential genes in the GEO database. B: The heatmaps generated by $\mathrm{R}$ language using the mRNA expression data of the $\mathrm{SCl}$ group and the sham group in GEO at $1 \mathrm{~d}, 3 \mathrm{~d}, 7 \mathrm{~d}$, and $14 \mathrm{~d}$ postoperation. C: The Fos 1 mRNA expression (normalized to that of the sham group) in the spinal cord of rats suffering from SCl at $1 \mathrm{~d}, 3 \mathrm{~d}, 7 \mathrm{~d}$, and 14 d postoperation $(n=4)$. All data are presented as the $M \pm S E M$. ${ }^{*} p<0.05$ vs. sham group, ${ }^{*} p<<0.01$ vs. sham group, $\# p<0.05$ vs. $S C l$ group at $1 \mathrm{~d}$ postoperation, \#\#p<0.01 vs. $S C l$ group at $1 \mathrm{~d}$ postoperation, \&p<0.05 vs. $\mathrm{SCl}$ group at $3 \mathrm{~d}$ postoperation, $\& \& p<0.01 \mathrm{vs}$. $\mathrm{SCl}$ group at $3 \mathrm{~d}$ postoperation by ANOVA. 


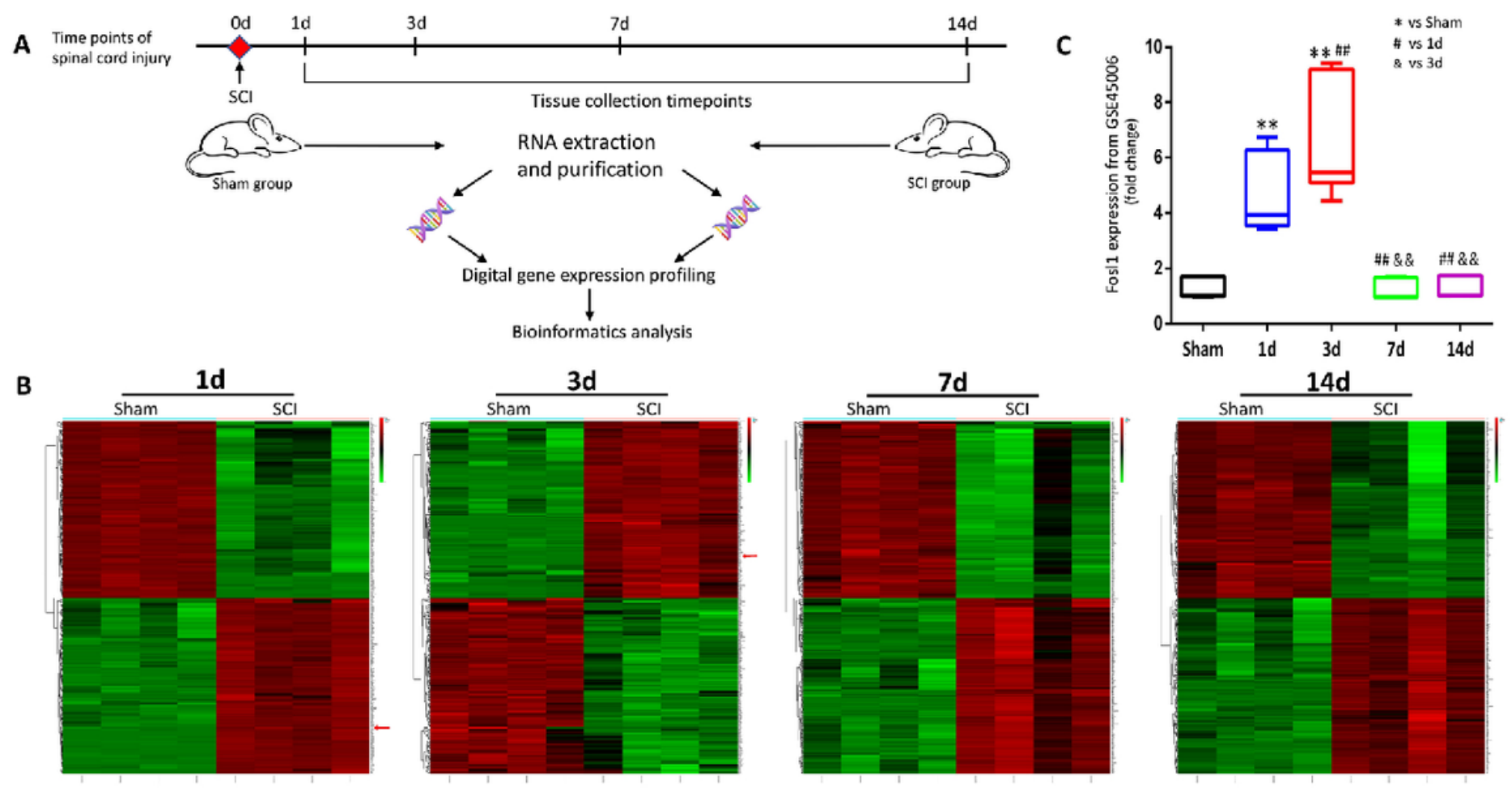

\section{Figure 1}

The mRNA of Fosl 1 was highly expressed in rats following $\mathrm{SCl}$ at $1 \mathrm{~d}$ and $3 \mathrm{~d}$ postoperation. A: Schematic of the experimental strategy used to screen the potential differential genes in the GEO database. B: The heatmaps generated by $\mathrm{R}$ language using the mRNA expression data of the $\mathrm{SCl}$ group and the sham group in GEO at $1 \mathrm{~d}, 3 \mathrm{~d}, 7 \mathrm{~d}$, and $14 \mathrm{~d}$ postoperation. C: The Fosl 1 mRNA expression (normalized to that of the sham group) in the spinal cord of rats suffering from SCl at $1 \mathrm{~d}, 3 \mathrm{~d}, 7 \mathrm{~d}$, and 14 d postoperation $(n=4)$. All data are presented as the $M \pm S E M$. ${ }^{*} p<0.05$ vs. sham group, ${ }^{*} p<<0.01$ vs. sham group, $\# p<0.05$ vs. $S C l$ group at $1 \mathrm{~d}$ postoperation, \#\#p<0.01 vs. $S C l$ group at $1 \mathrm{~d}$ postoperation, \&p<0.05 vs. $\mathrm{SCl}$ group at $3 \mathrm{~d}$ postoperation, $\& \& p<0.01 \mathrm{vs}$. $\mathrm{SCl}$ group at $3 \mathrm{~d}$ postoperation by ANOVA. 

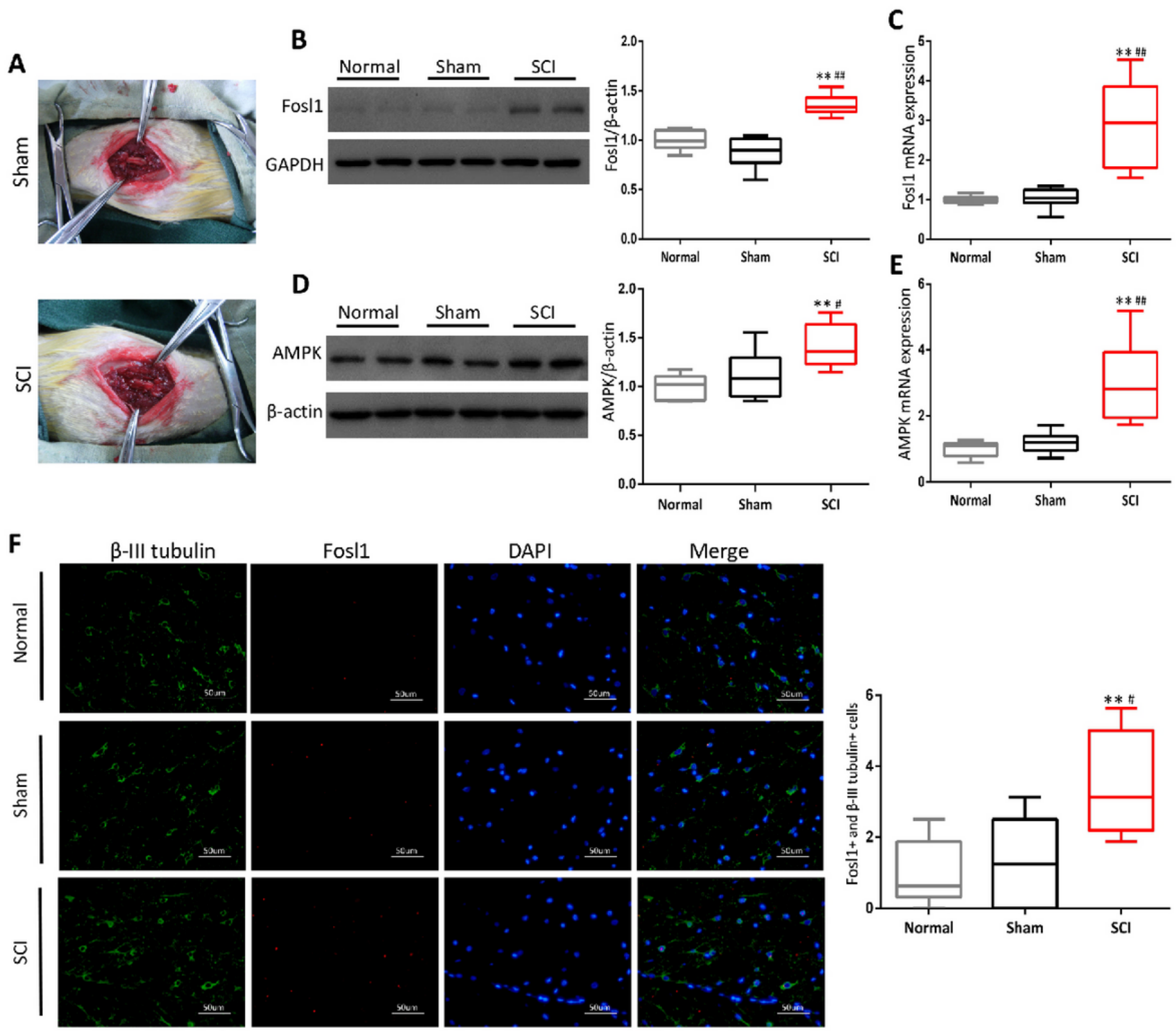

\section{Figure 2}

The mRNA and protein expression levels of Fosl1 and AMPK were increased significantly, and Fosl1 was highly expressed in neurons in rats following SCl. A: The model pictures of the rats in the sham group that received a laminectomy and the $\mathrm{SCl}$ group that suffered from a $\mathrm{SCl}$ surgery. B-C: The protein and mRNA expression levels of Fosl1 among the three groups were detected using western blotting and qRTPCR at $1 \mathrm{~d}$ postoperation. D-E: The protein and mRNA expression levels of AMPK among the three groups were detected using western blotting and qRT-PCR at $1 \mathrm{~d}$ postoperation. F: The Fosl 1 expression in neurons was examined using immunofluorescence double-label staining at $1 \mathrm{~d}$ postoperation (magnification $\times 400$ ). All data are presented as the $M \pm S E M\left(n=6\right.$ in each group). ${ }^{\star} p<0.05$ vs. normal group, ${ }^{* *} p<0.01$ vs. normal group, $\# p<0.05$ vs. sham group, $\# \# p<0.01$ vs. sham group by ANOVA. 

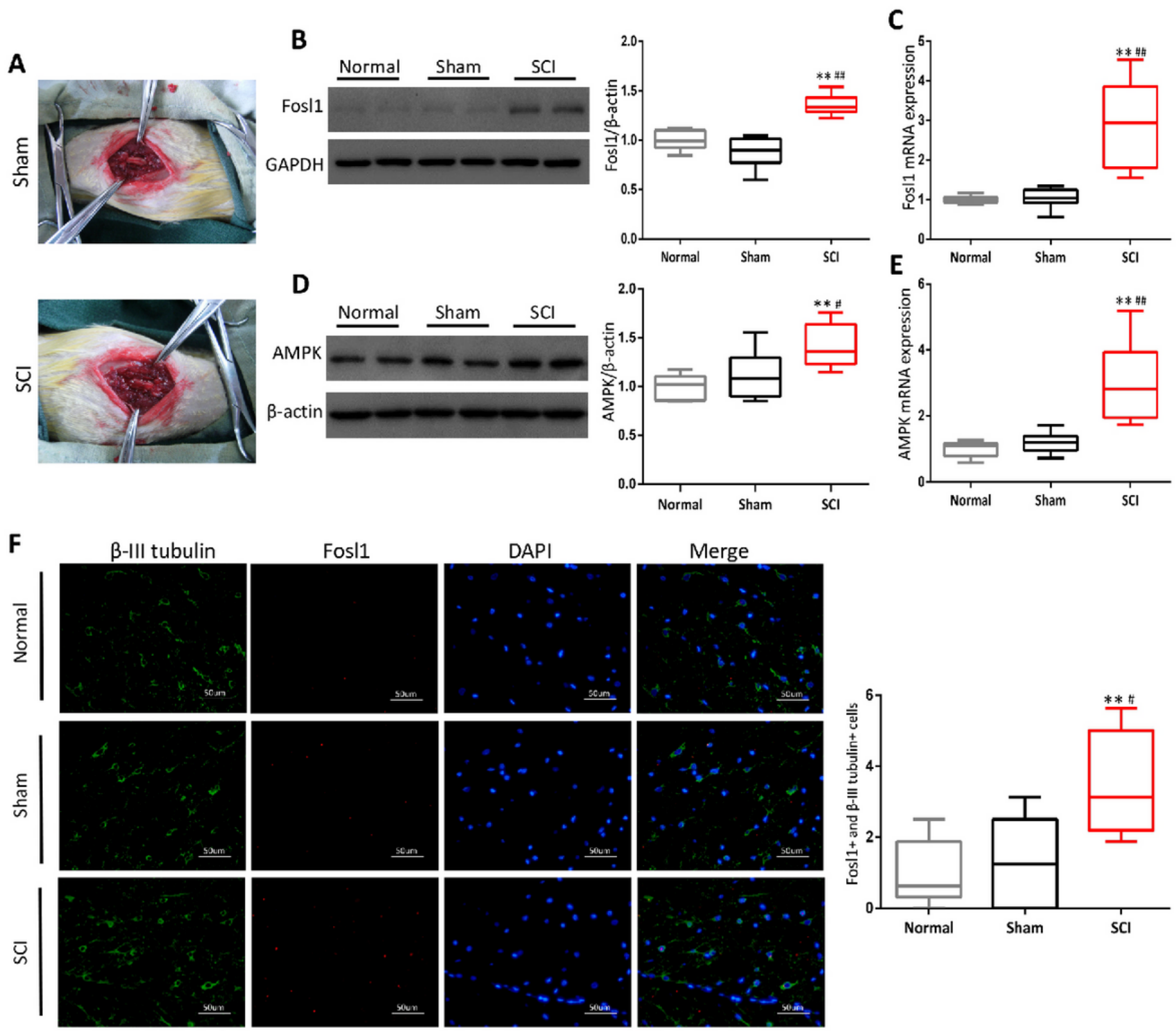

\section{Figure 2}

The mRNA and protein expression levels of Fosl1 and AMPK were increased significantly, and Fosl1 was highly expressed in neurons in rats following SCl. A: The model pictures of the rats in the sham group that received a laminectomy and the $\mathrm{SCl}$ group that suffered from a $\mathrm{SCl}$ surgery. B-C: The protein and mRNA expression levels of Fosl1 among the three groups were detected using western blotting and qRTPCR at $1 \mathrm{~d}$ postoperation. D-E: The protein and mRNA expression levels of AMPK among the three groups were detected using western blotting and qRT-PCR at $1 \mathrm{~d}$ postoperation. F: The Fosl 1 expression in neurons was examined using immunofluorescence double-label staining at $1 \mathrm{~d}$ postoperation (magnification $\times 400$ ). All data are presented as the $M \pm S E M\left(n=6\right.$ in each group). ${ }^{\star} p<0.05$ vs. normal group, ${ }^{* *} p<0.01$ vs. normal group, $\# p<0.05$ vs. sham group, $\# \# p<0.01$ vs. sham group by ANOVA. 

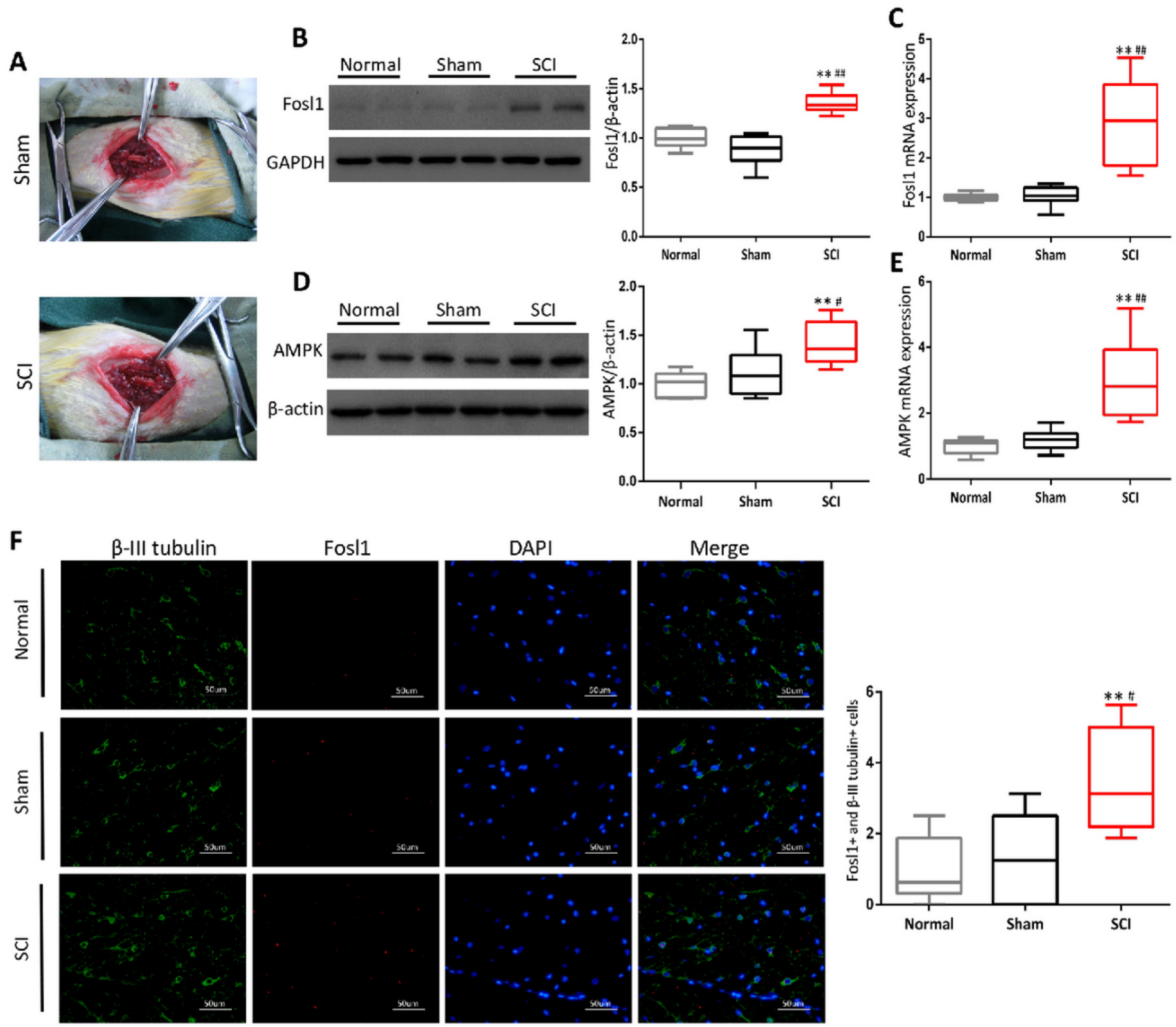

\section{Figure 2}

The mRNA and protein expression levels of Fosl1 and AMPK were increased significantly, and Fosl1 was highly expressed in neurons in rats following SCl. A: The model pictures of the rats in the sham group that received a laminectomy and the $\mathrm{SCl}$ group that suffered from a $\mathrm{SCl}$ surgery. B-C: The protein and mRNA expression levels of Fosl1 among the three groups were detected using western blotting and qRTPCR at $1 \mathrm{~d}$ postoperation. D-E: The protein and mRNA expression levels of AMPK among the three groups were detected using western blotting and qRT-PCR at $1 \mathrm{~d}$ postoperation. F: The Fosl 1 expression in neurons was examined using immunofluorescence double-label staining at $1 \mathrm{~d}$ postoperation (magnification $\times 400$ ). All data are presented as the $M \pm S E M\left(n=6\right.$ in each group). ${ }^{*} p<0.05$ vs. normal group, ${ }^{* *} p<0.01$ vs. normal group, $\# p<0.05$ vs. sham group, $\# \# p<0.01$ vs. sham group by ANOVA. 

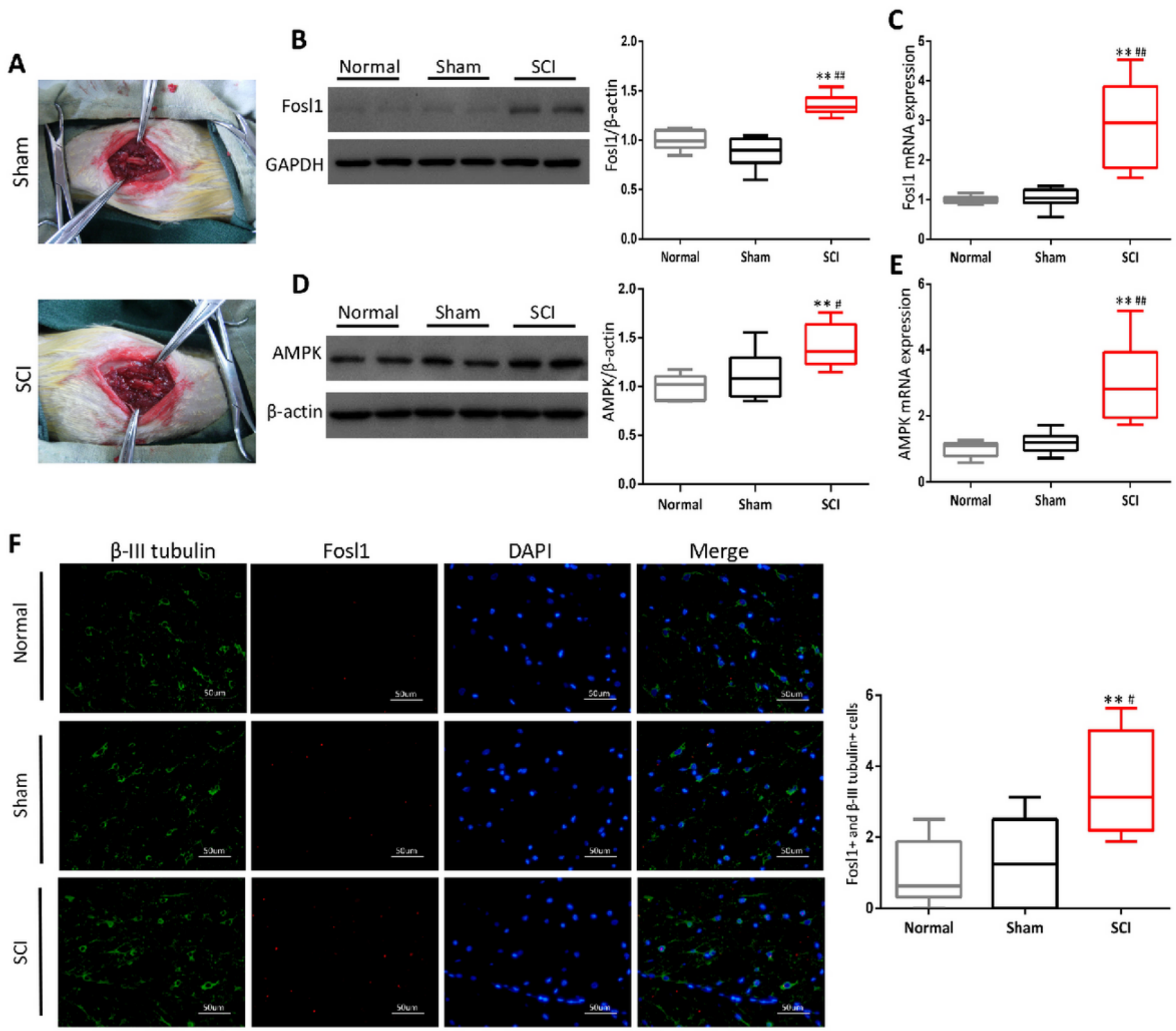

\section{Figure 2}

The mRNA and protein expression levels of Fosl1 and AMPK were increased significantly, and Fosl1 was highly expressed in neurons in rats following SCl. A: The model pictures of the rats in the sham group that received a laminectomy and the $\mathrm{SCl}$ group that suffered from a $\mathrm{SCl}$ surgery. B-C: The protein and mRNA expression levels of Fosl1 among the three groups were detected using western blotting and qRTPCR at $1 \mathrm{~d}$ postoperation. D-E: The protein and mRNA expression levels of AMPK among the three groups were detected using western blotting and qRT-PCR at $1 \mathrm{~d}$ postoperation. F: The Fosl 1 expression in neurons was examined using immunofluorescence double-label staining at $1 \mathrm{~d}$ postoperation (magnification $\times 400$ ). All data are presented as the $M \pm S E M\left(n=6\right.$ in each group). ${ }^{\star} p<0.05$ vs. normal group, ${ }^{* *} p<0.01$ vs. normal group, $\# p<0.05$ vs. sham group, $\# \# p<0.01$ vs. sham group by ANOVA. 

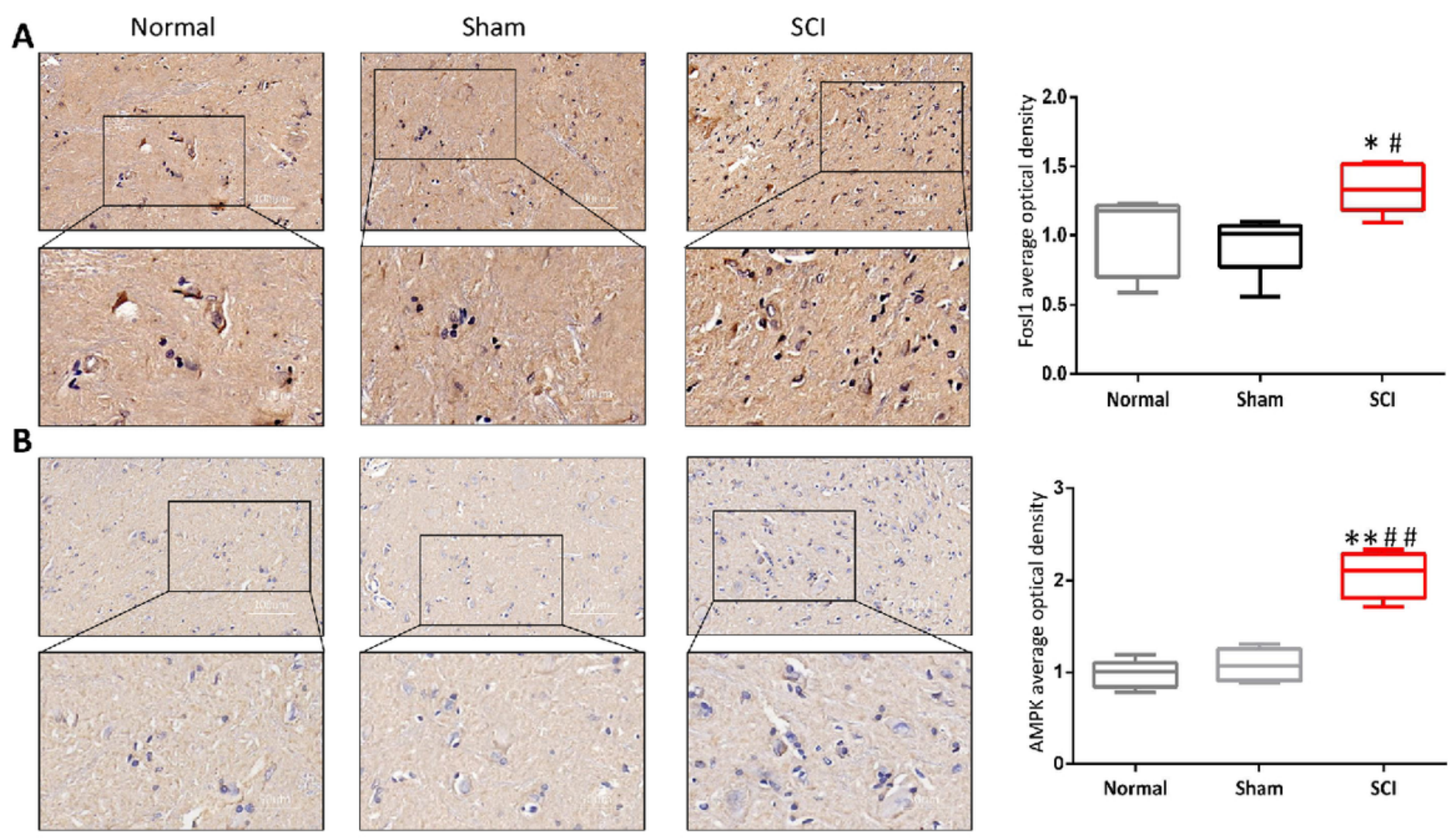

\section{Figure 3}

The Fosl1 and AMPK was enhanced significantly at histologic level following SCl. A: The Fosl1 expression in spinal cord was checked using immunohistochemical staining at $1 \mathrm{~d}$ postoperation (magnification $\times 200, \times 400$ ). B: The AMPK expression in spinal cord was detected using immunohistochemical staining at $1 \mathrm{~d}$ postoperation (magnification $\times 200, \times 400$ ). All data are presented as the $M \pm \operatorname{SEM}\left(n=6\right.$ in each group). ${ }^{\star} p<0.05$ vs. normal group, ${ }^{\star *} p<0.01$ vs. normal group, $\# p<0.05$ vs. sham group, \#\#p<0.01 vs. sham group. 

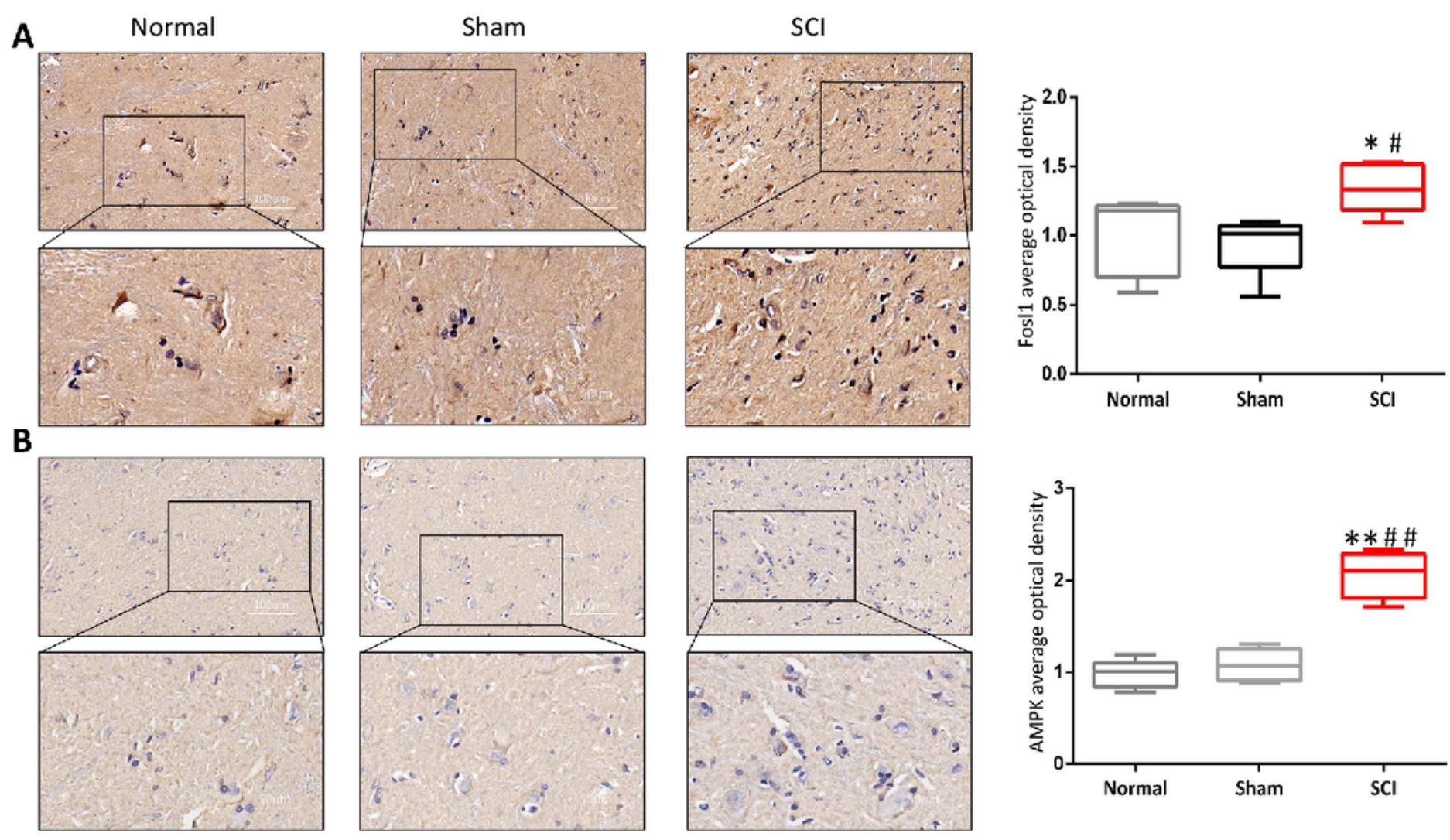

\section{Figure 3}

The Fosl1 and AMPK was enhanced significantly at histologic level following SCl. A: The Fosl1 expression in spinal cord was checked using immunohistochemical staining at $1 \mathrm{~d}$ postoperation (magnification $\times 200, \times 400$ ). B: The AMPK expression in spinal cord was detected using immunohistochemical staining at $1 \mathrm{~d}$ postoperation (magnification $\times 200, \times 400$ ). All data are presented as the $M \pm \operatorname{SEM}\left(n=6\right.$ in each group). ${ }^{\star} p<0.05$ vs. normal group, ${ }^{\star *} p<0.01$ vs. normal group, $\# p<0.05$ vs. sham group, \#\#p<0.01 vs. sham group. 

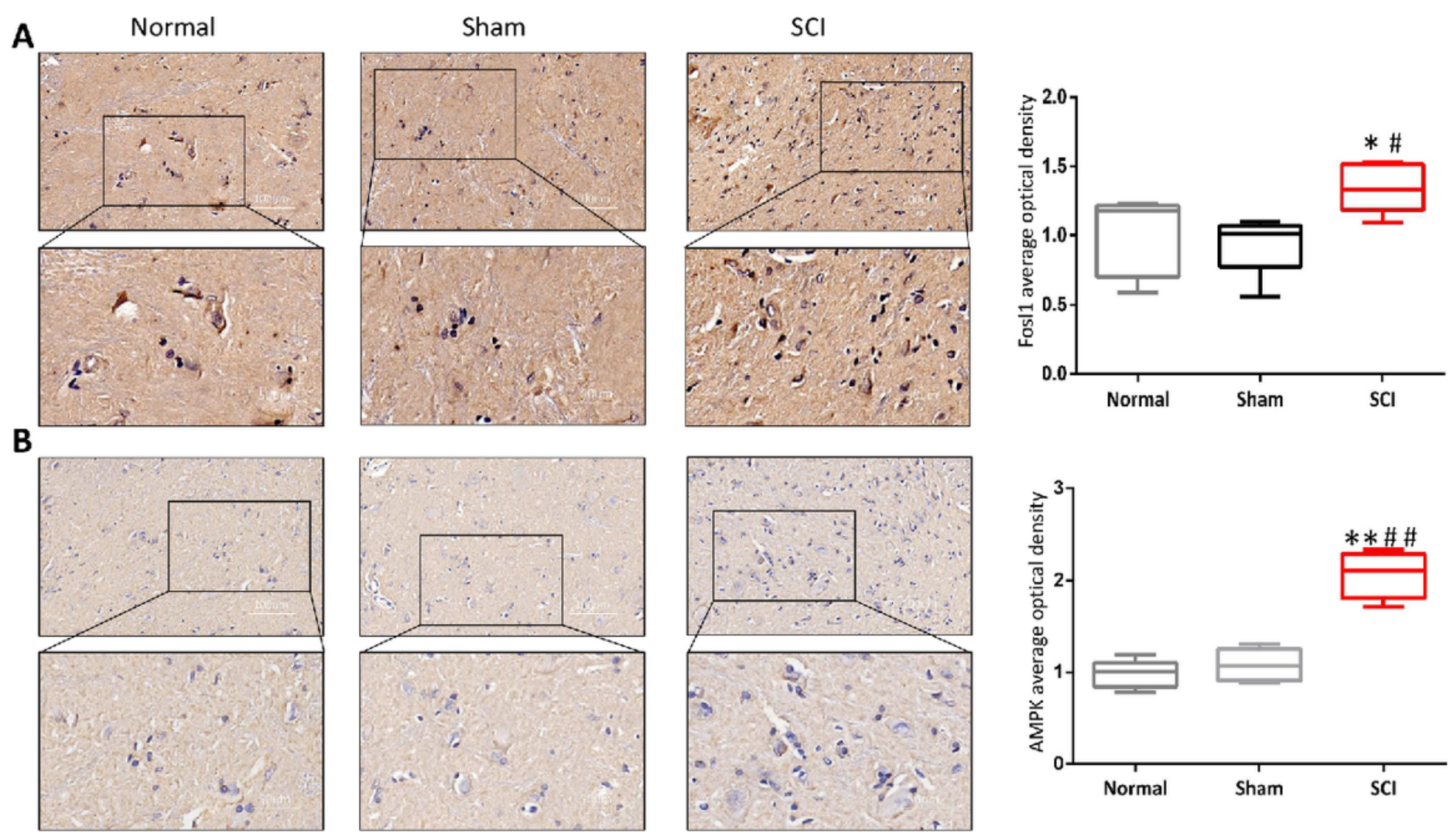

\section{Figure 3}

The Fosl1 and AMPK was enhanced significantly at histologic level following SCl. A: The Fosl1 expression in spinal cord was checked using immunohistochemical staining at $1 \mathrm{~d}$ postoperation (magnification $\times 200, \times 400$ ). B: The AMPK expression in spinal cord was detected using immunohistochemical staining at $1 \mathrm{~d}$ postoperation (magnification $\times 200, \times 400$ ). All data are presented as the $M \pm \operatorname{SEM}\left(n=6\right.$ in each group). ${ }^{\star} p<0.05$ vs. normal group, ${ }^{\star *} p<0.01$ vs. normal group, $\# p<0.05$ vs. sham group, \#\#p<0.01 vs. sham group. 

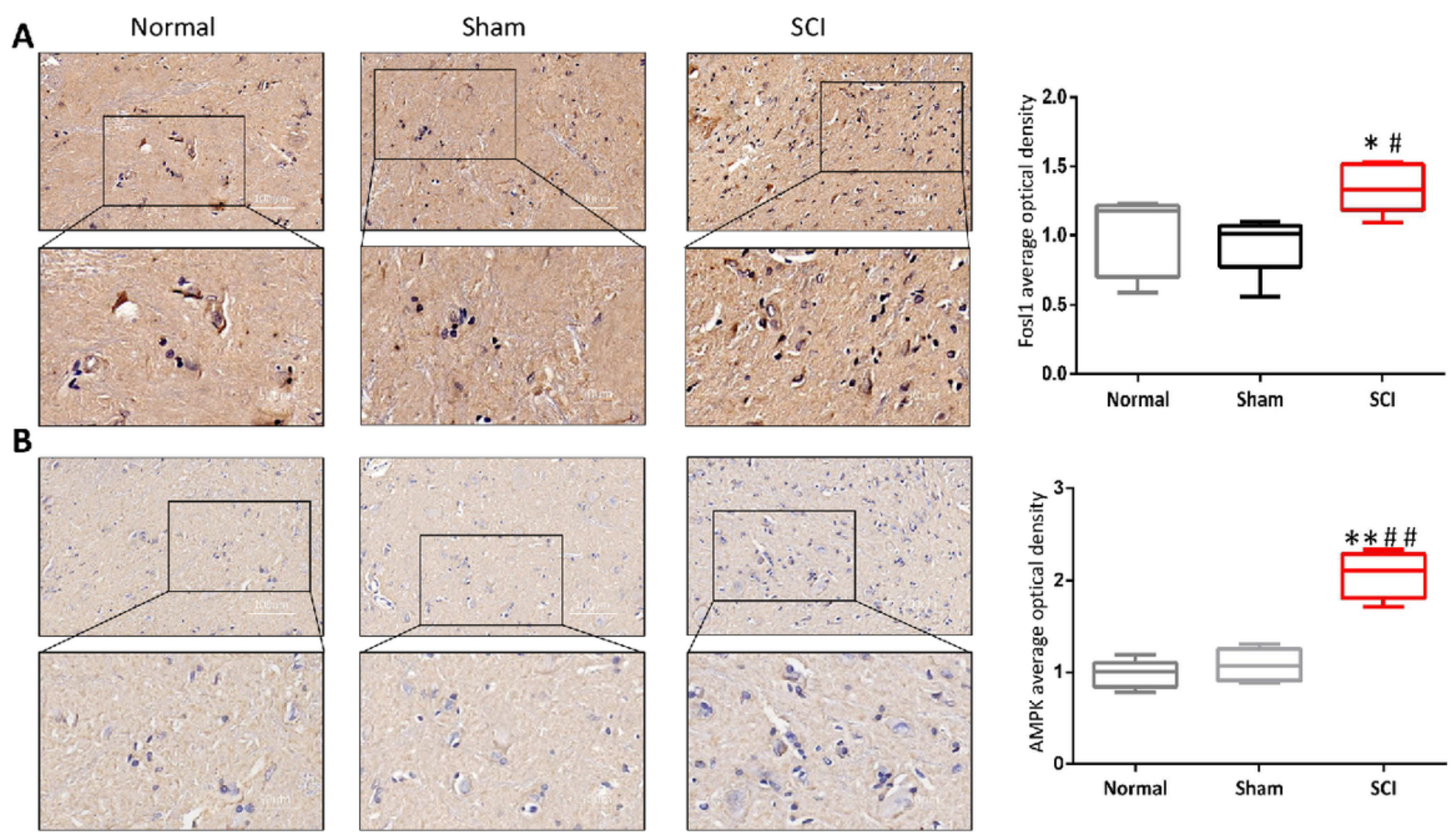

\section{Figure 3}

The Fosl1 and AMPK was enhanced significantly at histologic level following SCl. A: The Fosl1 expression in spinal cord was checked using immunohistochemical staining at $1 \mathrm{~d}$ postoperation (magnification $\times 200, \times 400$ ). B: The AMPK expression in spinal cord was detected using immunohistochemical staining at $1 \mathrm{~d}$ postoperation (magnification $\times 200, \times 400$ ). All data are presented as the $M \pm \operatorname{SEM}\left(n=6\right.$ in each group). ${ }^{\star} p<0.05$ vs. normal group, ${ }^{\star *} p<0.01$ vs. normal group, $\# p<0.05$ vs. sham group, \#\#p<0.01 vs. sham group. 

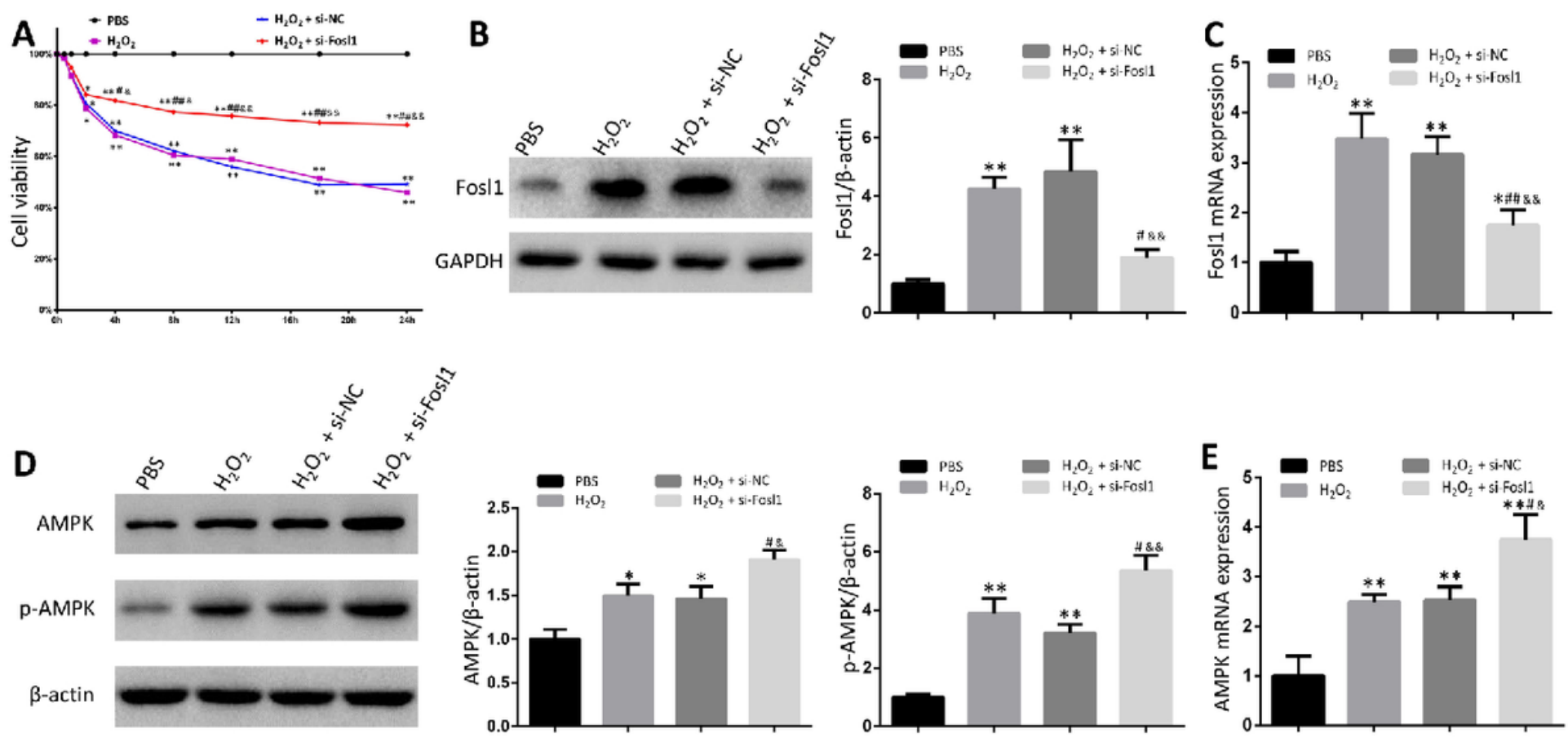

\section{Figure 4}

The Fosl 1 and AMPK were highly expressed in PC-12 cells with H2O2-induced injury at $24 \mathrm{~h}$. si-Fosl1 downregulated Fos 11 expression and enhanced AMPK at the protein and mRNA levels at $24 \mathrm{~h}$ and increased the phosphorylation of AMPK and cell viability. A: Cell viability of the four groups following treatment for $48 \mathrm{~h}$. B-C: The mRNA and protein expression levels of Fosl1 were examined using western blotting and qRT-PCR in the four groups. D-E: The mRNA and protein expression levels of AMPK were examined using western blotting and qRT-PCR in the four groups, and p-AMPK expression was detected by western blotting. All data are presented as the $M \pm S E M\left(n=3\right.$ in each group). ${ }^{*} p<0.05$ vs. PBS group, ${ }^{* *} p<0.01$ vs. PBS group, $\# p<0.05$ vs. $\mathrm{H} 2 \mathrm{O} 2$ group, $\# \# p<0.01$ vs. $\mathrm{H} 2 \mathrm{O} 2$ group, $\& \mathrm{p}<0.05$ vs. $\mathrm{H} 2 \mathrm{O} 2+$ si-NC group, \&\&p<0.01 vs. $\mathrm{H} 2 \mathrm{O} 2+$ si-NC group by ANOVA. 

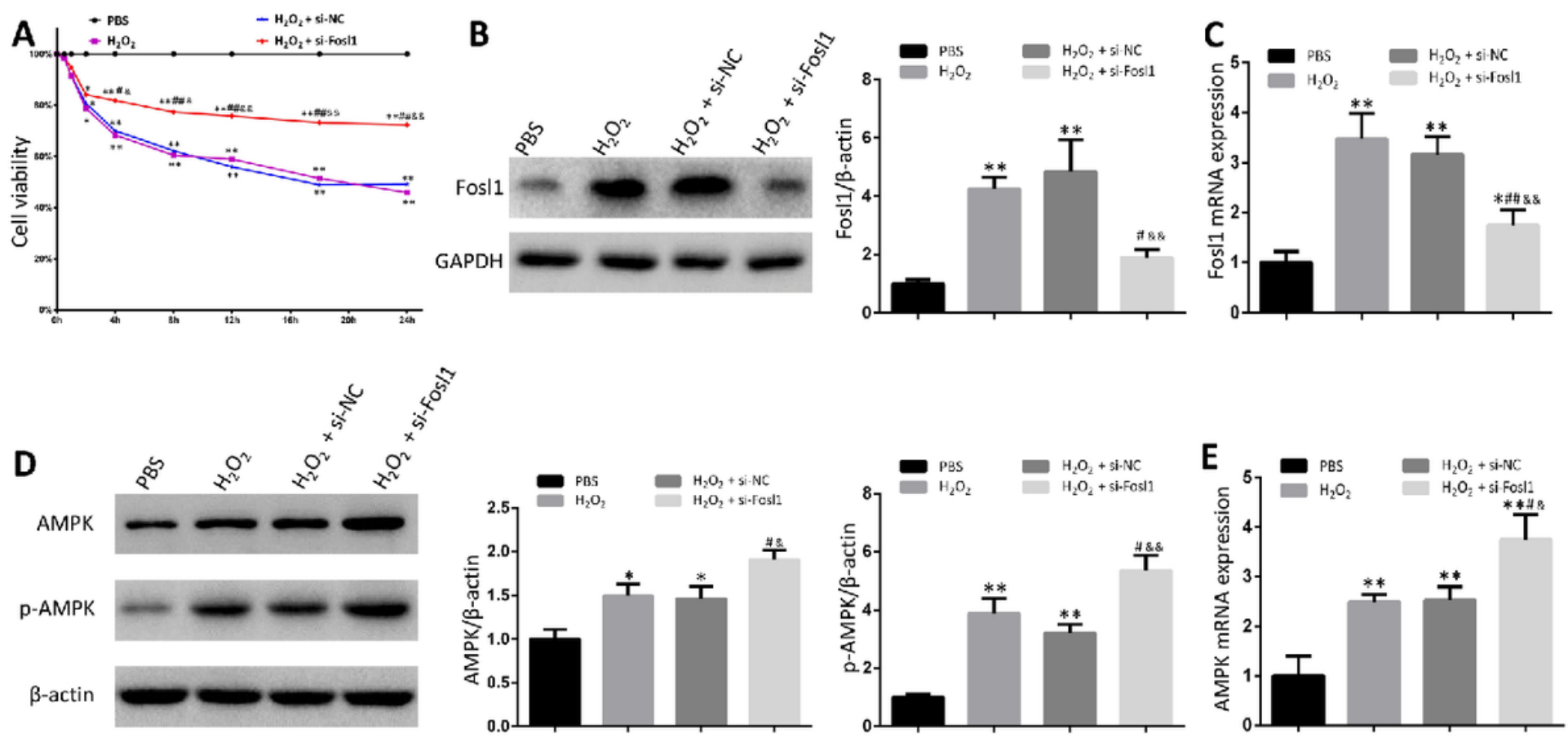

\section{Figure 4}

The Fosl 1 and AMPK were highly expressed in PC-12 cells with H2O2-induced injury at $24 \mathrm{~h}$. si-Fosl1 downregulated Fos 11 expression and enhanced AMPK at the protein and mRNA levels at $24 \mathrm{~h}$ and increased the phosphorylation of AMPK and cell viability. A: Cell viability of the four groups following treatment for $48 \mathrm{~h}$. B-C: The mRNA and protein expression levels of Fosl1 were examined using western blotting and qRT-PCR in the four groups. D-E: The mRNA and protein expression levels of AMPK were examined using western blotting and qRT-PCR in the four groups, and p-AMPK expression was detected by western blotting. All data are presented as the $M \pm S E M\left(n=3\right.$ in each group). ${ }^{*} p<0.05$ vs. PBS group, ${ }^{* *} p<0.01$ vs. PBS group, $\# p<0.05$ vs. $\mathrm{H} 2 \mathrm{O} 2$ group, $\# \# p<0.01$ vs. $\mathrm{H} 2 \mathrm{O} 2$ group, $\& \mathrm{p}<0.05$ vs. $\mathrm{H} 2 \mathrm{O} 2+$ si-NC group, \&\&p<0.01 vs. $\mathrm{H} 2 \mathrm{O} 2+$ si-NC group by ANOVA. 

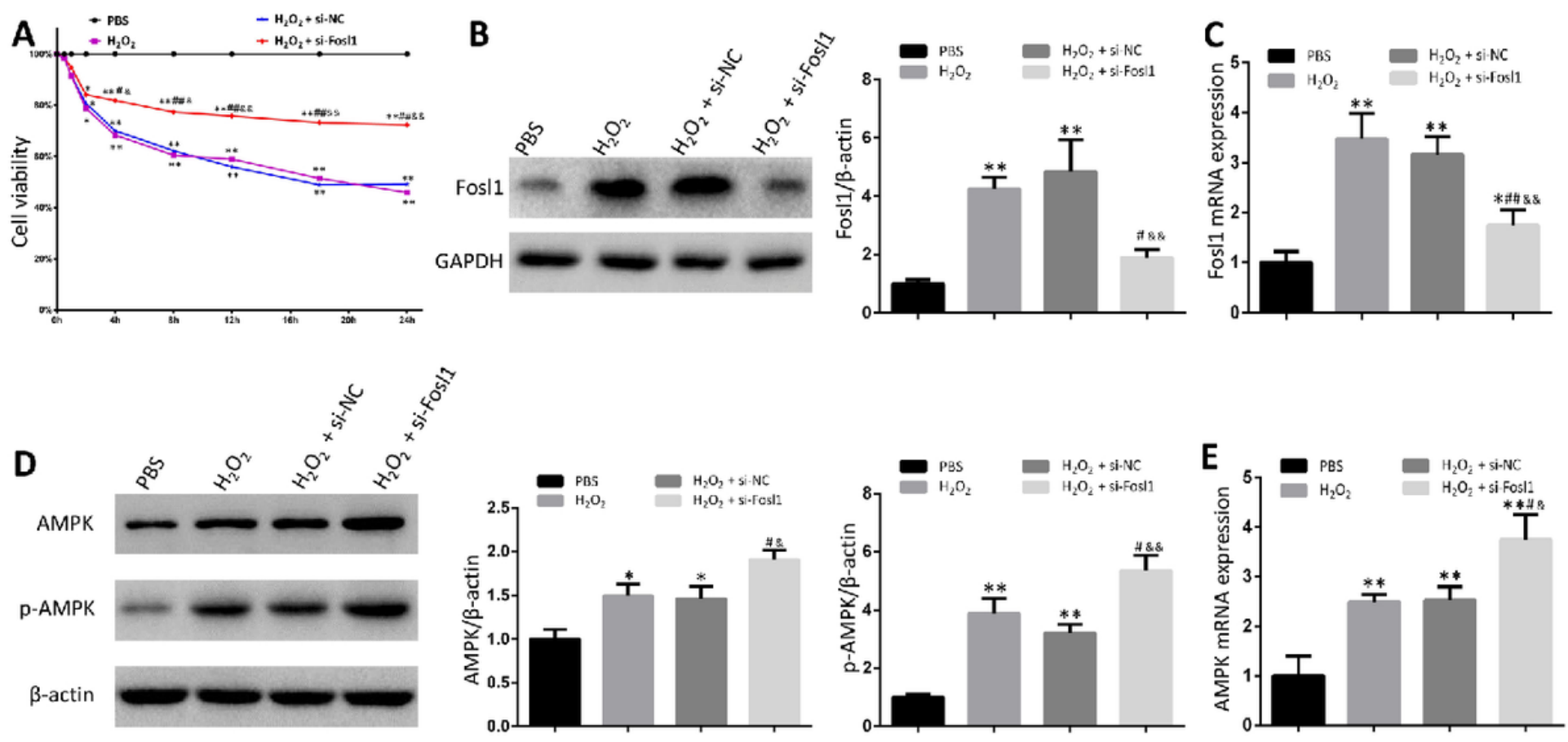

\section{Figure 4}

The Fosl 1 and AMPK were highly expressed in PC-12 cells with H2O2-induced injury at $24 \mathrm{~h}$. si-Fosl1 downregulated Fos 11 expression and enhanced AMPK at the protein and mRNA levels at $24 \mathrm{~h}$ and increased the phosphorylation of AMPK and cell viability. A: Cell viability of the four groups following treatment for $48 \mathrm{~h}$. B-C: The mRNA and protein expression levels of Fosl1 were examined using western blotting and qRT-PCR in the four groups. D-E: The mRNA and protein expression levels of AMPK were examined using western blotting and qRT-PCR in the four groups, and p-AMPK expression was detected by western blotting. All data are presented as the $M \pm S E M\left(n=3\right.$ in each group). ${ }^{*} p<0.05$ vs. PBS group, ${ }^{* *} p<0.01$ vs. PBS group, $\# p<0.05$ vs. $\mathrm{H} 2 \mathrm{O} 2$ group, $\# \# p<0.01$ vs. $\mathrm{H} 2 \mathrm{O} 2$ group, $\& \mathrm{p}<0.05$ vs. $\mathrm{H} 2 \mathrm{O} 2+$ si-NC group, \&\&p<0.01 vs. $\mathrm{H} 2 \mathrm{O} 2+$ si-NC group by ANOVA. 

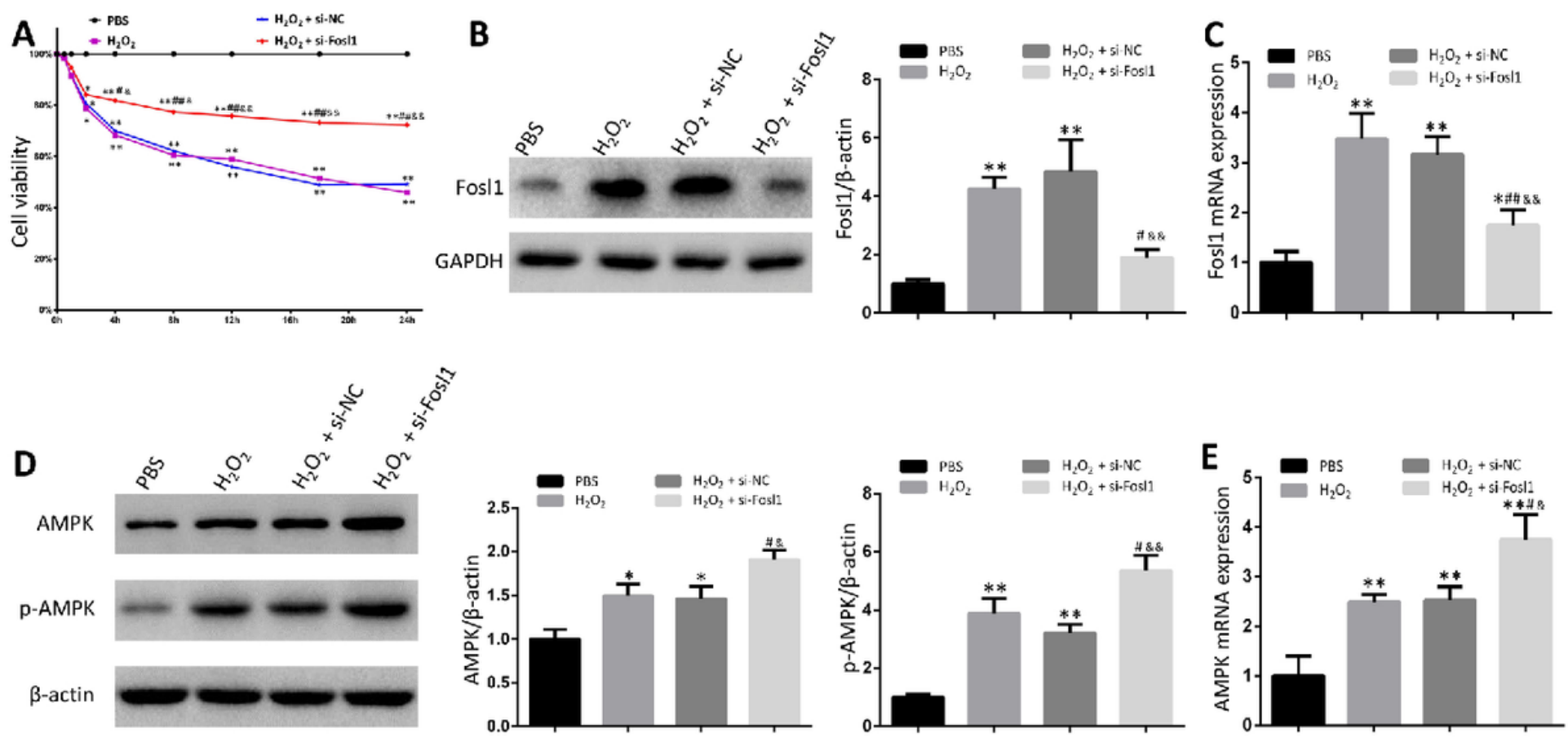

\section{Figure 4}

The Fosl 1 and AMPK were highly expressed in PC-12 cells with H2O2-induced injury at $24 \mathrm{~h}$. si-Fosl1 downregulated Fos 11 expression and enhanced AMPK at the protein and mRNA levels at $24 \mathrm{~h}$ and increased the phosphorylation of AMPK and cell viability. A: Cell viability of the four groups following treatment for $48 \mathrm{~h}$. B-C: The mRNA and protein expression levels of Fosl1 were examined using western blotting and qRT-PCR in the four groups. D-E: The mRNA and protein expression levels of AMPK were examined using western blotting and qRT-PCR in the four groups, and p-AMPK expression was detected by western blotting. All data are presented as the $M \pm S E M\left(n=3\right.$ in each group). ${ }^{*} p<0.05$ vs. PBS group, ${ }^{* *} p<0.01$ vs. PBS group, $\# p<0.05$ vs. $\mathrm{H} 2 \mathrm{O} 2$ group, $\# \# p<0.01$ vs. $\mathrm{H} 2 \mathrm{O} 2$ group, $\& \mathrm{p}<0.05$ vs. $\mathrm{H} 2 \mathrm{O} 2+$ si-NC group, \&\&p<0.01 vs. $\mathrm{H} 2 \mathrm{O} 2+$ si-NC group by ANOVA. 


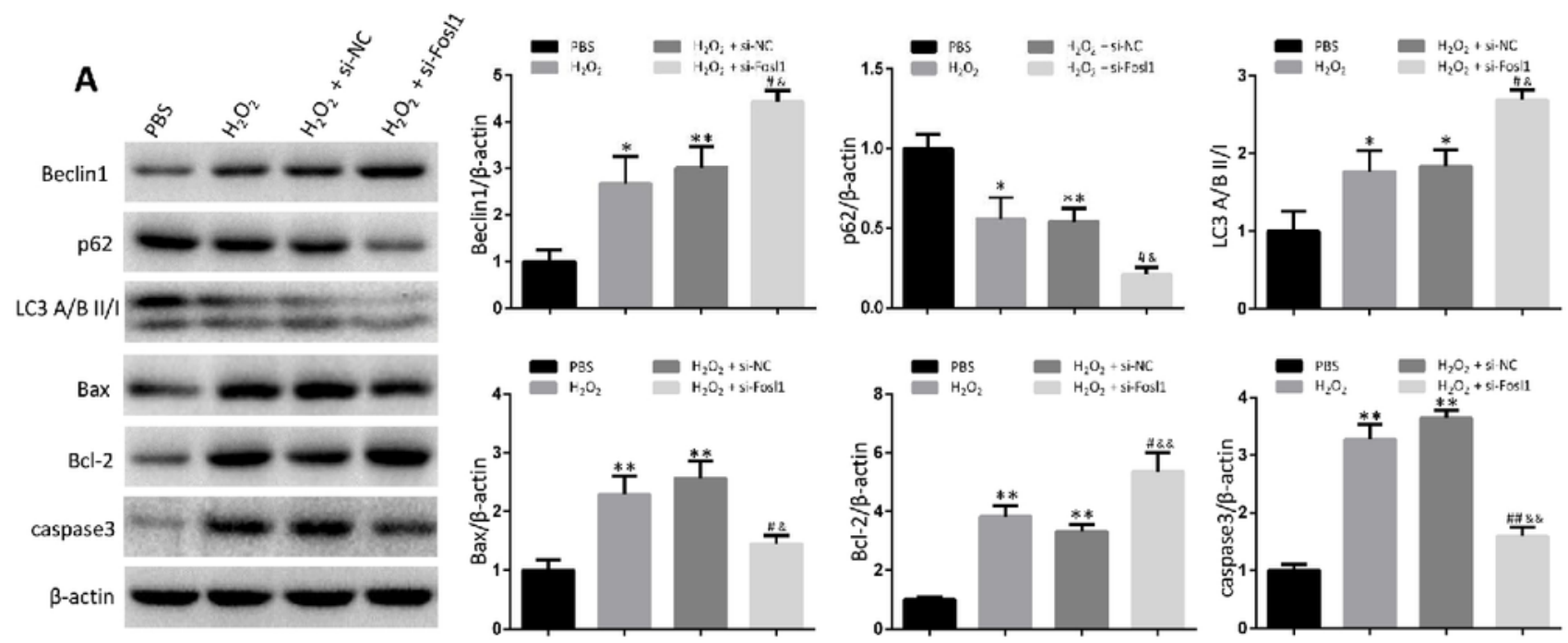

B
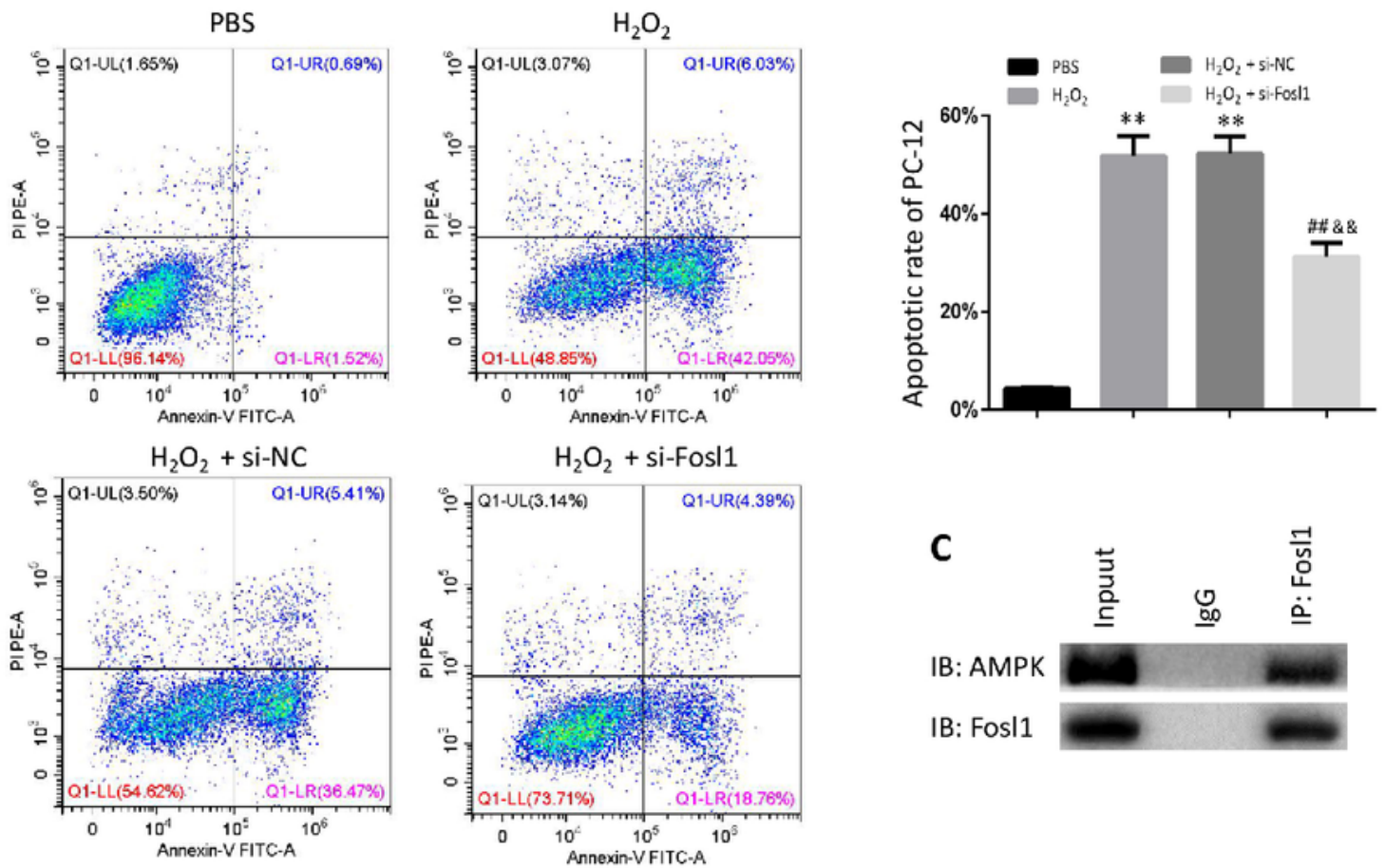

IB: AMPK

IB: Fosl1
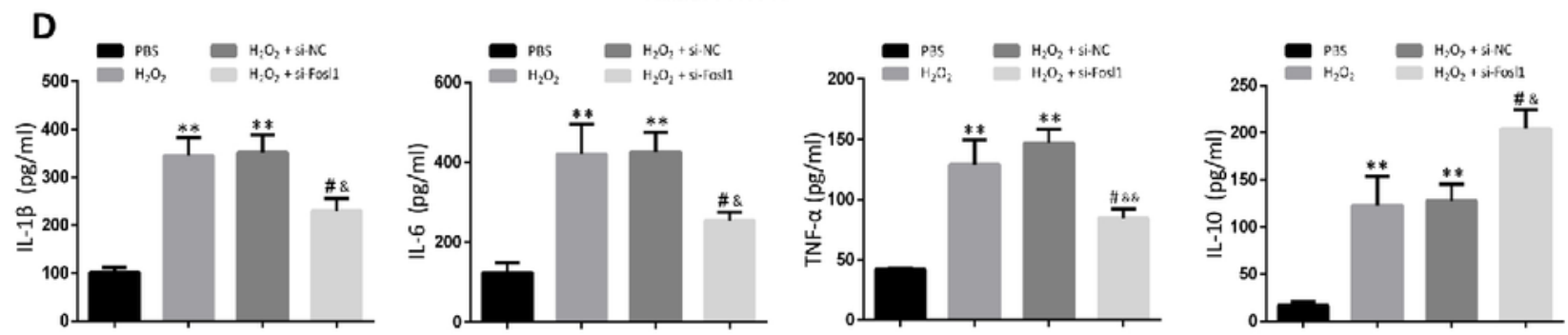

\section{Figure 5}

si-Fosl1 enhanced the autophagic activity and decreased the apoptosis and inflammation. A: The autophagic marker proteins (Beclin1, p62, LC3) and the apoptotic proteins (Bax, Bcl-2, caspase3) were detected using western blotting. B: The apoptotic cell rate was monitored by flow cytometry. C: The COIP was used to examine the interaction of Fosl 1 and AMPK in injured PC-12 cells. D: The inflammatory proteins (IL-1 $\beta$, IL-6, TNF-a, IL-10) were examined using ELISA. All data are presented as the M \pm SEM 
( $n=3$ in each group). ${ }^{\star} p<0.05$ vs. PBS group, ${ }^{\star *} p<0.01$ vs. PBS group, $\# p<0.05$ vs. H202 group, \#\#p<0.01 vs. H2O2 group, \&p<0.05 vs. H2O2 + si-NC group, \&\&p<0.01 vs. H2O2 + si-NC group by ANOVA.
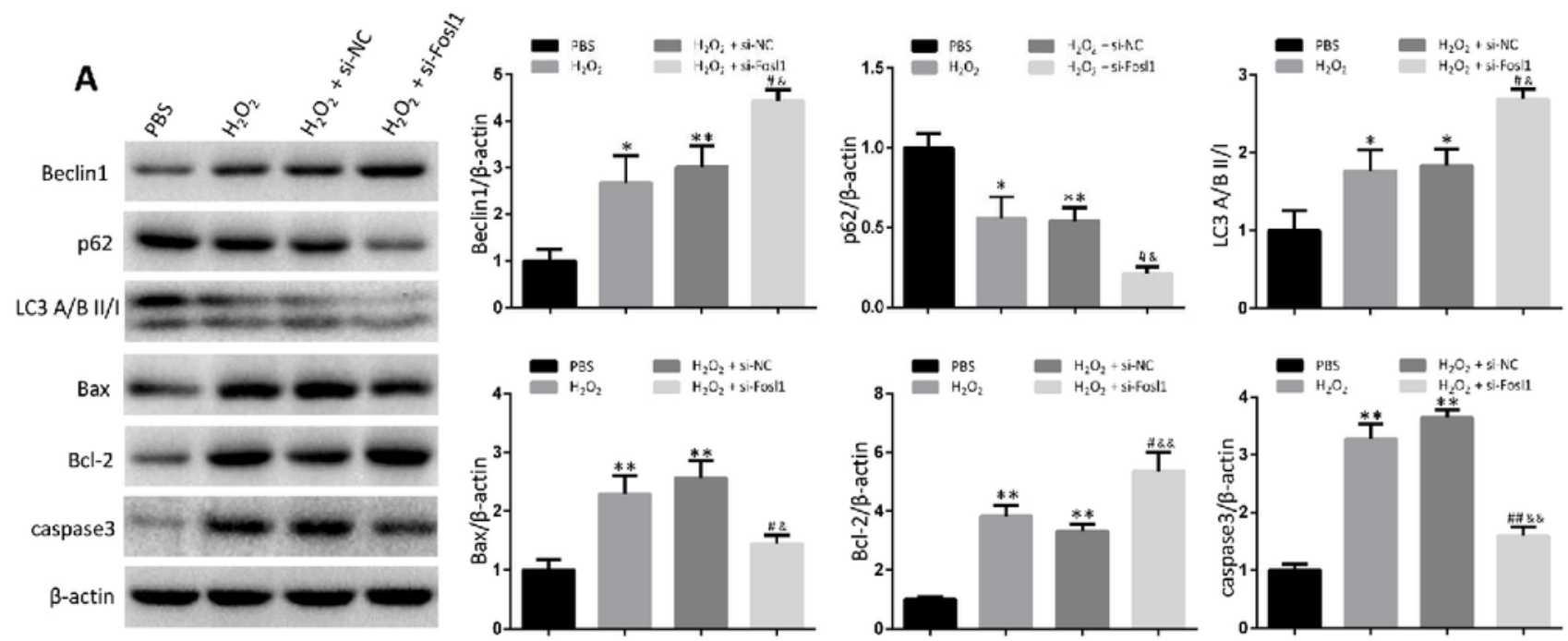

B
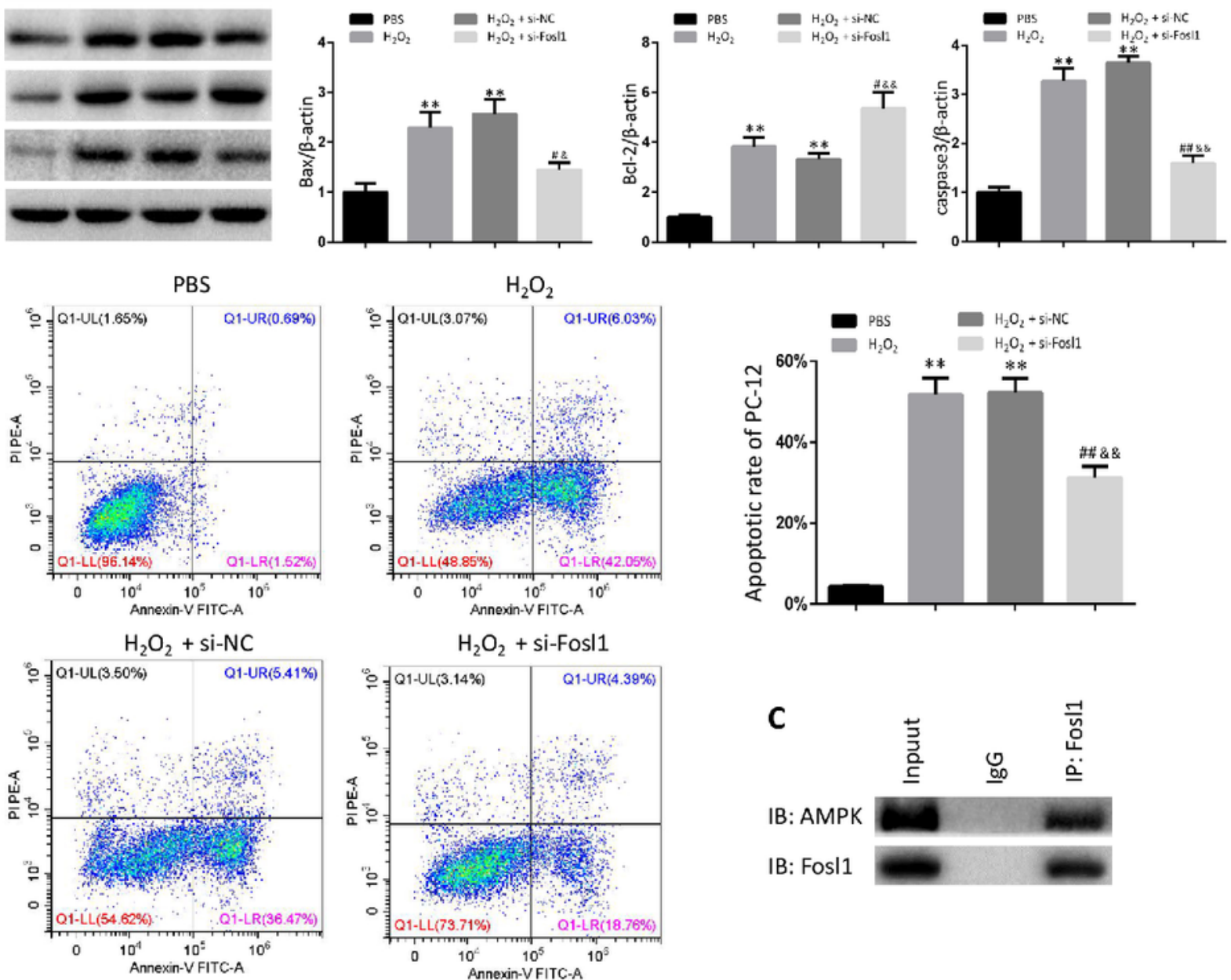

IB: AMPK

IB: Fosl1
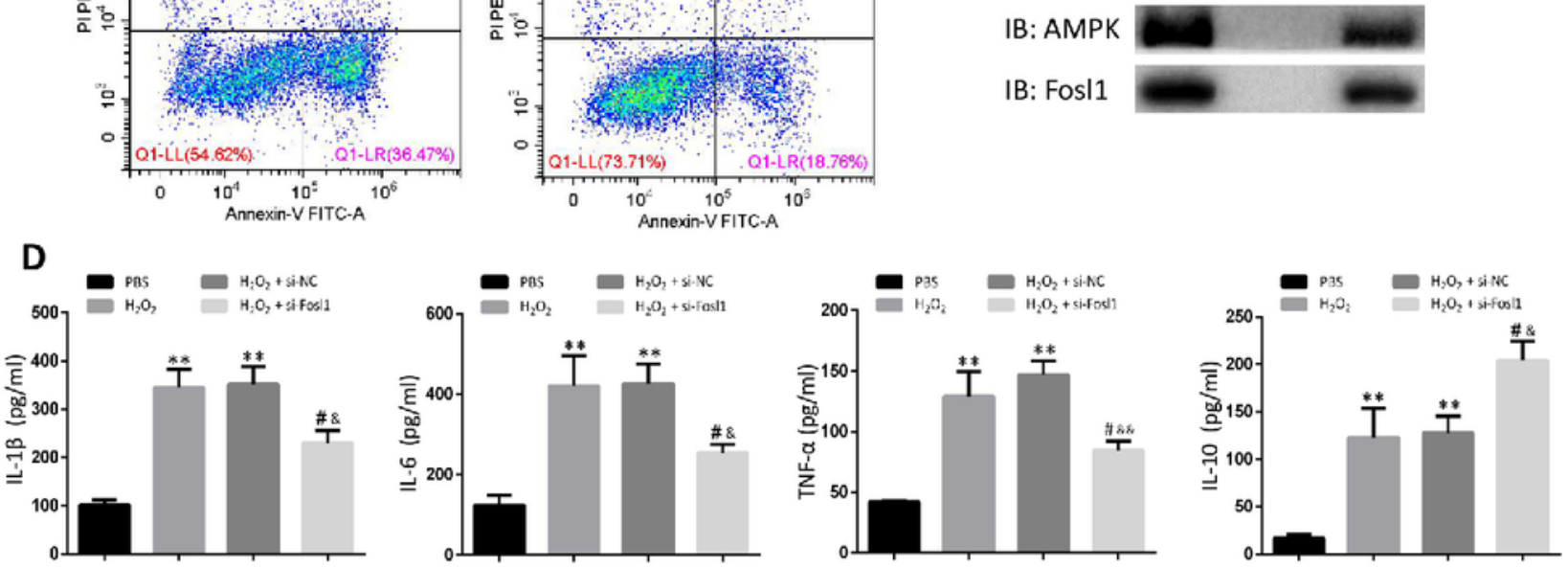

Figure 5

si-Fosl1 enhanced the autophagic activity and decreased the apoptosis and inflammation. A: The autophagic marker proteins (Beclin1, p62, LC3) and the apoptotic proteins (Bax, Bcl-2, caspase3) were detected using western blotting. B: The apoptotic cell rate was monitored by flow cytometry. C: The COIP 
was used to examine the interaction of Fosl 1 and AMPK in injured PC-12 cells. D: The inflammatory proteins (IL-1 $\beta, I L-6, T N F-a, I L-10)$ were examined using ELISA. All data are presented as the $M \pm S E M$ ( $n=3$ in each group). ${ }^{*} p<0.05$ vs. PBS group, ${ }^{*} p<0.01$ vs. PBS group, $\# p<0.05$ vs. H2O2 group, $\# \# p<0.01$ vs. $\mathrm{H} 2 \mathrm{O} 2$ group, \&p<0.05 vs. $\mathrm{H} 2 \mathrm{O} 2+$ si-NC group, \&\&p<0.01 vs. $\mathrm{H} 2 \mathrm{O} 2+$ si-NC group by ANOVA.
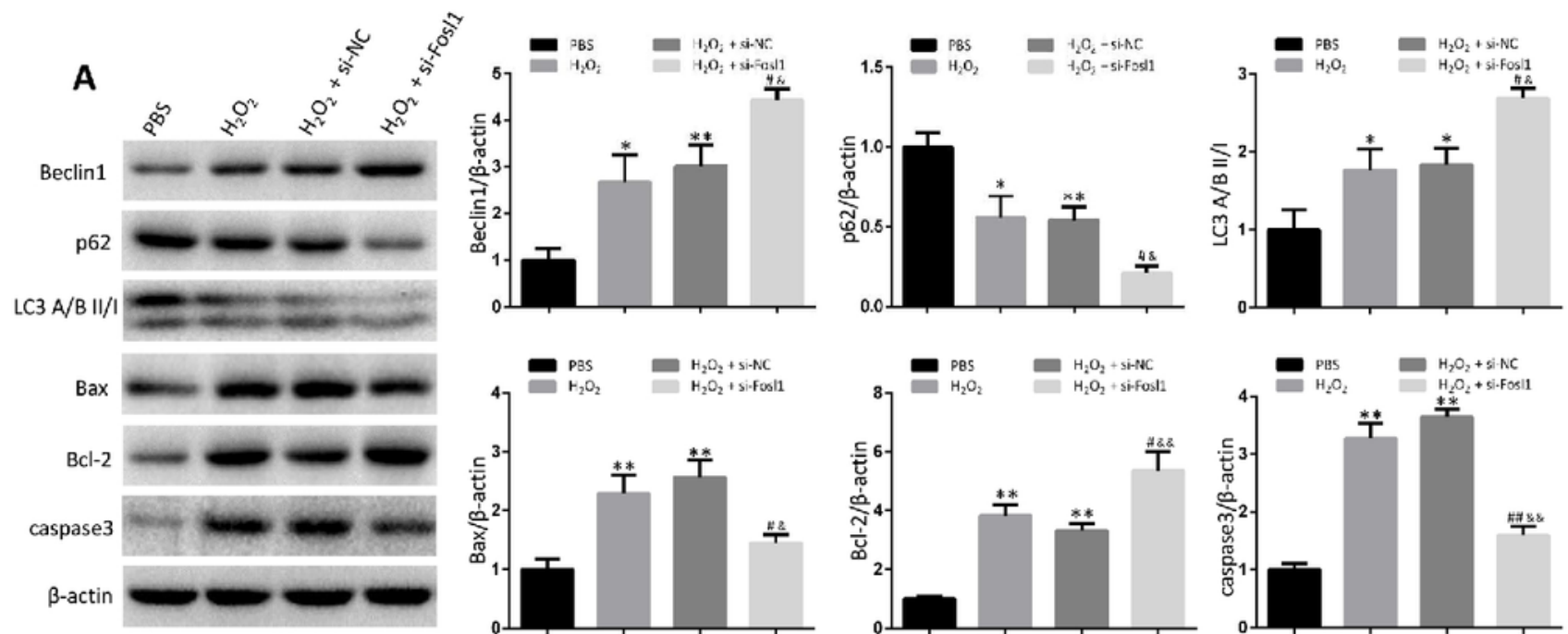

B
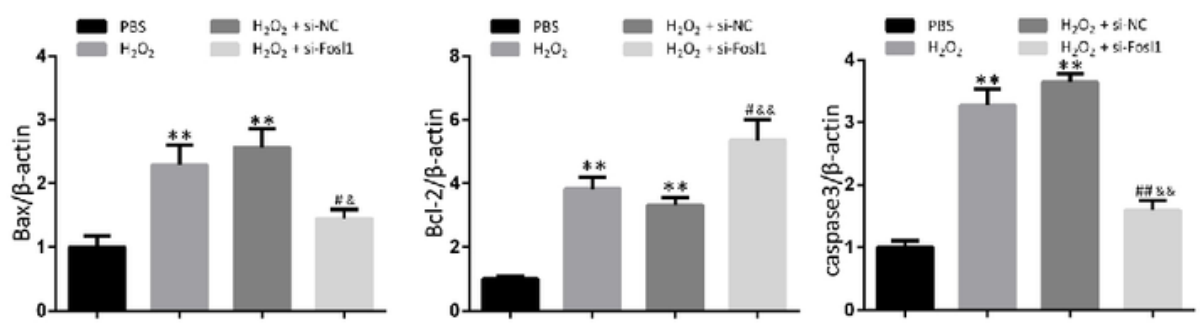

PBS
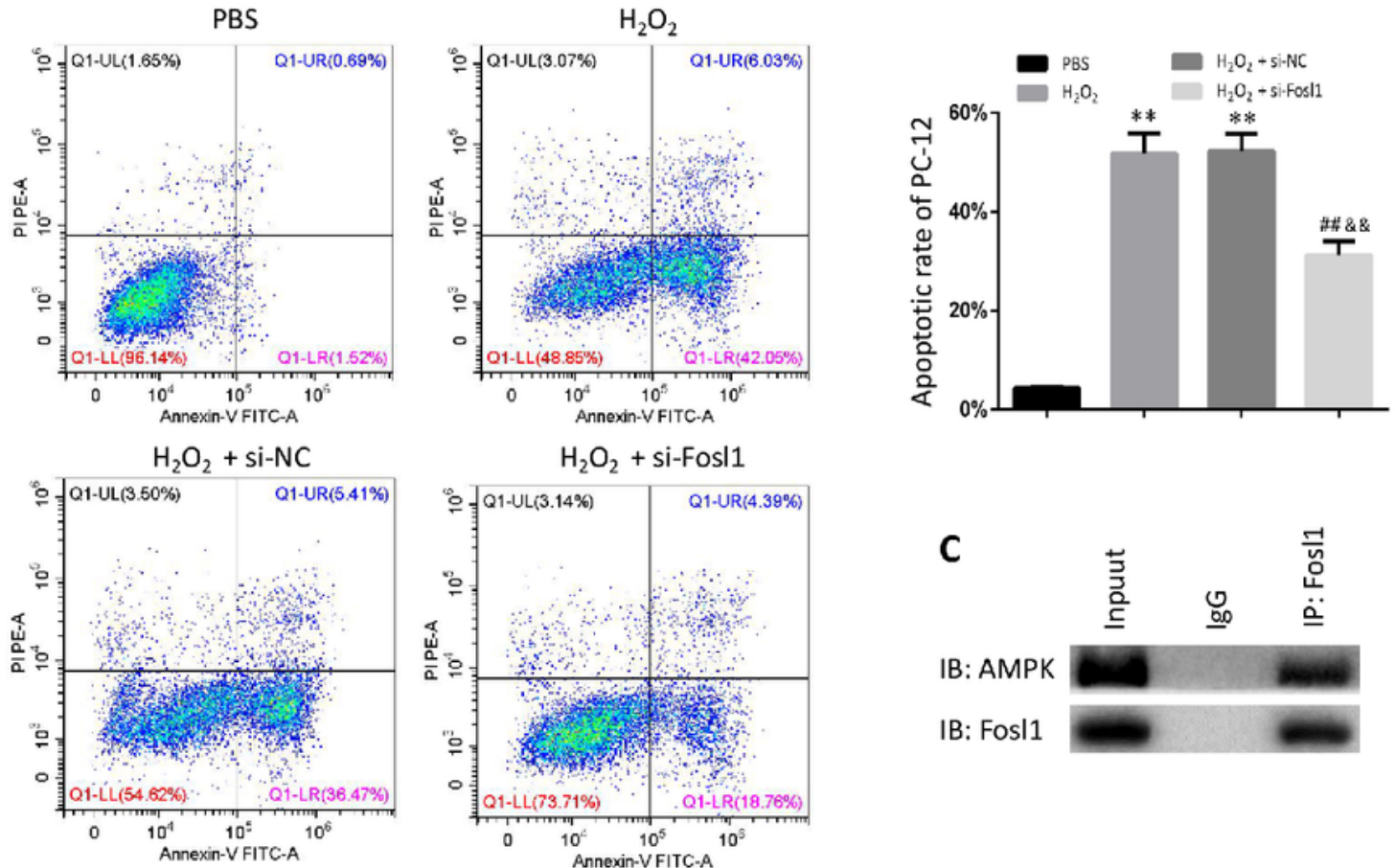

C

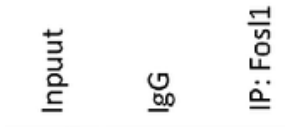

IB: AMPK

IB: Fosl1
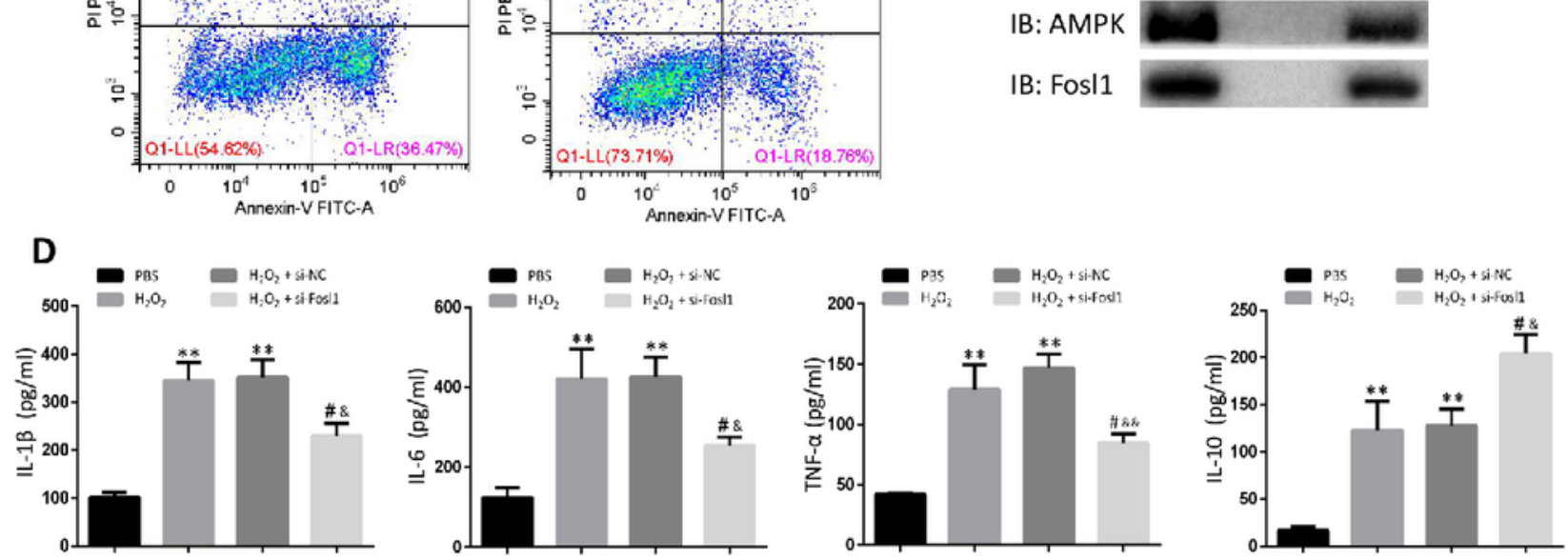

Figure 5 
si-Fosl1 enhanced the autophagic activity and decreased the apoptosis and inflammation. A: The autophagic marker proteins (Beclin1, p62, LC3) and the apoptotic proteins (Bax, Bcl-2, caspase3) were detected using western blotting. B: The apoptotic cell rate was monitored by flow cytometry. C: The COIP was used to examine the interaction of Fosl 1 and AMPK in injured PC-12 cells. D: The inflammatory proteins (IL-1 $\beta$, IL-6, TNF-a, IL-10) were examined using ELISA. All data are presented as the $\mathrm{M} \pm \mathrm{SEM}$ ( $n=3$ in each group). ${ }^{\star} p<0.05$ vs. PBS group, ${ }^{*} p<0.01$ vs. PBS group, $\# p<0.05$ vs. $\mathrm{H} 202$ group, $\# \# p<0.01$ vs. $\mathrm{H} 2 \mathrm{O} 2$ group, \&p<0.05 vs. $\mathrm{H} 2 \mathrm{O} 2+$ si-NC group, \&\&p<0.01 vs. $\mathrm{H} 2 \mathrm{O} 2+$ si-NC group by ANOVA.
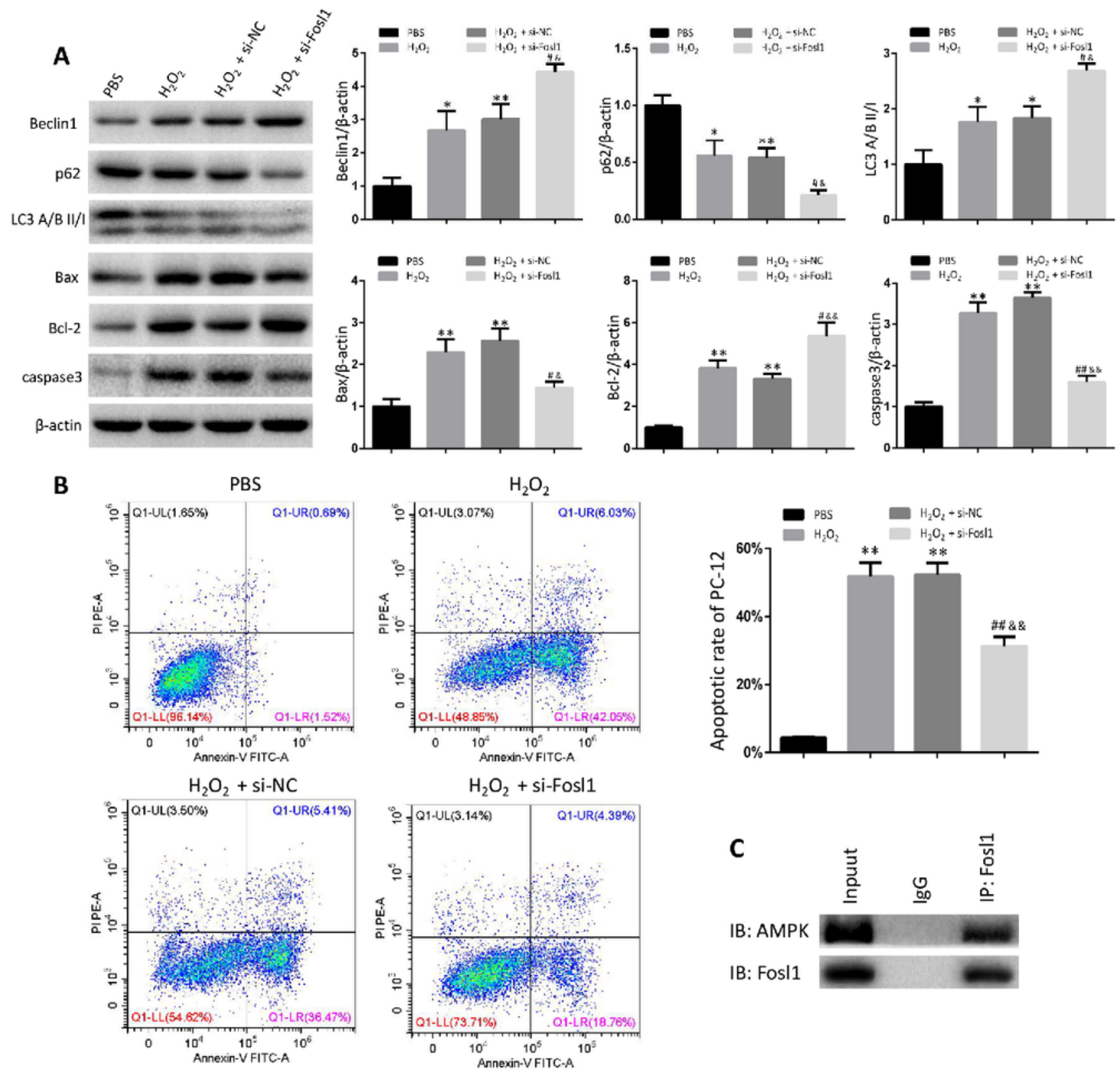

IB: AMPK

IB: Fosl1
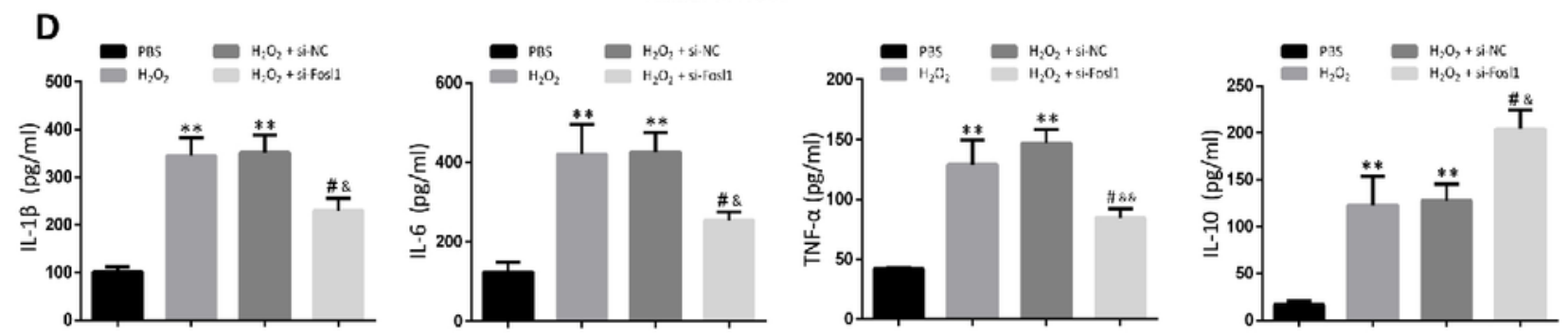


\section{Figure 5}

si-Fosl1 enhanced the autophagic activity and decreased the apoptosis and inflammation. A: The autophagic marker proteins (Beclin1, p62, LC3) and the apoptotic proteins (Bax, Bcl-2, caspase3) were detected using western blotting. B: The apoptotic cell rate was monitored by flow cytometry. C: The COIP was used to examine the interaction of Fosl 1 and AMPK in injured PC-12 cells. D: The inflammatory proteins (IL-1 $\beta$, IL-6, TNF-a, IL-10) were examined using ELISA. All data are presented as the $\mathrm{M} \pm \mathrm{SEM}$ ( $n=3$ in each group). ${ }^{*} p<0.05$ vs. PBS group, ${ }^{*} \mathrm{p}<00.01$ vs. PBS group, $\# p<0.05$ vs. H2O2 group, $\# \# p<0.01$ vs. $\mathrm{H} 2 \mathrm{O} 2$ group, \&p<0.05 vs. $\mathrm{H} 2 \mathrm{O} 2+$ si-NC group, \&\&p<0.01 vs. $\mathrm{H} 2 \mathrm{O} 2+$ si-NC group by ANOVA.

A

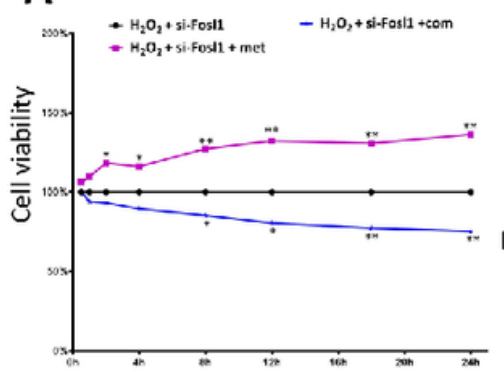

$\beta$-actin
B
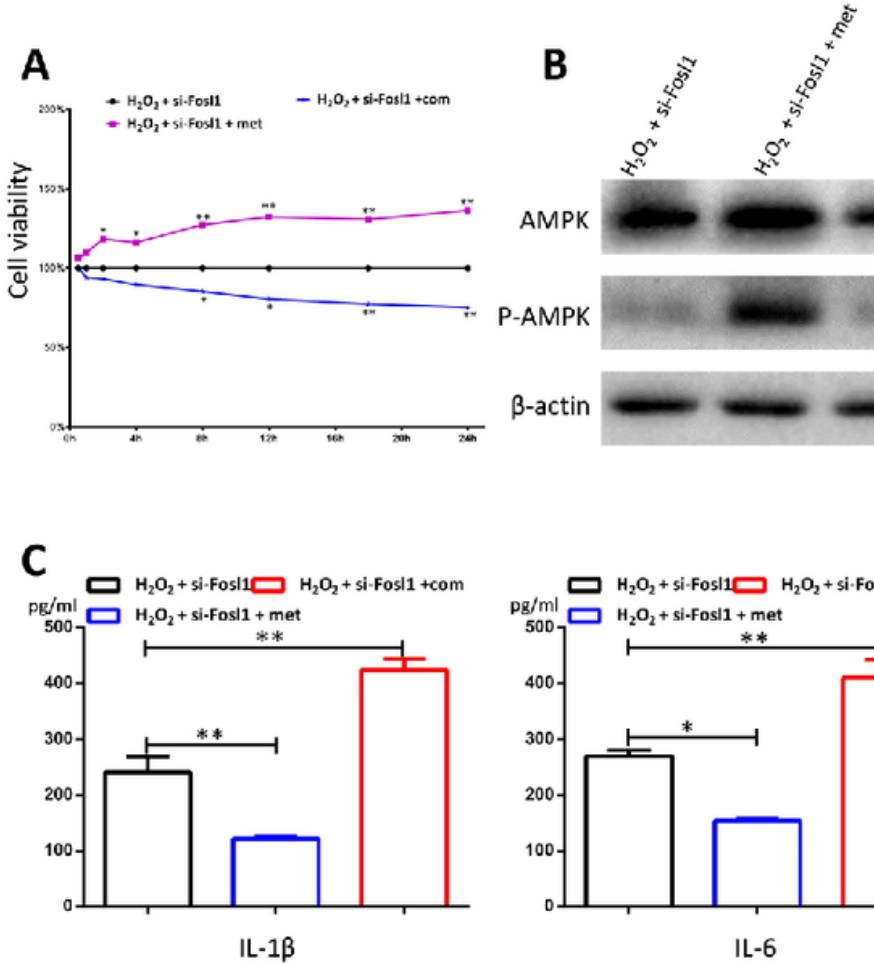
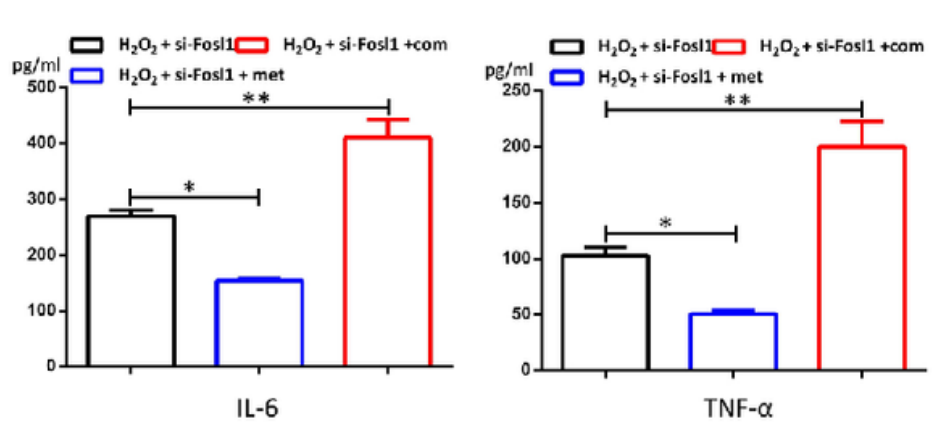

TNF- $\alpha$
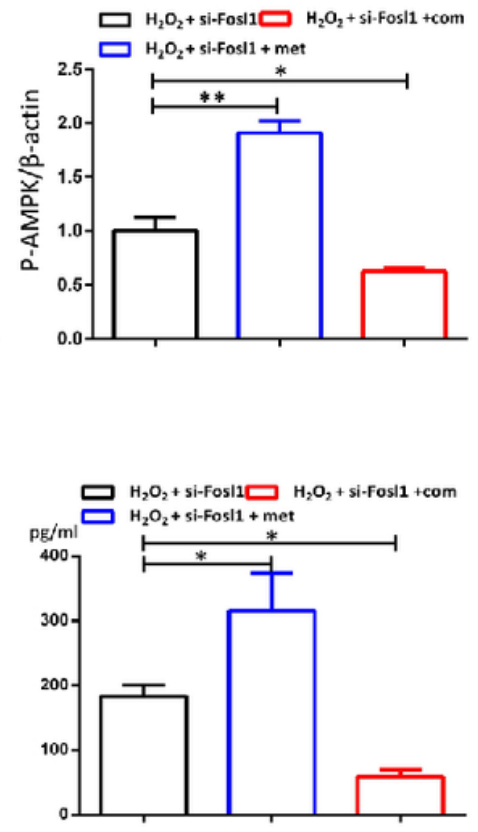

IL-10

\section{Figure 6}

An AMPK activator increased AMPK expression, promoted the phosphorylation of AMPK, improved the cells' viability and alleviated inflammation in the injured PC-12 cells with si-Fos 1 treatment. However, the AMPK inhibitor exhibited the opposite effects. A: Cell viability was checked by CCK-8 kits. B: AMPK and pAMPK were examined using western blotting. $C$ : The inflammatory marker proteins were checked using ELISA kits. All data are presented as the $\mathrm{M} \pm \mathrm{SEM}$ ( $\mathrm{n}=3$ in each group). ${ }^{\star} \mathrm{p}<0.05$ vs. $\mathrm{H} 2 \mathrm{O} 2+\mathrm{si-Fos} 11$ group, ${ }^{* *} \mathrm{p}<0.01$ vs. $\mathrm{H} 2 \mathrm{O} 2+$ si-Fosl1 group ( $\mathrm{n}=3$ ) by ANOVA. 
A
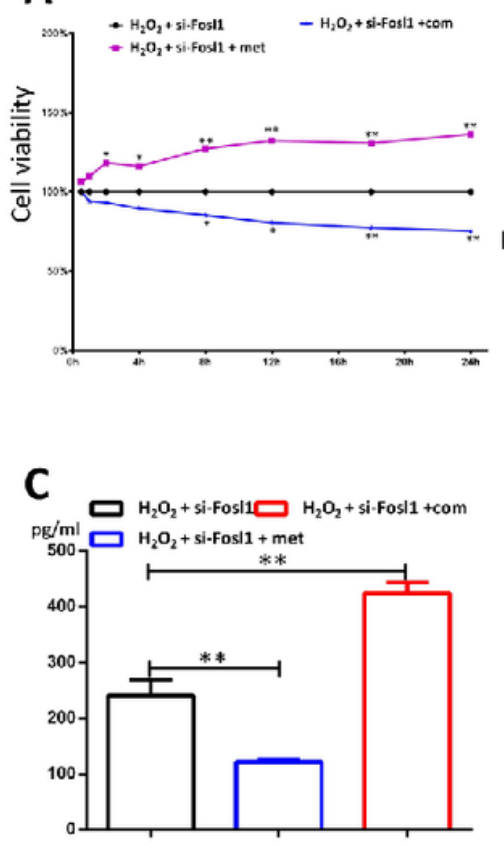

IL-1B
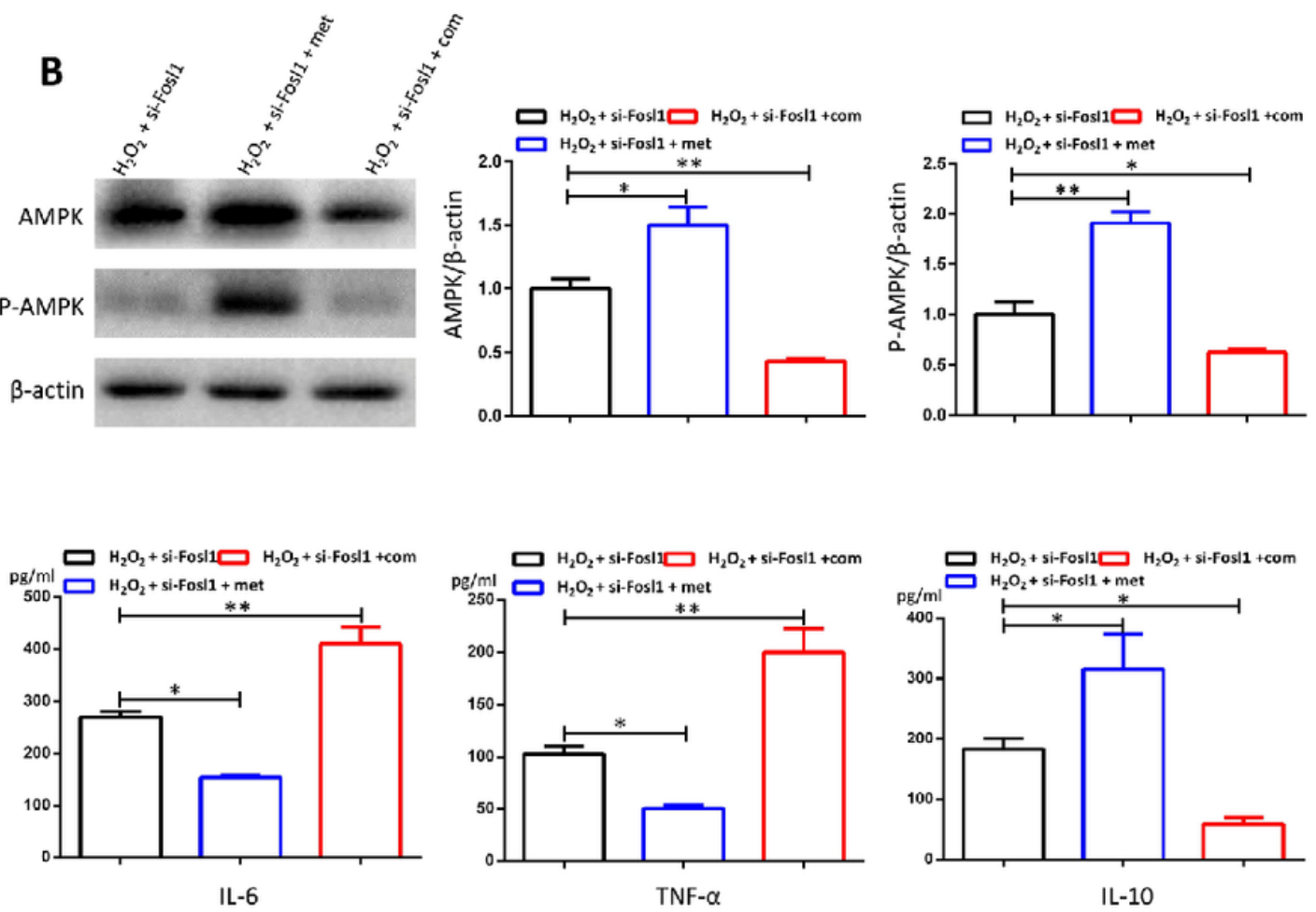

\section{Figure 6}

An AMPK activator increased AMPK expression, promoted the phosphorylation of AMPK, improved the cells' viability and alleviated inflammation in the injured PC-12 cells with si-Fos 1 treatment. However, the AMPK inhibitor exhibited the opposite effects. A: Cell viability was checked by CCK-8 kits. B: AMPK and pAMPK were examined using western blotting. C: The inflammatory marker proteins were checked using ELISA kits. All data are presented as the $\mathrm{M} \pm \mathrm{SEM}$ ( $\mathrm{n}=3$ in each group). ${ }^{\star} \mathrm{p}<0.05 \mathrm{vs}$. $\mathrm{H} 2 \mathrm{O} 2+$ si-Fosl 1 group, $\star * \mathrm{p}<0.01$ vs. $\mathrm{H} 2 \mathrm{O} 2+$ si-Fosl1 group $(\mathrm{n}=3)$ by ANOVA. 
A
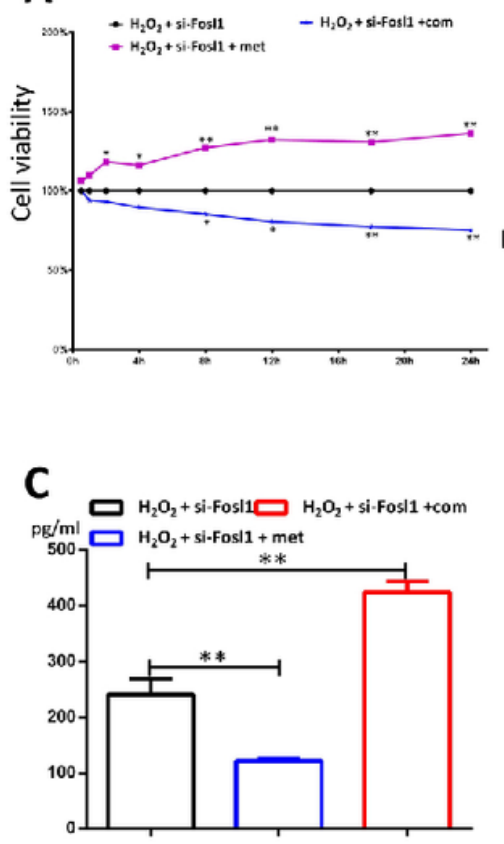

IL-1B
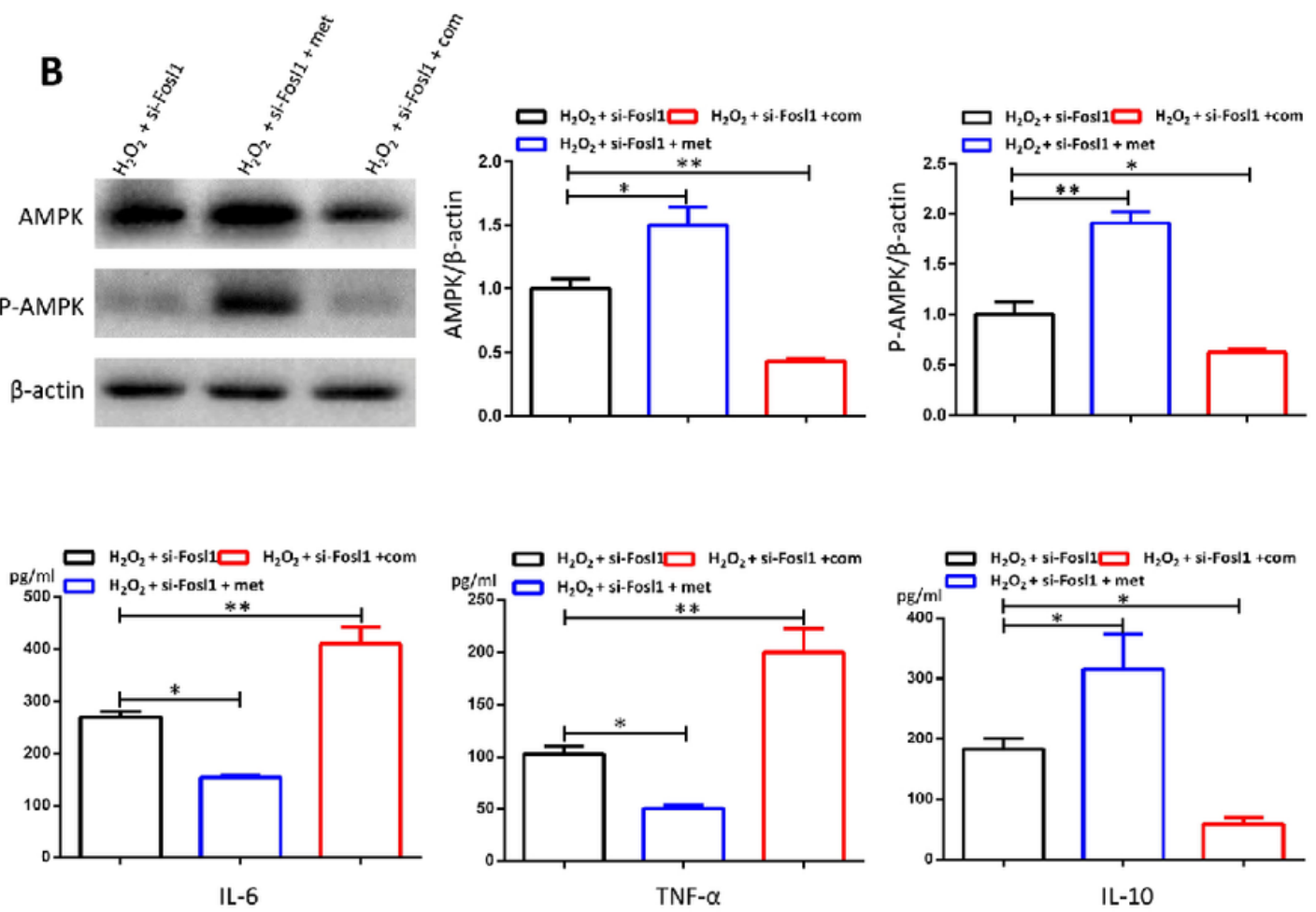

\section{Figure 6}

An AMPK activator increased AMPK expression, promoted the phosphorylation of AMPK, improved the cells' viability and alleviated inflammation in the injured PC-12 cells with si-Fos 1 treatment. However, the AMPK inhibitor exhibited the opposite effects. A: Cell viability was checked by CCK-8 kits. B: AMPK and pAMPK were examined using western blotting. C: The inflammatory marker proteins were checked using ELISA kits. All data are presented as the $\mathrm{M} \pm \mathrm{SEM}$ ( $\mathrm{n}=3$ in each group). ${ }^{\star} \mathrm{p}<0.05 \mathrm{vs}$. $\mathrm{H} 2 \mathrm{O} 2+$ si-Fosl 1 group, $\star * p<0.01$ vs. $\mathrm{H} 2 \mathrm{O} 2+$ si-Fosl1 group $(\mathrm{n}=3)$ by ANOVA. 
A
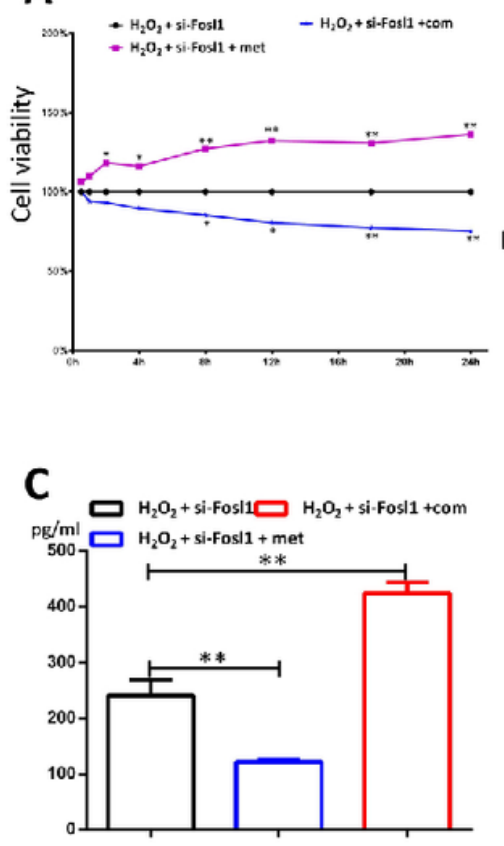

IL-1B
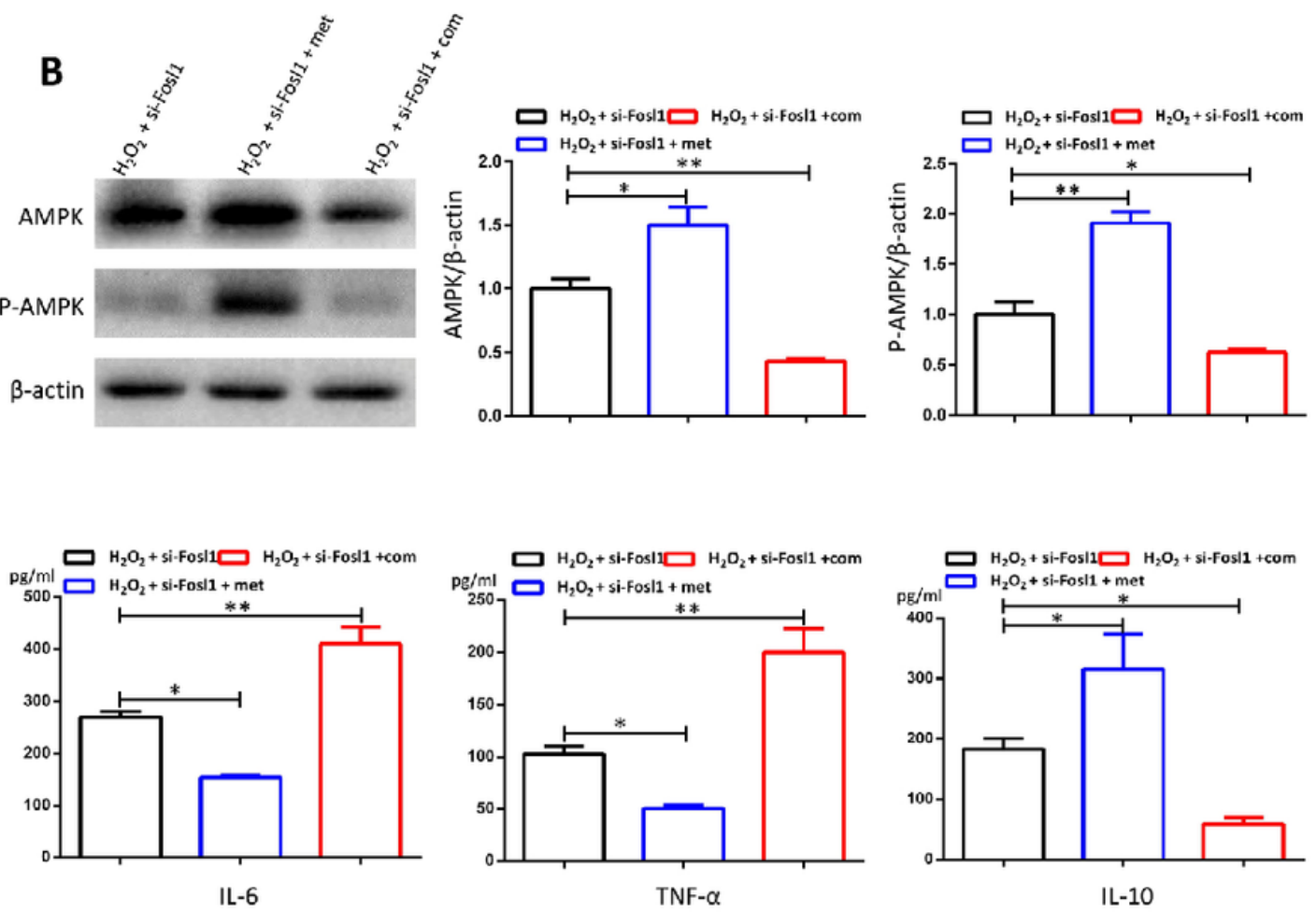

\section{Figure 6}

An AMPK activator increased AMPK expression, promoted the phosphorylation of AMPK, improved the cells' viability and alleviated inflammation in the injured PC-12 cells with si-Fos 1 treatment. However, the AMPK inhibitor exhibited the opposite effects. A: Cell viability was checked by CCK-8 kits. B: AMPK and pAMPK were examined using western blotting. C: The inflammatory marker proteins were checked using ELISA kits. All data are presented as the $\mathrm{M} \pm \mathrm{SEM}$ ( $\mathrm{n}=3$ in each group). ${ }^{\star} \mathrm{p}<0.05 \mathrm{vs}$. $\mathrm{H} 2 \mathrm{O} 2+$ si-Fosl 1 group, $\star * p<0.01$ vs. $\mathrm{H} 2 \mathrm{O} 2+$ si-Fosl1 group $(\mathrm{n}=3)$ by ANOVA. 


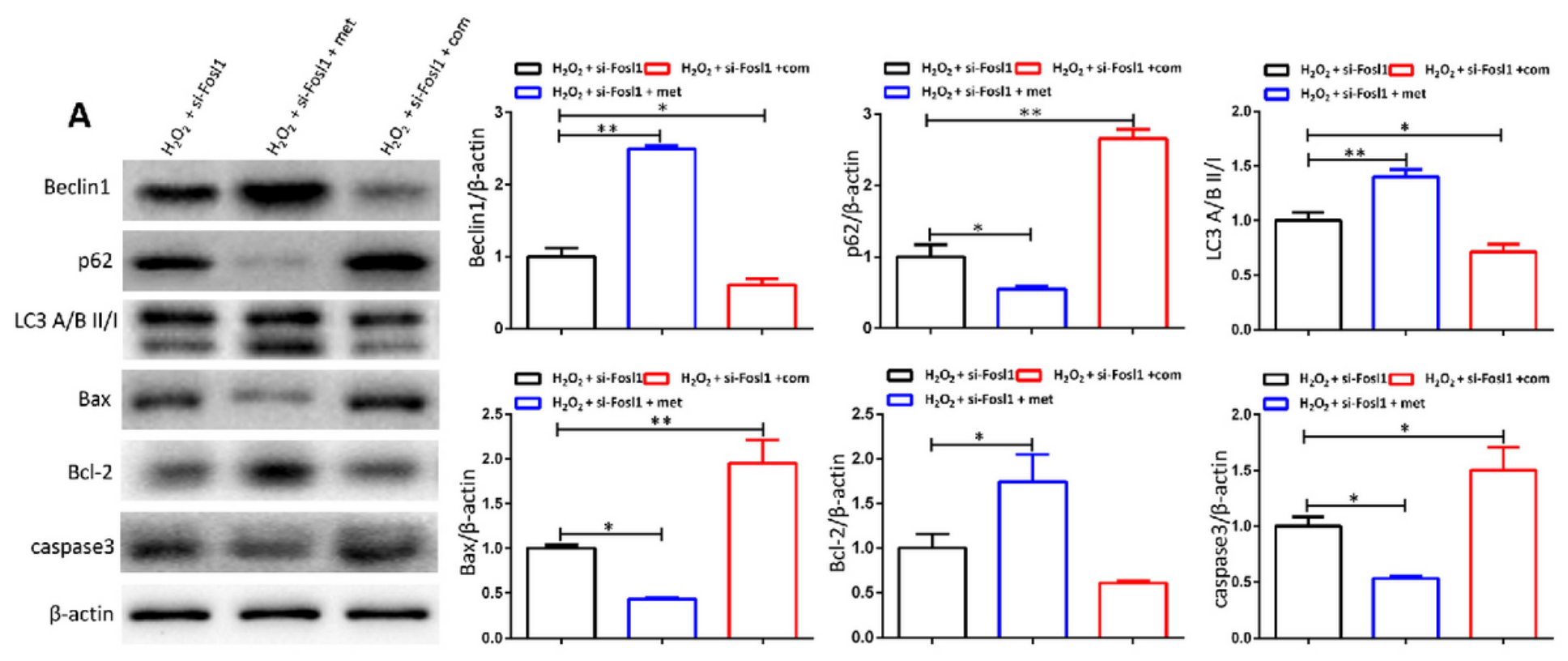

B $\quad \mathrm{H}_{2} \mathrm{O}_{2}+\mathrm{si}$-Fosl1

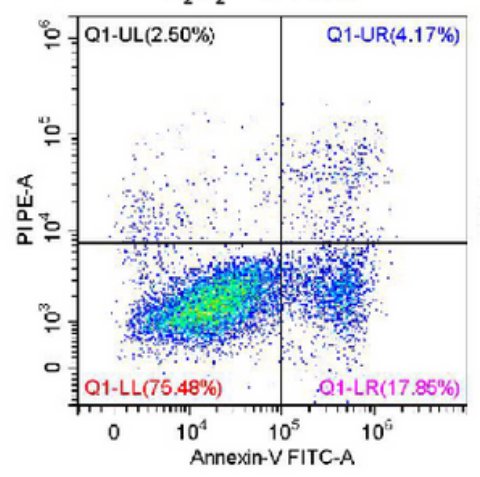

$\mathrm{H}_{2} \mathrm{O}_{2}+$ si-Fosl1 + met

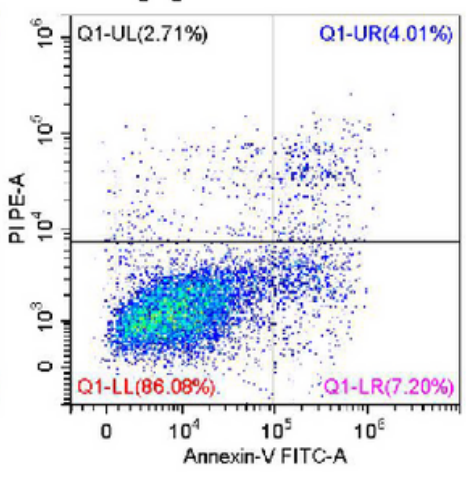

$\mathrm{H}_{2} \mathrm{O}_{2}+$ si-Fosl1 + com

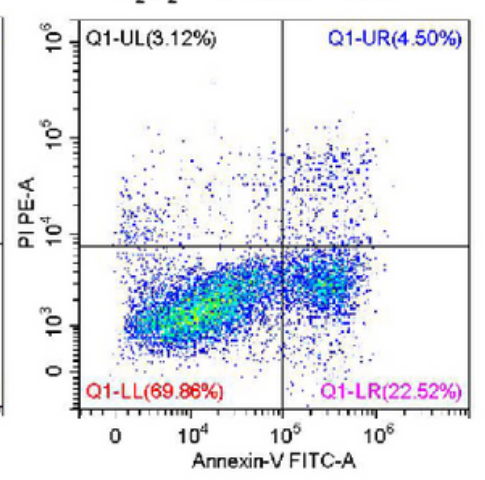

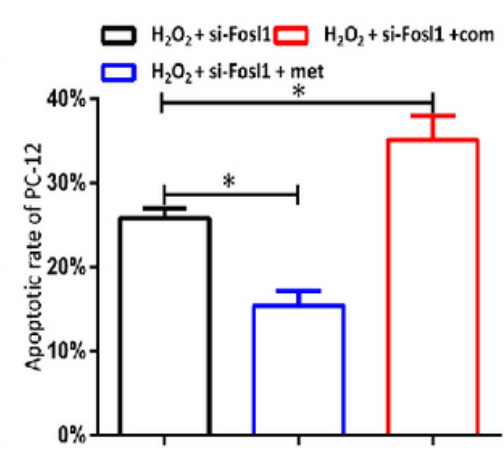

Figure 7

The AMPK activator increased the autophagic activity and decreased the apoptotic proteins and apoptotic rate in the injured PC-12 cells with si-Fosl1 treatment. An AMPK inhibitor suppressed autophagy and elevated apoptotic protein expression and the apoptotic rate. A: The autophagic marker proteins (Beclin1, p62, LC3) and the apoptotic proteins (Bax, Bcl-2, caspase3) were measured using western blotting. B: The apoptotic cell rate was examined by flow cytometry. All data are presented as the $\mathrm{M} \pm$ SEM ( $n=3$ in each group). ${ }^{*} p<0.05$ vs. $\mathrm{H} 2 \mathrm{O} 2+$ si-Fosl1 group, ${ }^{\star *} \mathrm{p}<0.01$ vs. $\mathrm{H} 2 \mathrm{O} 2+$ si-Fosl1 group $(n=3)$ by ANOVA. 


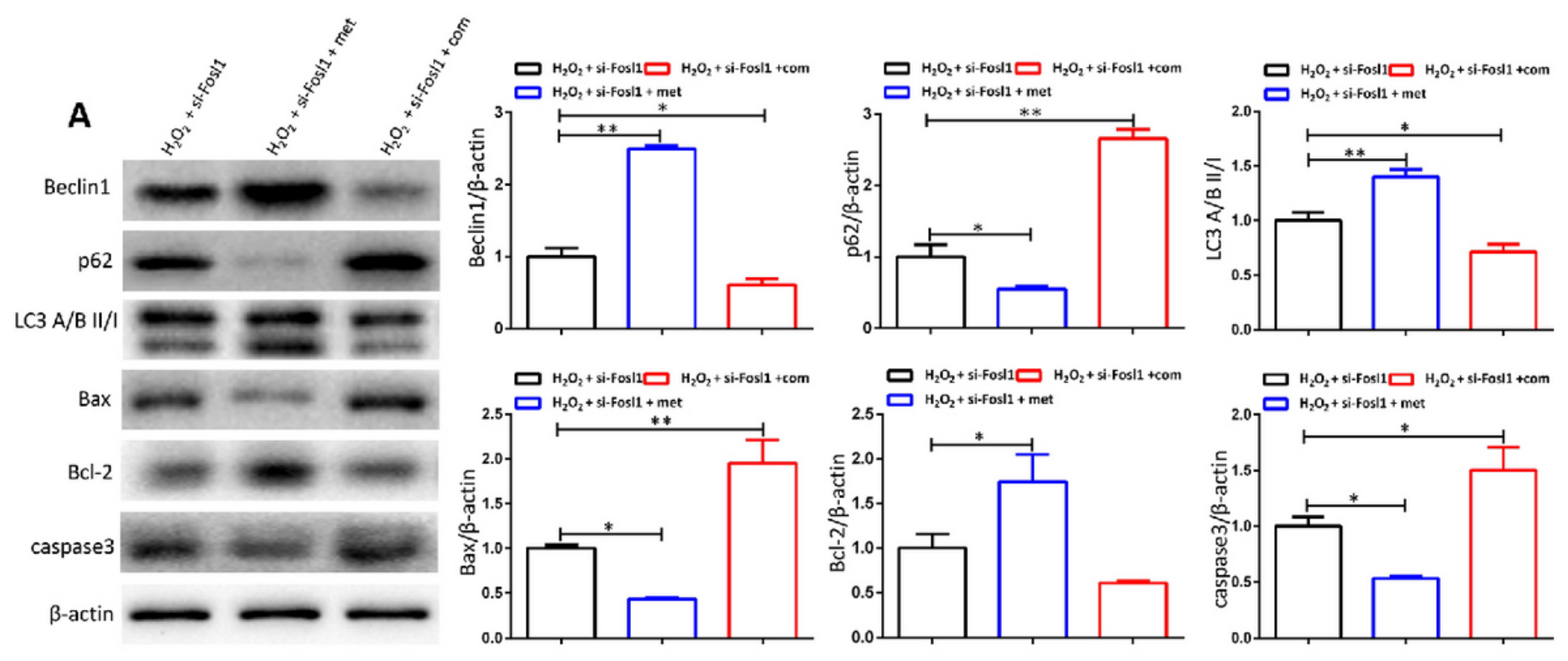

B $\quad \mathrm{H}_{2} \mathrm{O}_{2}+\mathrm{si}$-Fosl1

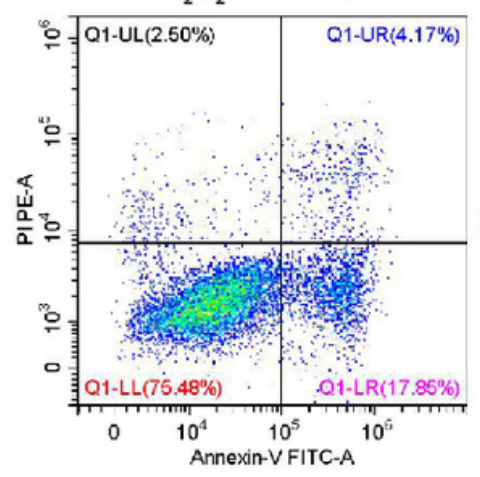

$\mathrm{H}_{2} \mathrm{O}_{2}+$ si-Fosl1 + met

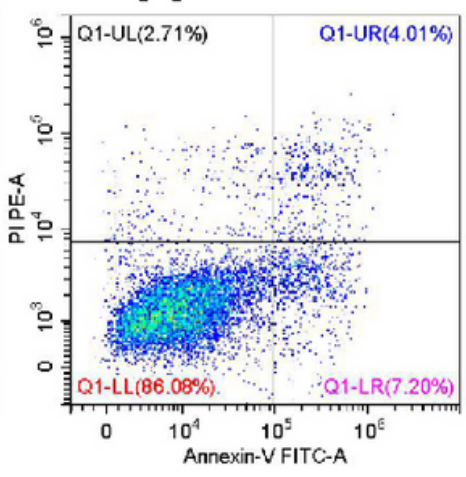

$\mathrm{H}_{2} \mathrm{O}_{2}+$ si-Fosl1 + com

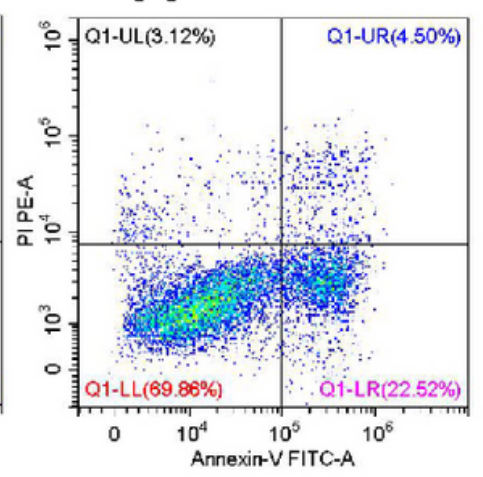

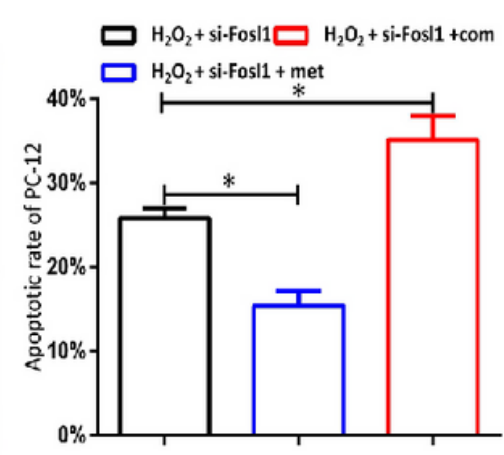

Figure 7

The AMPK activator increased the autophagic activity and decreased the apoptotic proteins and apoptotic rate in the injured PC-12 cells with si-Fosl1 treatment. An AMPK inhibitor suppressed autophagy and elevated apoptotic protein expression and the apoptotic rate. A: The autophagic marker proteins (Beclin1, p62, LC3) and the apoptotic proteins (Bax, Bcl-2, caspase3) were measured using western blotting. B: The apoptotic cell rate was examined by flow cytometry. All data are presented as the $\mathrm{M} \pm$ SEM ( $n=3$ in each group). ${ }^{*} p<0.05$ vs. $\mathrm{H} 2 \mathrm{O} 2+$ si-Fosl1 group, ${ }^{\star *} \mathrm{p}<0.01$ vs. $\mathrm{H} 2 \mathrm{O} 2+$ si-Fosl1 group $(n=3)$ by ANOVA. 


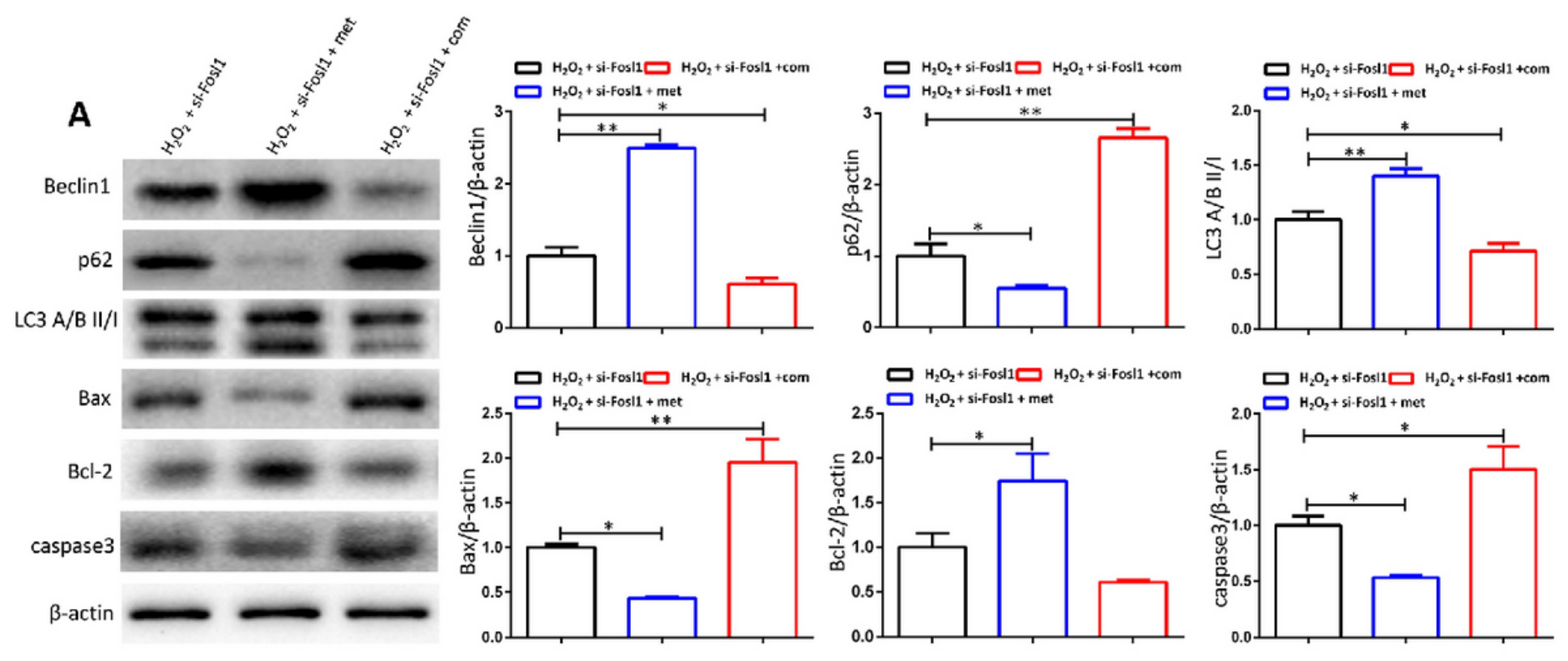

B $\quad \mathrm{H}_{2} \mathrm{O}_{2}+\mathrm{si}$-Fosl1
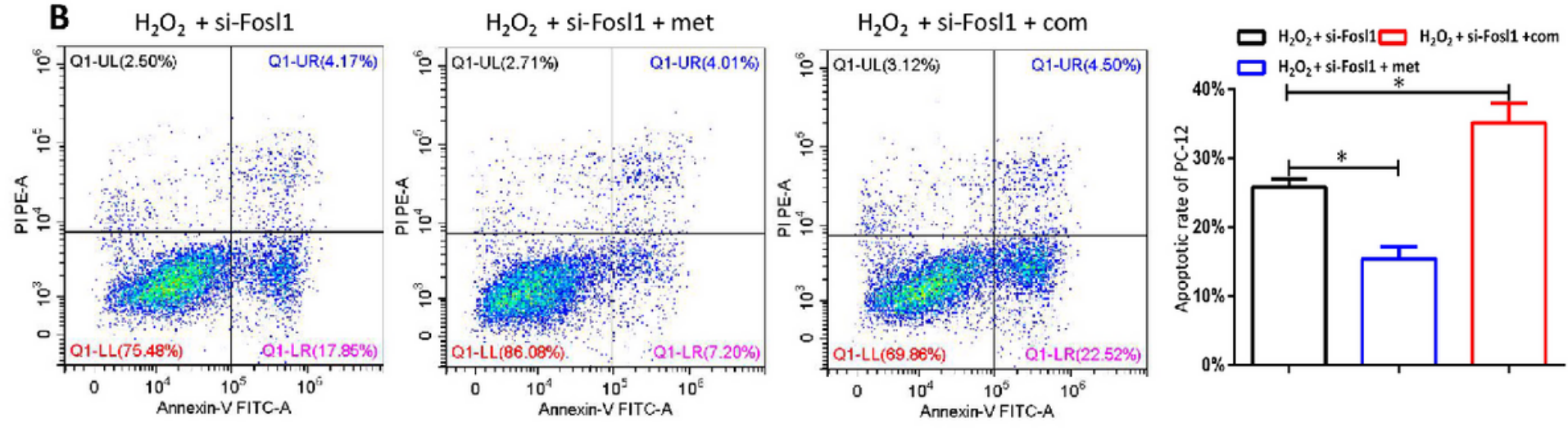

Figure 7

The AMPK activator increased the autophagic activity and decreased the apoptotic proteins and apoptotic rate in the injured PC-12 cells with si-Fosl1 treatment. An AMPK inhibitor suppressed autophagy and elevated apoptotic protein expression and the apoptotic rate. A: The autophagic marker proteins (Beclin1, p62, LC3) and the apoptotic proteins (Bax, Bcl-2, caspase3) were measured using western blotting. B: The apoptotic cell rate was examined by flow cytometry. All data are presented as the $\mathrm{M} \pm$ SEM ( $n=3$ in each group). ${ }^{\star} p<0.05$ vs. $\mathrm{H} 2 \mathrm{O} 2+$ si-Fosl1 group, ${ }^{\star *} \mathrm{p}<0.01$ vs. $\mathrm{H} 2 \mathrm{O} 2+$ si-Fosl1 group $(n=3)$ by ANOVA. 


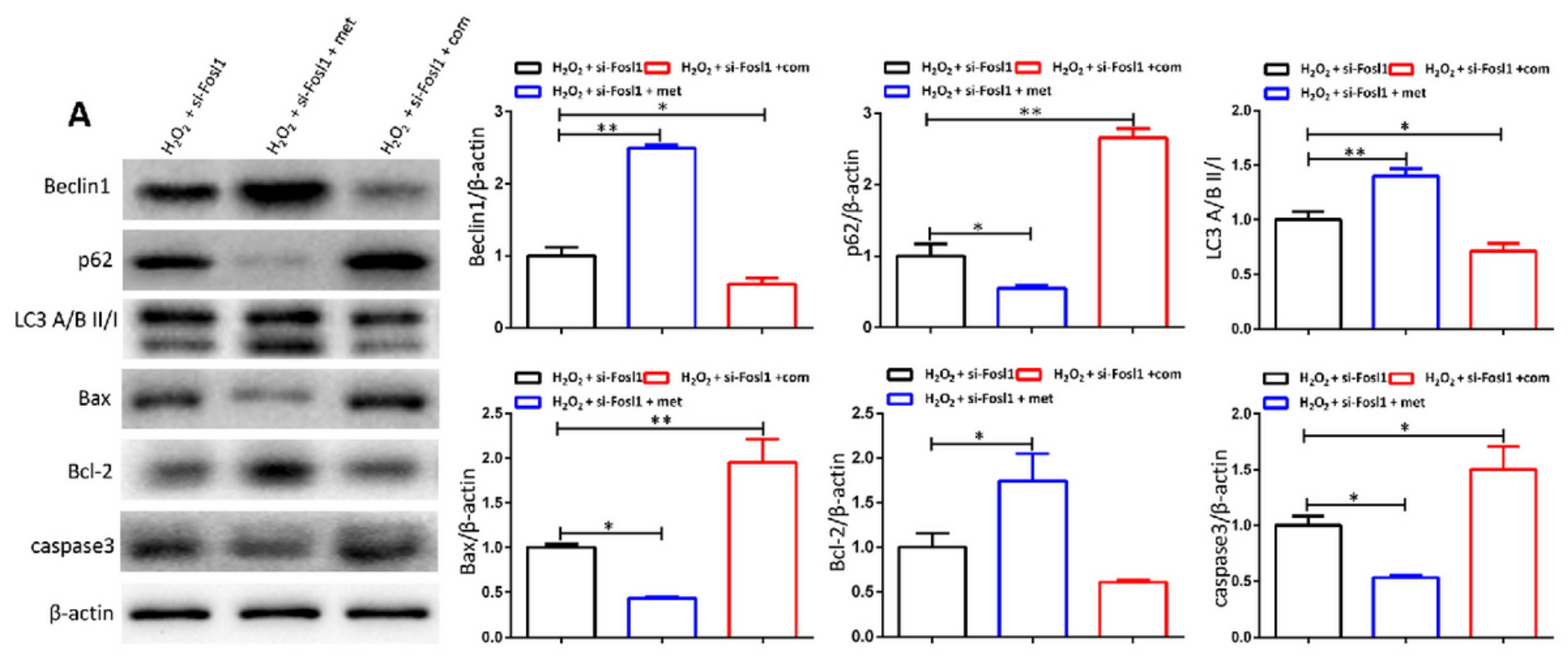

B $\quad \mathrm{H}_{2} \mathrm{O}_{2}+\mathrm{si}$-Fosl1
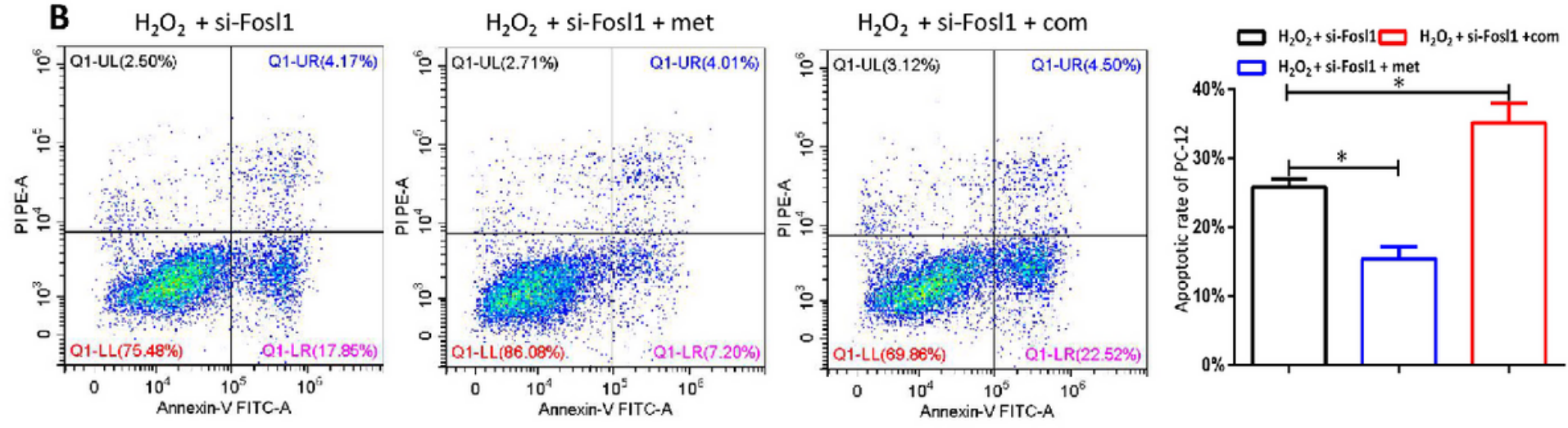

Figure 7

The AMPK activator increased the autophagic activity and decreased the apoptotic proteins and apoptotic rate in the injured PC-12 cells with si-Fosl1 treatment. An AMPK inhibitor suppressed autophagy and elevated apoptotic protein expression and the apoptotic rate. A: The autophagic marker proteins (Beclin1, p62, LC3) and the apoptotic proteins (Bax, Bcl-2, caspase3) were measured using western blotting. B: The apoptotic cell rate was examined by flow cytometry. All data are presented as the $\mathrm{M} \pm$ SEM ( $n=3$ in each group). ${ }^{\star} p<0.05$ vs. $\mathrm{H} 2 \mathrm{O} 2+$ si-Fosl1 group, ${ }^{\star *} \mathrm{p}<0.01$ vs. $\mathrm{H} 2 \mathrm{O} 2+$ si-Fosl1 group $(n=3)$ by ANOVA. 

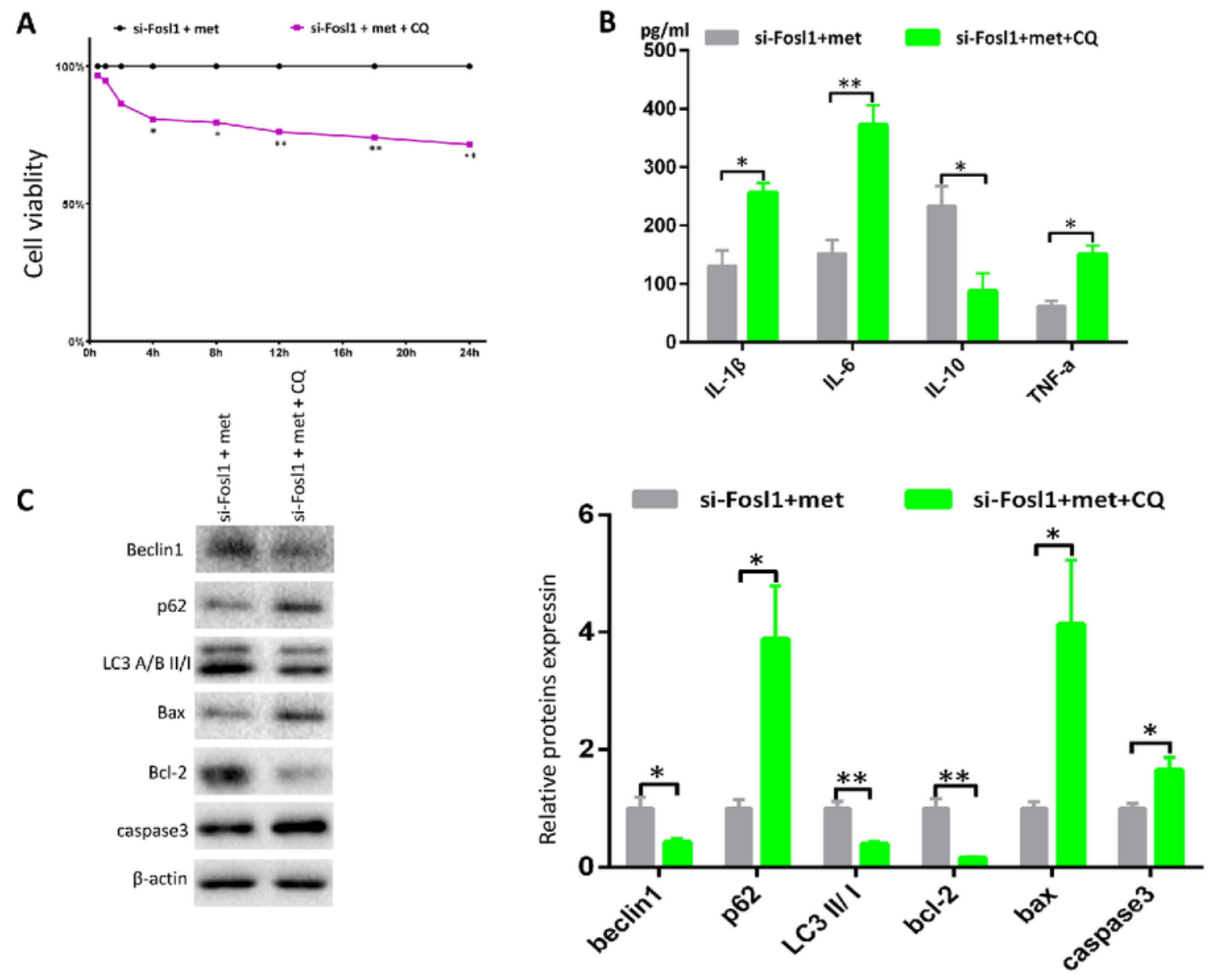

D
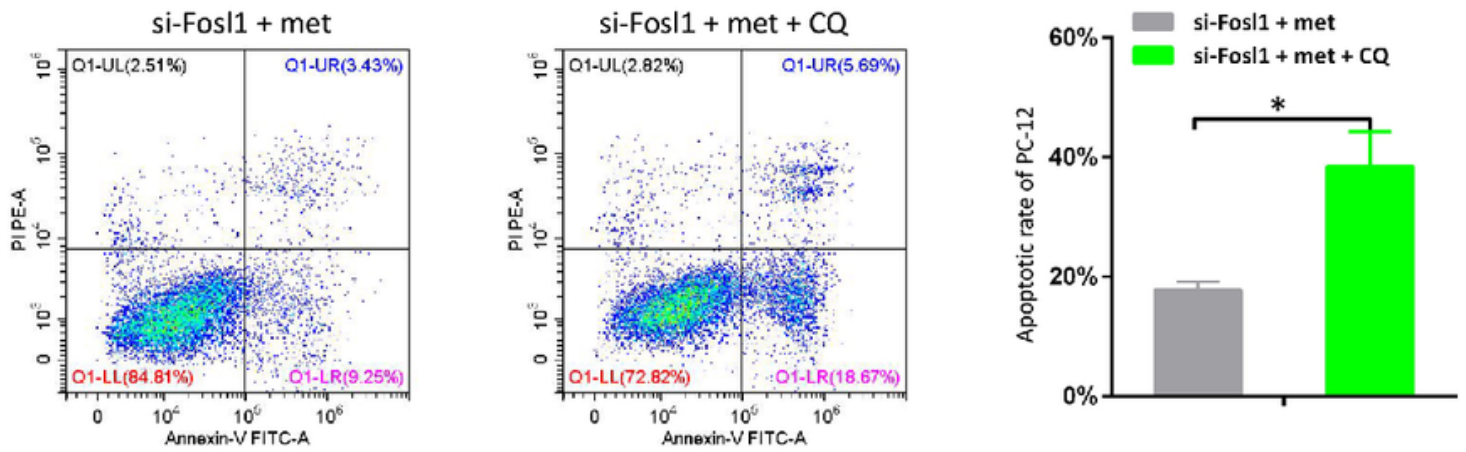

\section{Figure 8}

Inhibition of autophagy neutralized the benefits on the autophagic activity and cell viability after AMPK activation in the injured PC-12 cells with si-Fosl1 treatment but promoted inflammation and cell apoptosis. A: Cell viability was detected using CCK-8 kits in the control group and the CQ group. B: The inflammatory level was checked by ELISA kits. C: The autophagic and apoptotic proteins were visualized by western blotting. D: The cell apoptotic rate was inspected using flow cytometry. All data are presented 
as the $M \pm S E M\left(n=3\right.$ in each group). ${ }^{\star} p<0.05$ vs. si-Fosl $1+$ met group, ${ }^{\star \star} p<0.01$ si-Fosl $1+$ met group $(n=3)$ by $T$ test.
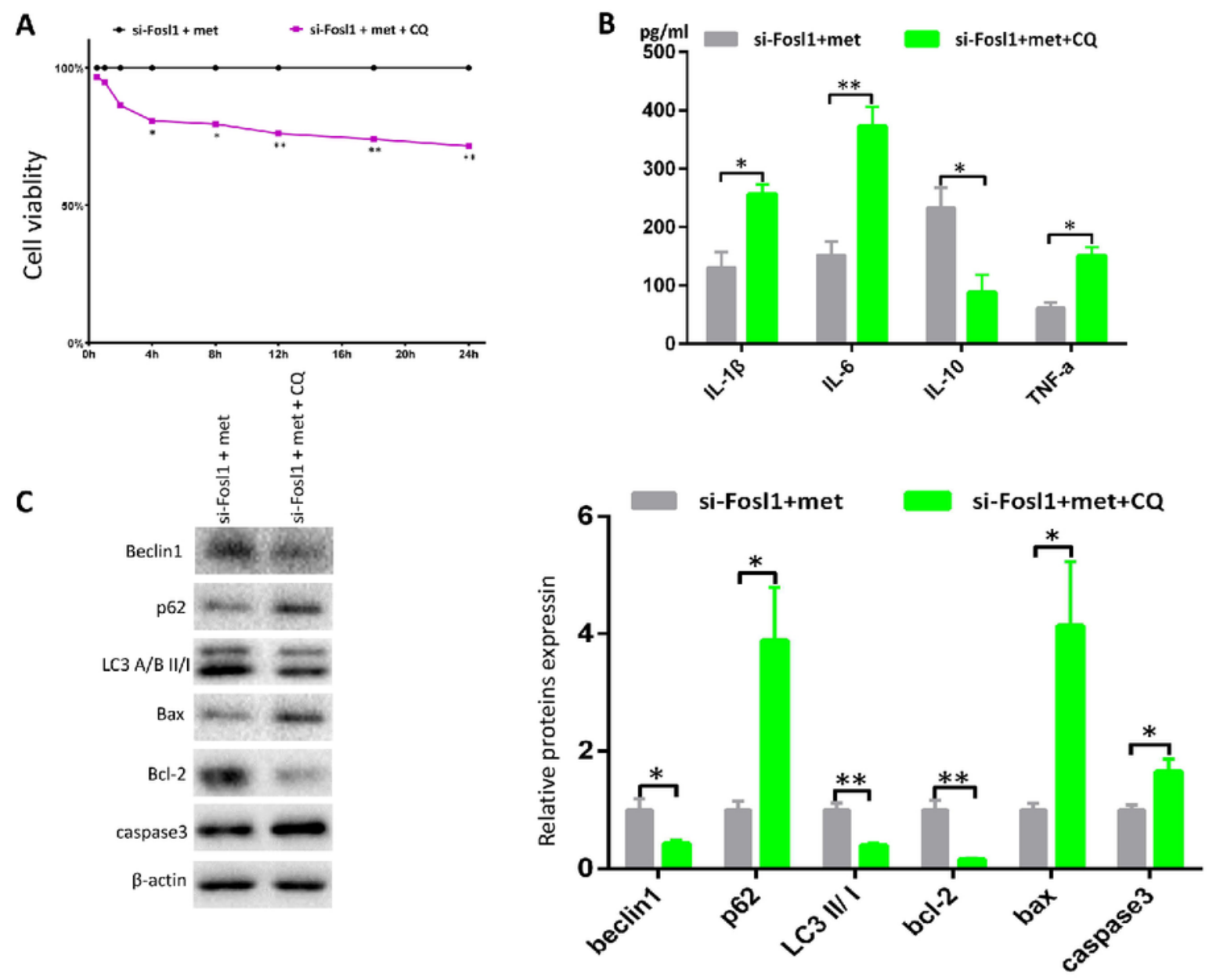

D
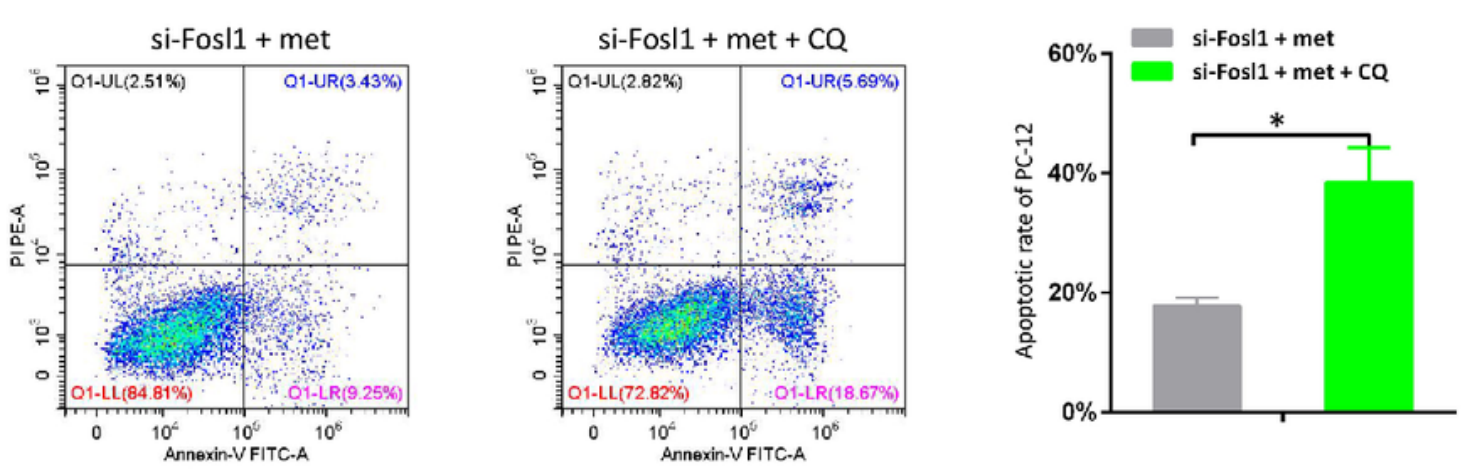

Figure 8

Inhibition of autophagy neutralized the benefits on the autophagic activity and cell viability after AMPK activation in the injured PC-12 cells with si-Fosl1 treatment but promoted inflammation and cell apoptosis. A: Cell viability was detected using CCK-8 kits in the control group and the CQ group. B: The 
inflammatory level was checked by ELISA kits. C: The autophagic and apoptotic proteins were visualized by western blotting. D: The cell apoptotic rate was inspected using flow cytometry. All data are presented as the $M \pm S E M\left(n=3\right.$ in each group). ${ }^{*} p<0.05$ vs. si-Fosl $1+$ met group, ${ }^{\star \star} p<0.01$ si-Fosl $1+$ met group $(n=3)$ by $T$ test.
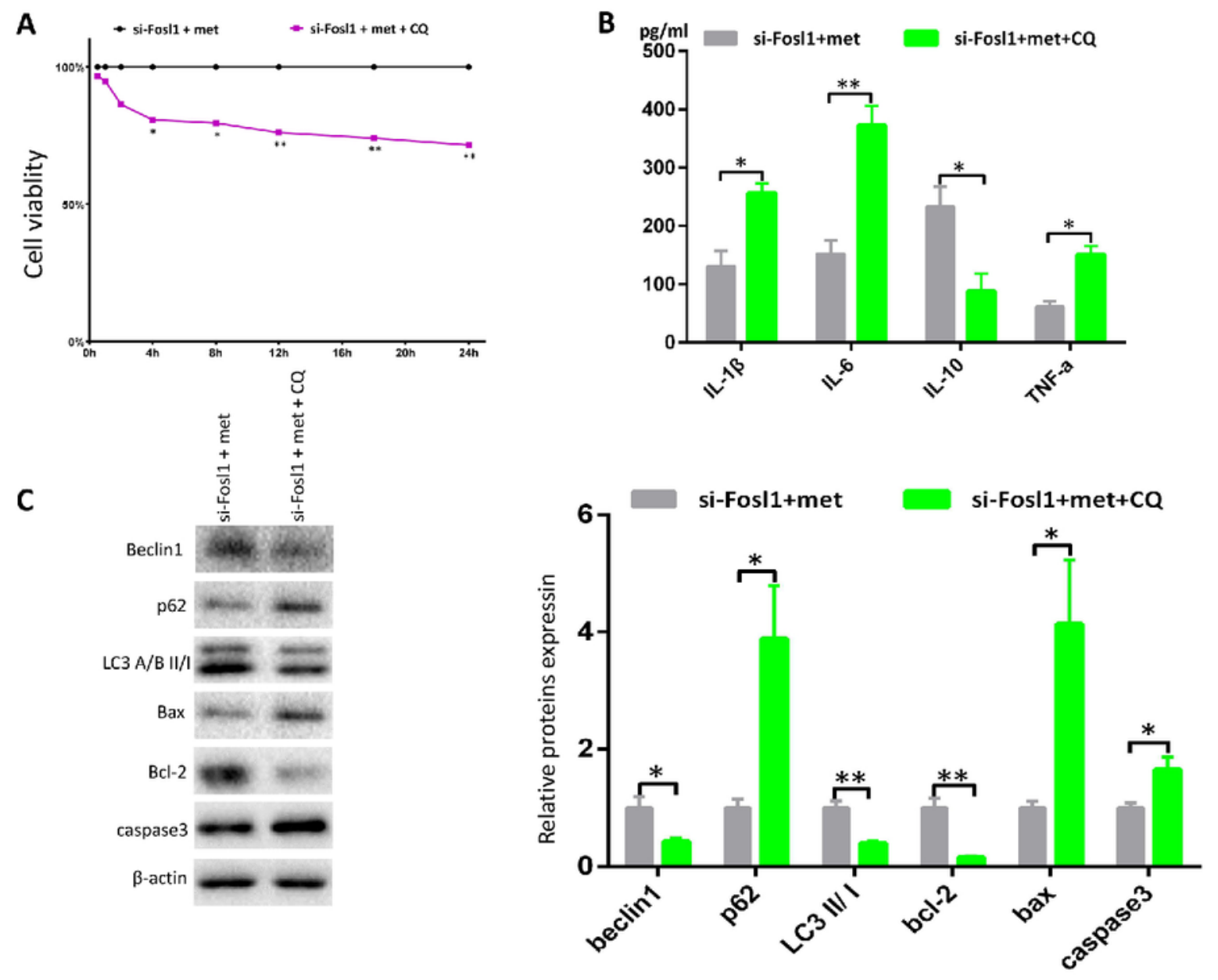

D
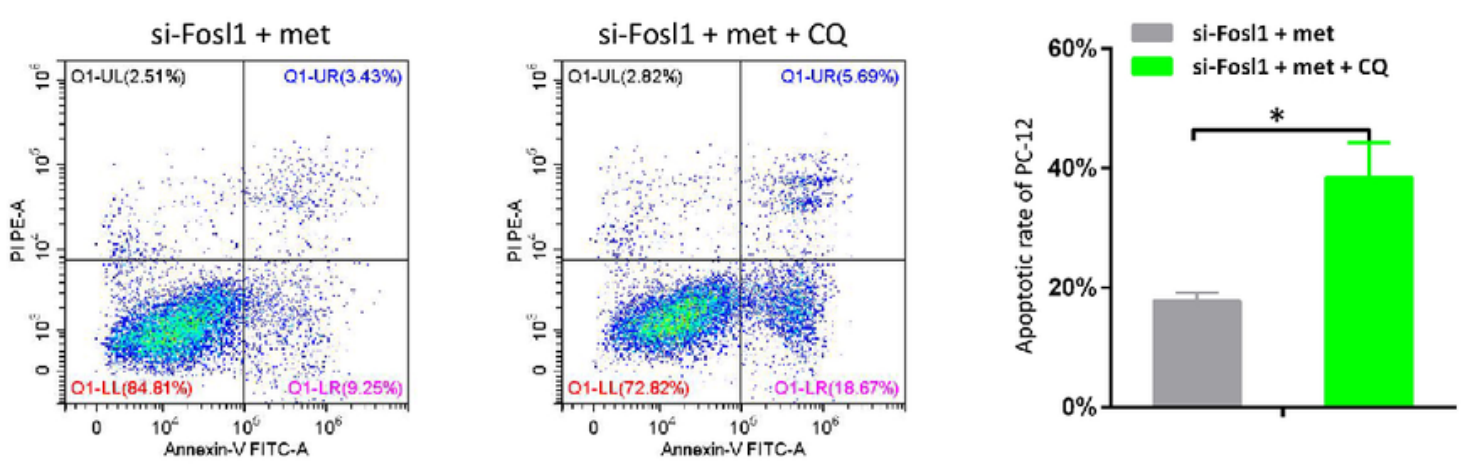

Figure 8 
Inhibition of autophagy neutralized the benefits on the autophagic activity and cell viability after AMPK activation in the injured PC-12 cells with si-Fosl1 treatment but promoted inflammation and cell apoptosis. A: Cell viability was detected using CCK-8 kits in the control group and the CQ group. B: The inflammatory level was checked by ELISA kits. C: The autophagic and apoptotic proteins were visualized by western blotting. D: The cell apoptotic rate was inspected using flow cytometry. All data are presented as the $M \pm S E M\left(n=3\right.$ in each group). ${ }^{*} p<0.05$ vs. si-Fosl $1+$ met group, ${ }^{\star *} p<0.01$ si-Fosl $1+$ met group $(n=3)$ by $T$ test.
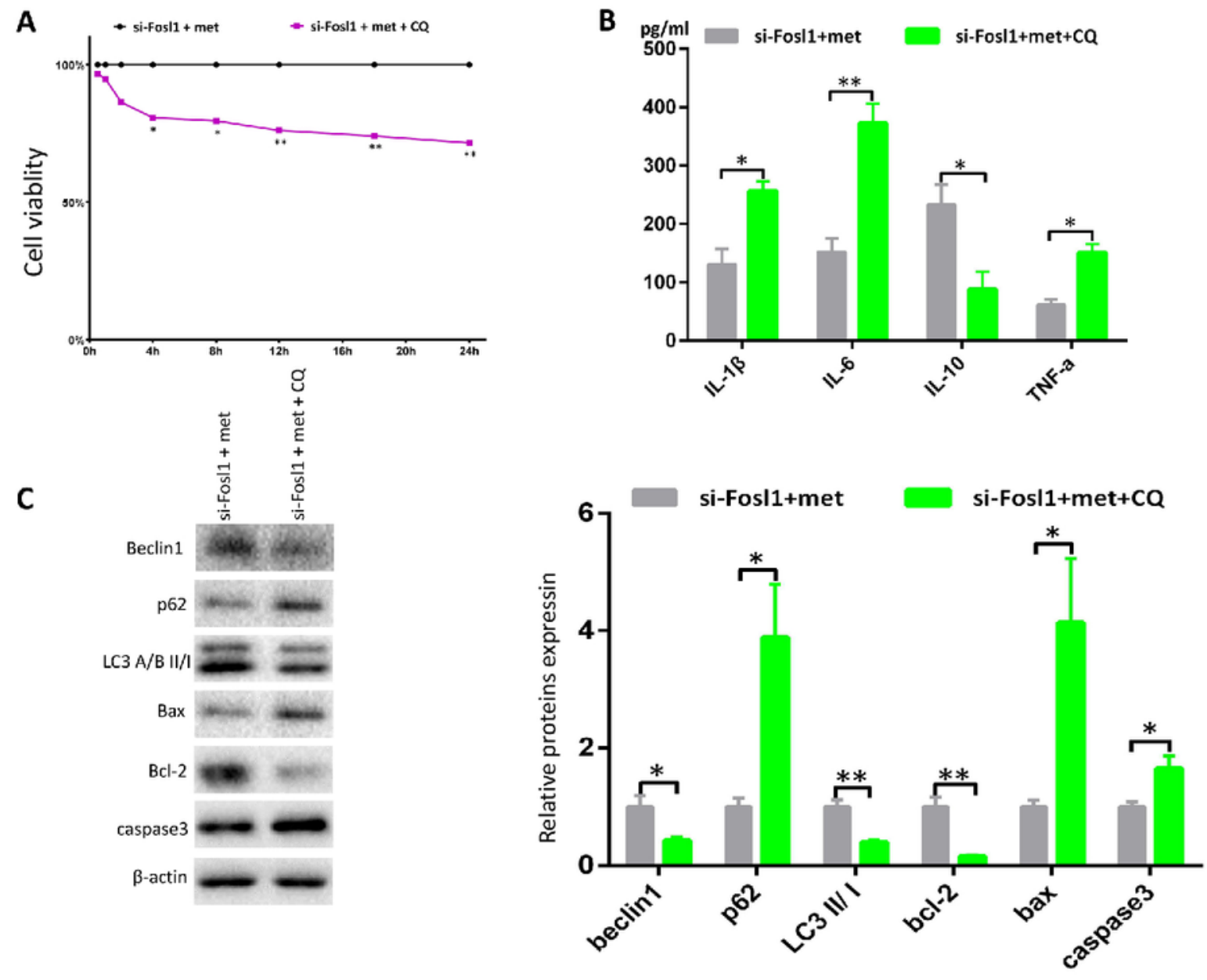

D
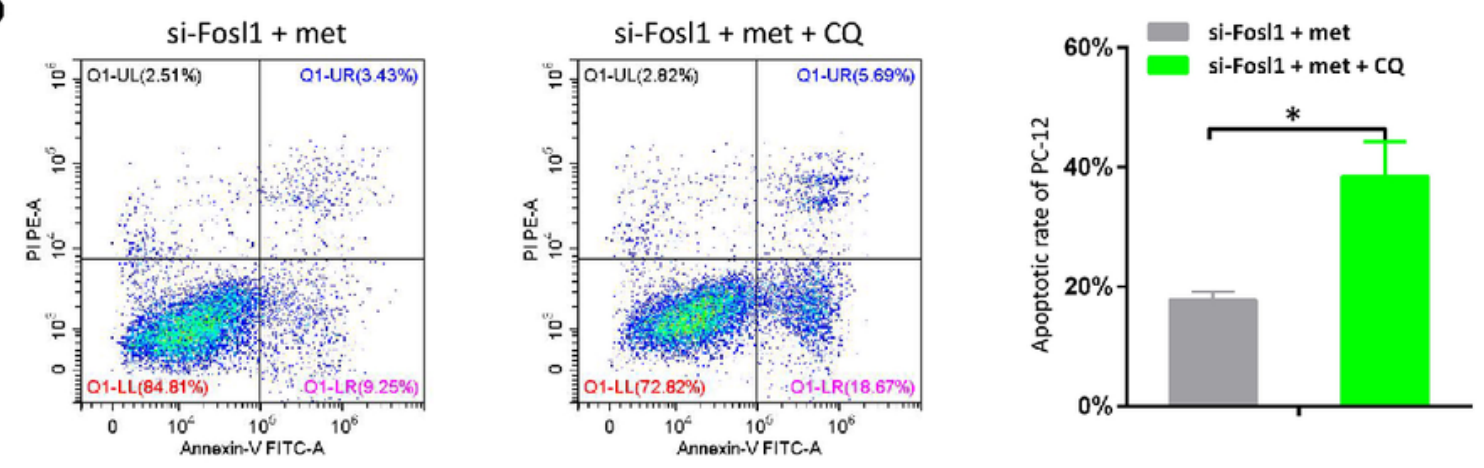


\section{Figure 8}

Inhibition of autophagy neutralized the benefits on the autophagic activity and cell viability after AMPK activation in the injured PC-12 cells with si-Fosl1 treatment but promoted inflammation and cell apoptosis. A: Cell viability was detected using CCK-8 kits in the control group and the CQ group. B: The inflammatory level was checked by ELISA kits. C: The autophagic and apoptotic proteins were visualized by western blotting. D: The cell apoptotic rate was inspected using flow cytometry. All data are presented as the $M \pm S E M\left(n=3\right.$ in each group). ${ }^{*} p<0.05$ vs. si-Fosl $1+$ met group, ${ }^{* \star} p<0.01$ si-Fosl $1+$ met group $(n=3)$ by $T$ test. 

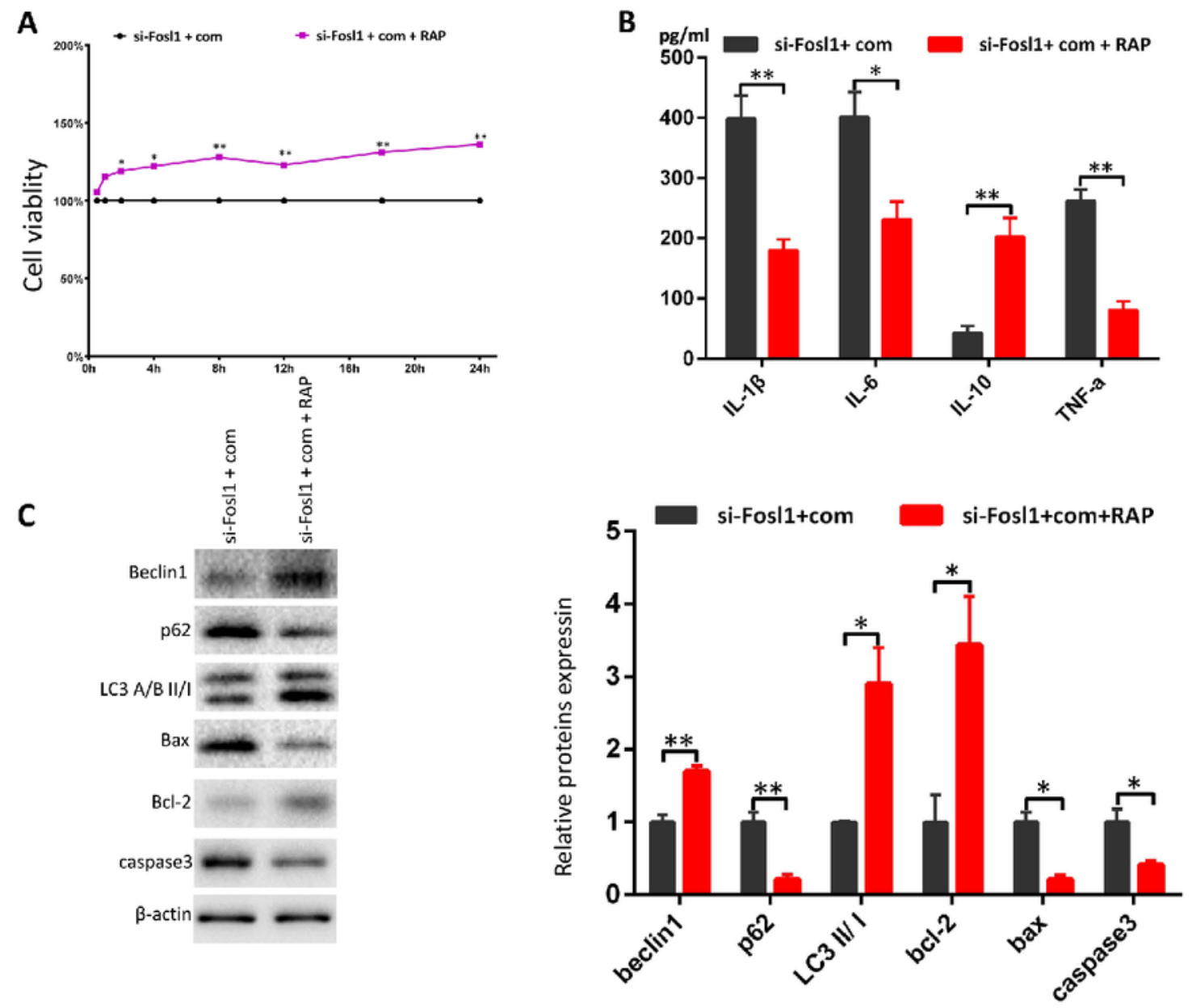

D
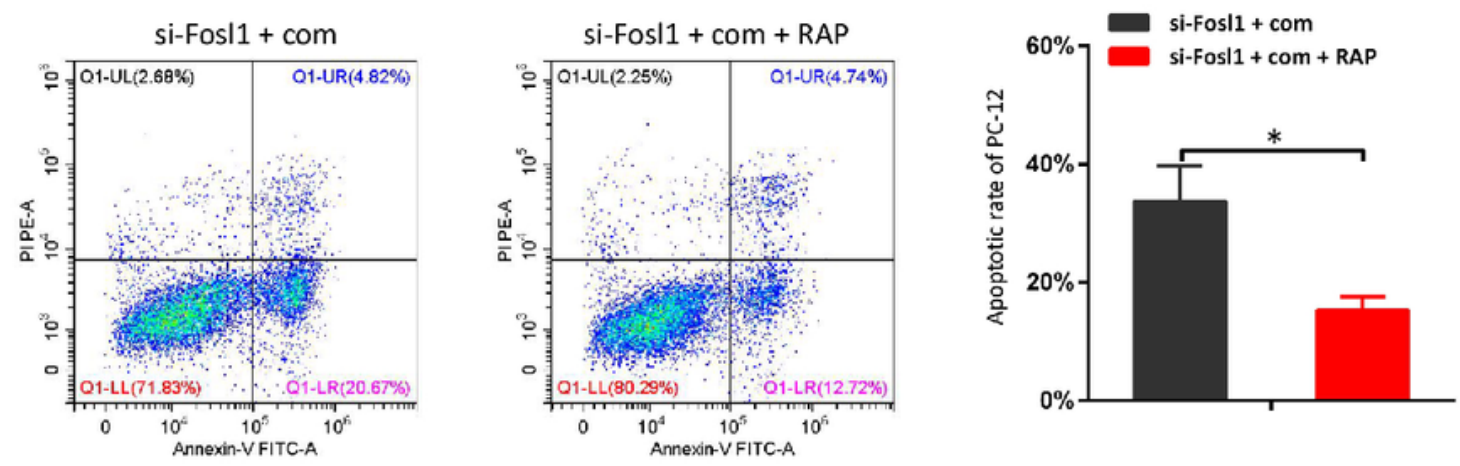

\section{Figure 9}

Activation of autophagy reversed the negative effects on the autophagic activity and cell viability after AMPK inhibition in the injured PC-12 cells with si-Fos 1 treatment and attenuated inflammation and cells apoptosis. A: Cell viability was detected using CCK-8 kits in the control group and the RAP group. B: The inflammatory level was checked by ELISA kits. C: The autophagic and apoptotic proteins were examined by western blotting. D: The cell apoptotic rate was inspected using flow cytometry. All data are presented 
as the $M \pm \operatorname{SEM}\left(\mathrm{n}=3\right.$ in each group). ${ }^{*} \mathrm{p}<0.05$ vs. si-Fosl1 + met group, ${ }^{\star *} \mathrm{p}<0.01$ si-Fosl $1+$ met group $(n=3)$ by $T$ test.
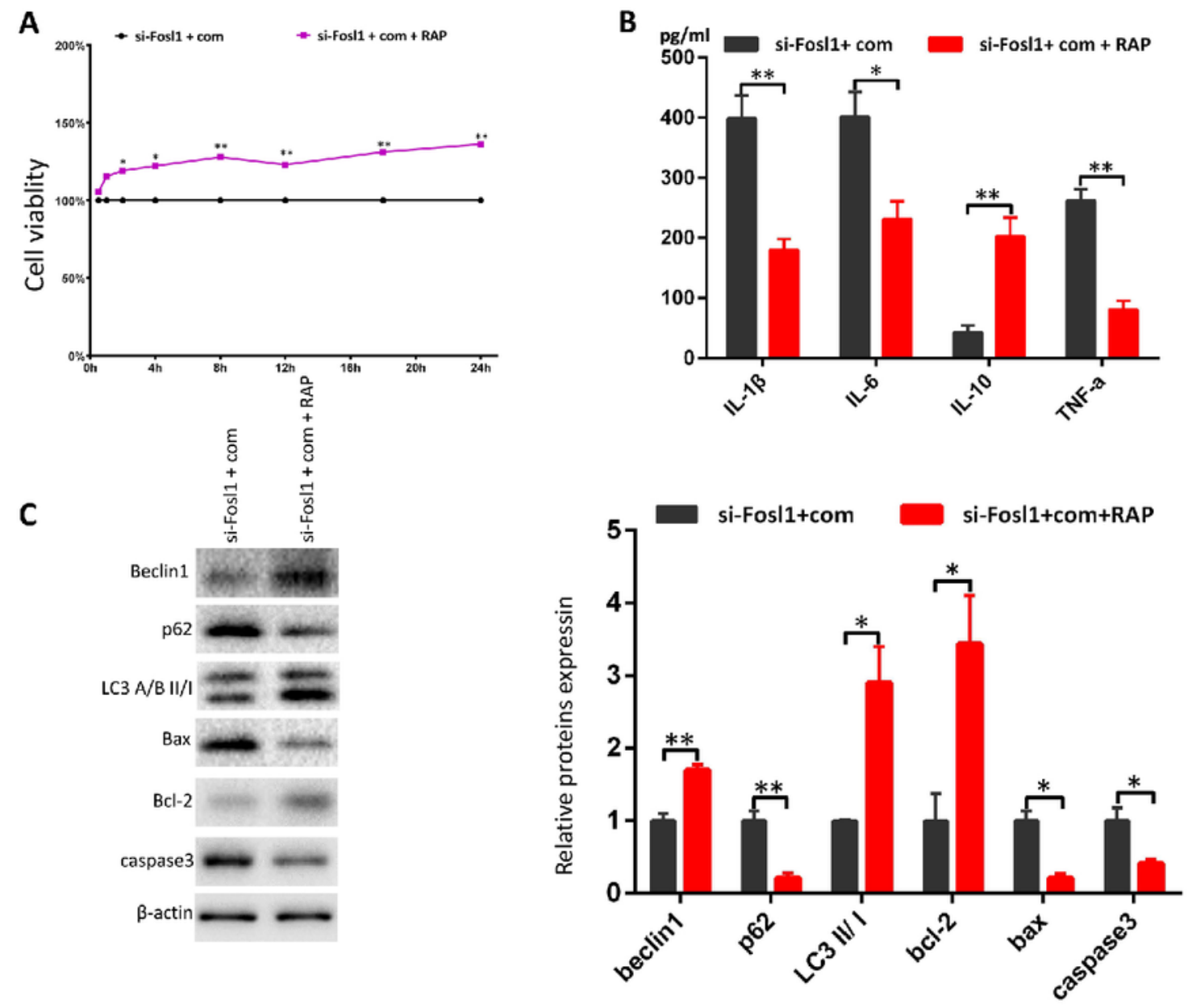

D
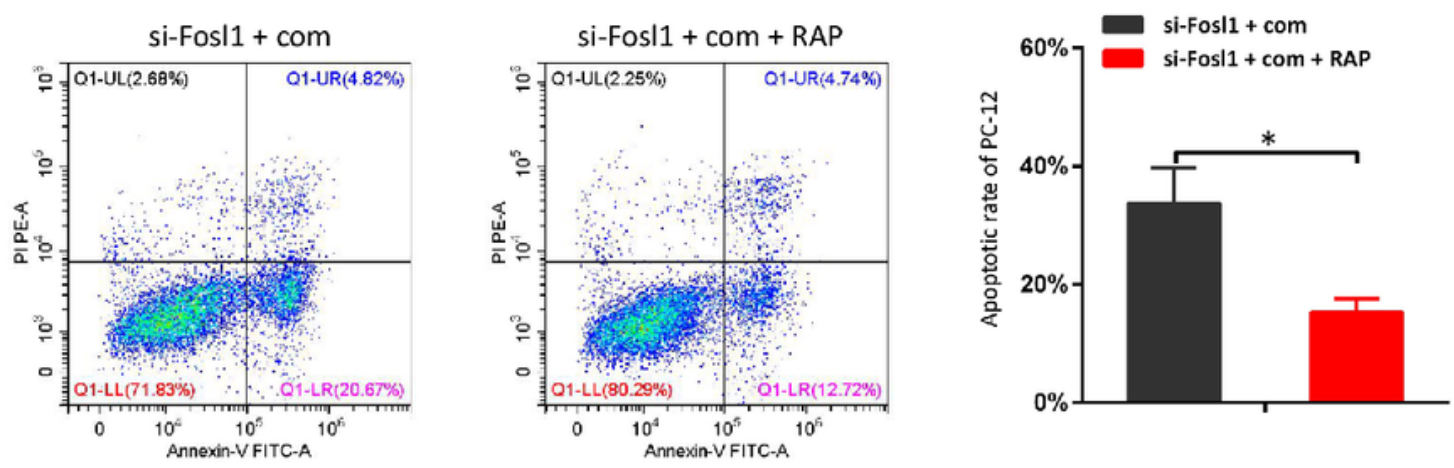

Figure 9

Activation of autophagy reversed the negative effects on the autophagic activity and cell viability after AMPK inhibition in the injured PC-12 cells with si-Fos 1 treatment and attenuated inflammation and cells apoptosis. A: Cell viability was detected using CCK-8 kits in the control group and the RAP group. B: The 
inflammatory level was checked by ELISA kits. C: The autophagic and apoptotic proteins were examined by western blotting. D: The cell apoptotic rate was inspected using flow cytometry. All data are presented as the $M \pm \operatorname{SEM}\left(\mathrm{n}=3\right.$ in each group). ${ }^{*} \mathrm{p}<0.05 \mathrm{vs}$. si-Fosl1 + met group, ${ }^{* \star} \mathrm{p}<0.01 \mathrm{si}-\mathrm{Fos} 11+$ met group $(n=3)$ by $T$ test.
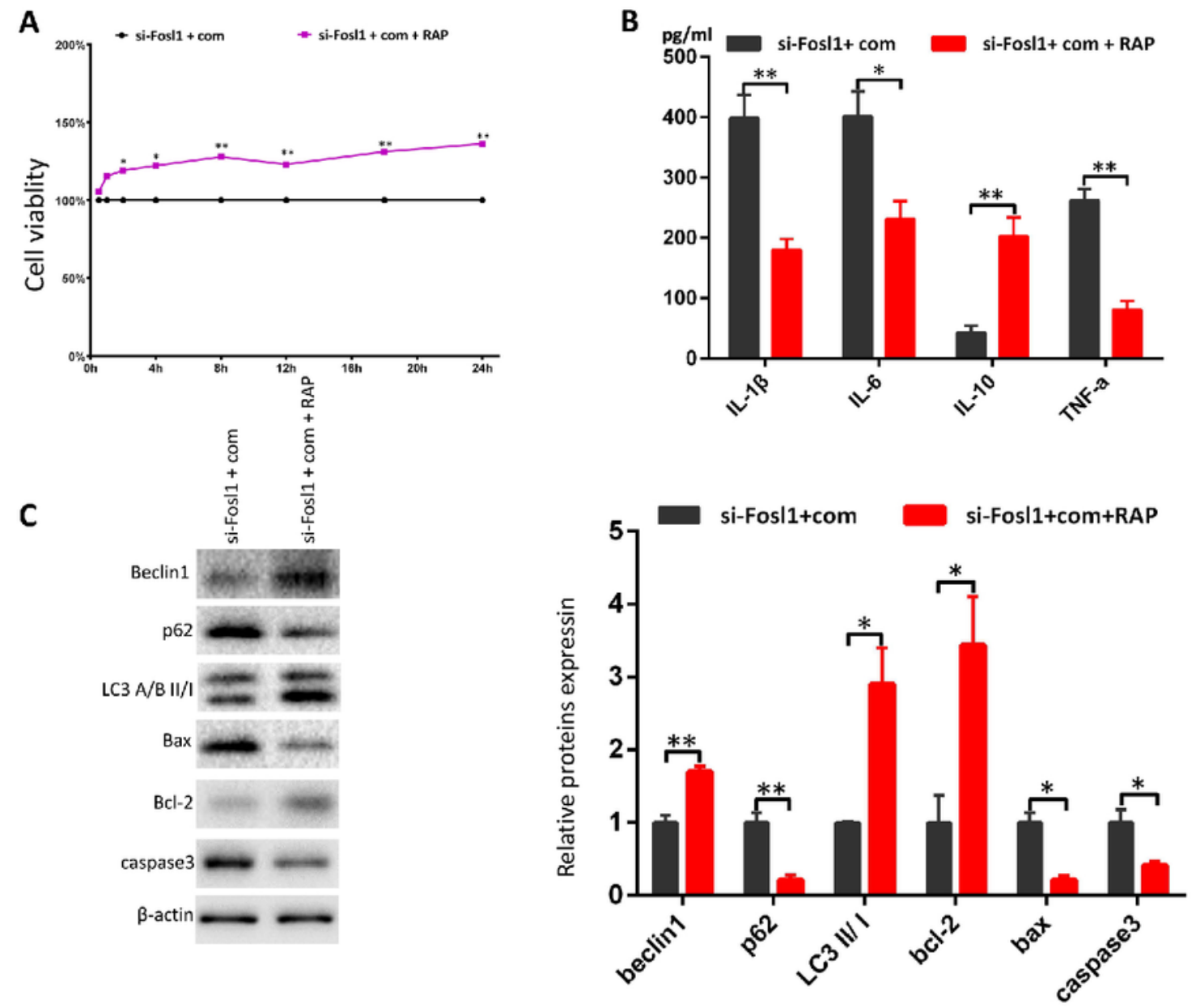

D
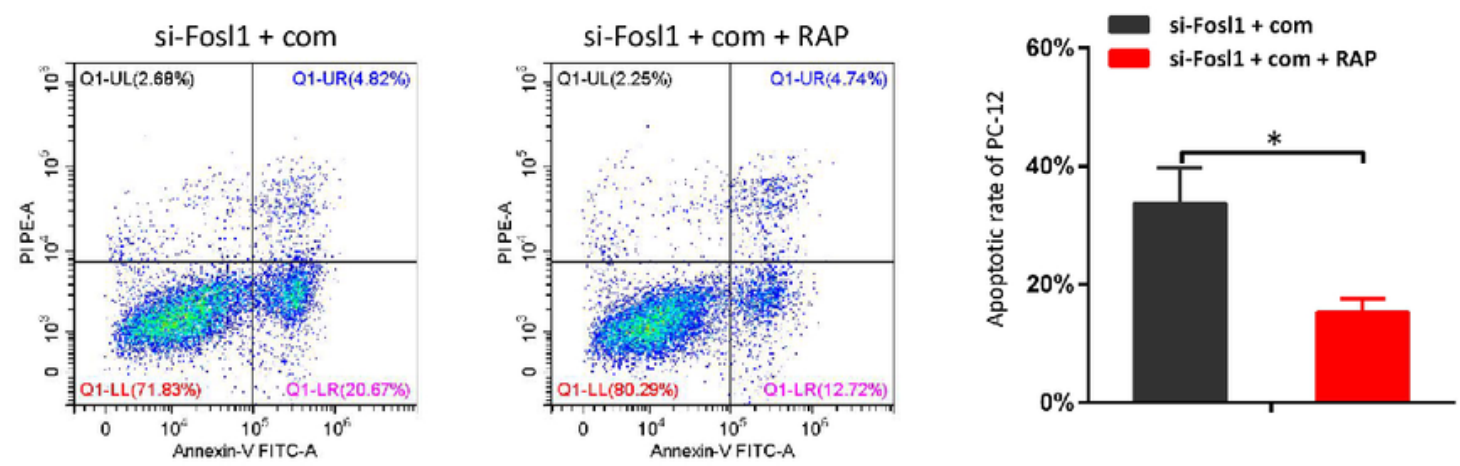

Figure 9 
Activation of autophagy reversed the negative effects on the autophagic activity and cell viability after AMPK inhibition in the injured PC-12 cells with si-Fosl 1 treatment and attenuated inflammation and cells apoptosis. A: Cell viability was detected using CCK-8 kits in the control group and the RAP group. B: The inflammatory level was checked by ELISA kits. C: The autophagic and apoptotic proteins were examined by western blotting. D: The cell apoptotic rate was inspected using flow cytometry. All data are presented as the $M \pm S E M\left(n=3\right.$ in each group). ${ }^{*} p<0.05$ vs. si-Fosl $1+$ met group, ${ }^{*} p<0.01$ si-Fosl $1+$ met group $(n=3)$ by $T$ test.
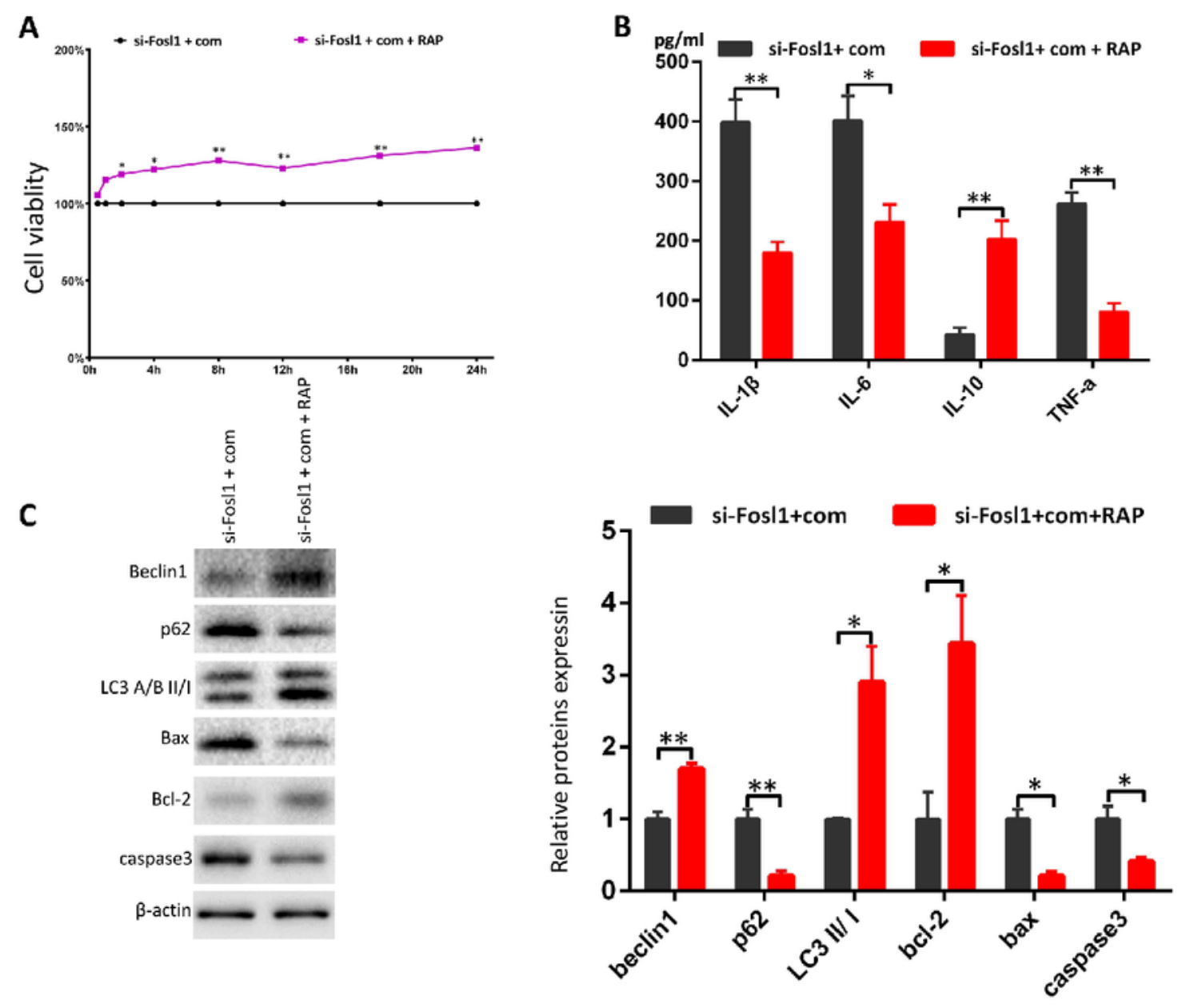

D
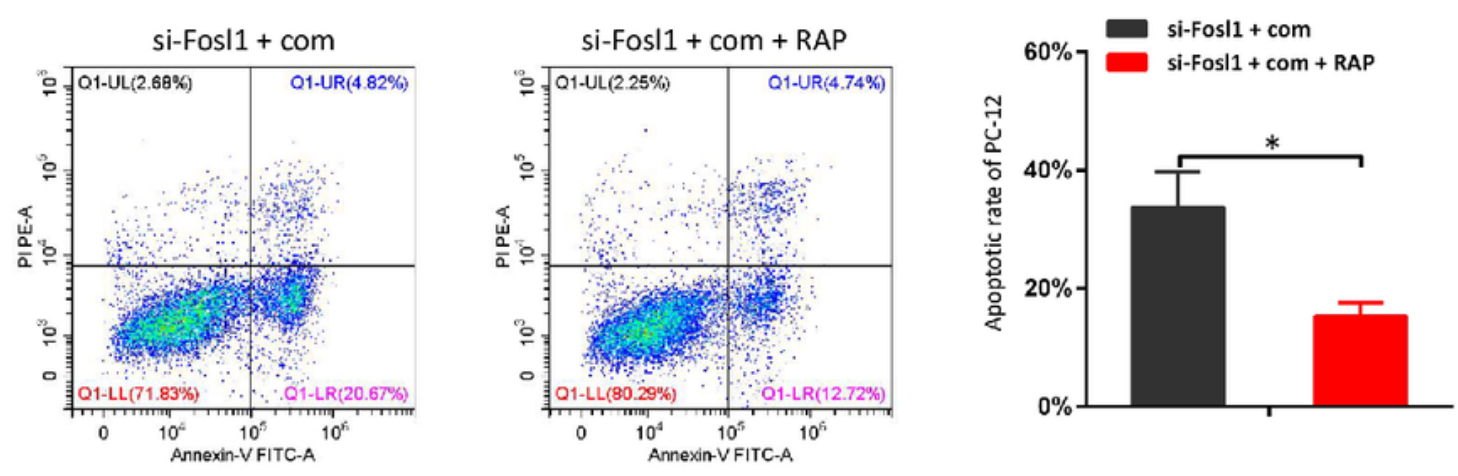


\section{Figure 9}

Activation of autophagy reversed the negative effects on the autophagic activity and cell viability after AMPK inhibition in the injured PC-12 cells with si-Fosl 1 treatment and attenuated inflammation and cells apoptosis. A: Cell viability was detected using CCK-8 kits in the control group and the RAP group. B: The inflammatory level was checked by ELISA kits. C: The autophagic and apoptotic proteins were examined by western blotting. D: The cell apoptotic rate was inspected using flow cytometry. All data are presented as the $M \pm S E M\left(n=3\right.$ in each group). ${ }^{*} p<0.05$ vs. si-Fos $11+$ met group, ${ }^{*} p<0.01$ si-Fosl $1+$ met group $(n=3)$ by $T$ test.
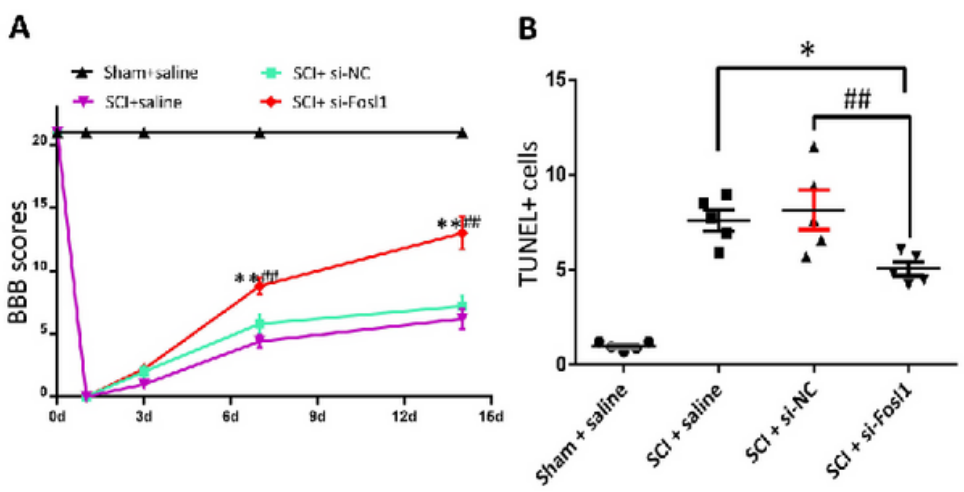

C

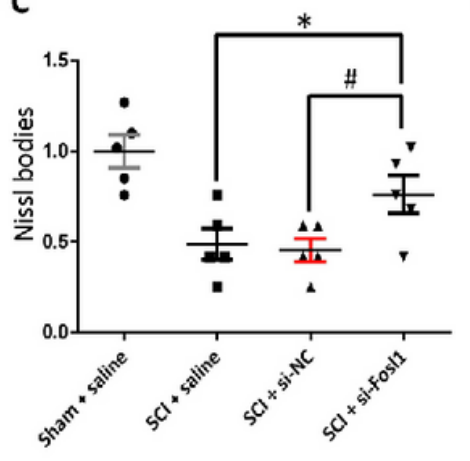

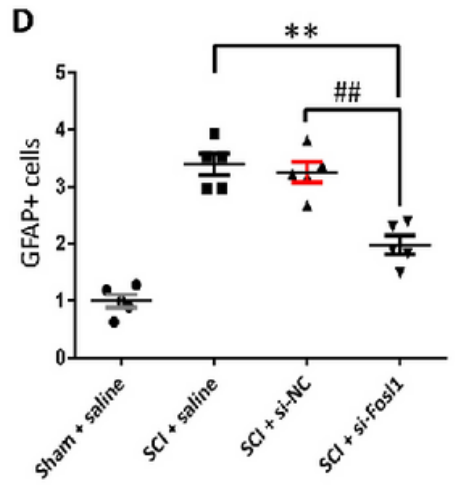

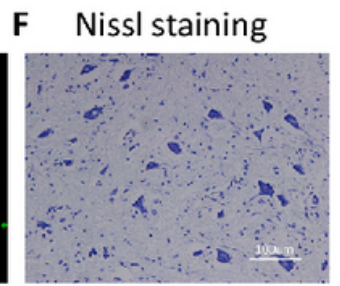

G
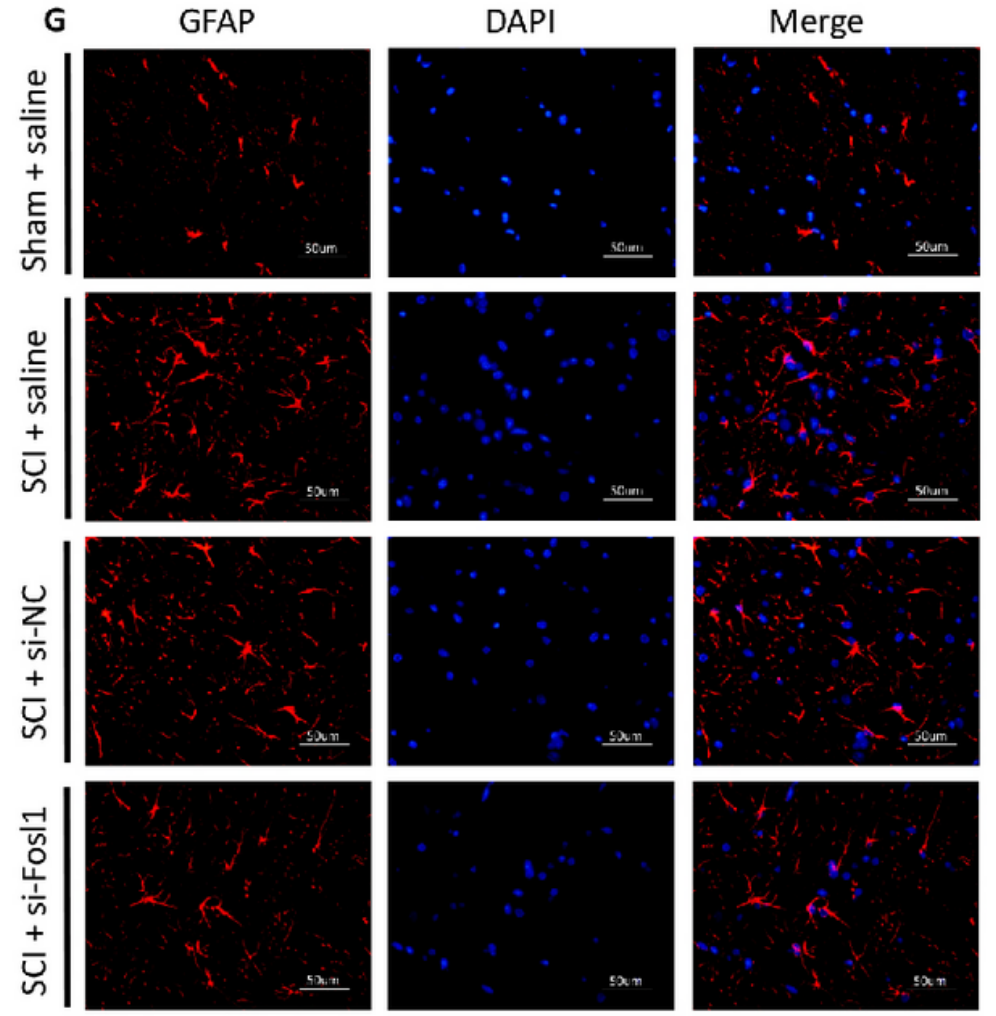

\section{Figure 10}

Fosl1 knockdown improve neurological function and decrease cell apoptosis, the loss of motor neurons and inflammatory activity. A: The BBB scores of four groups at each time point. B-D: The histograms of TUNEL+ cells, Nissl bodies and GFAP+ cells. E: TUNEL staining at $14 \mathrm{~d}$ (magnification $\times 400)$. F: Nissl 
staining at $14 \mathrm{~d}$ (magnification $\times 200)$. G: The Immunofluorescence staining of GFAP at $14 \mathrm{~d}$ (magnification $\times 400)$. All data are presented as the $M \pm S E M\left(n=5\right.$ in each group). ${ }^{*} p<0.05 \mathrm{vs}$. $S C l+$ saline group, ${ }^{\star *} p<0.01 \mathrm{vs.} \mathrm{SCl}+$ saline group $(n=5)$. \#p<0.05 vs. $\mathrm{SCl}+\operatorname{si}-\mathrm{NC}$ group, $\# \# p<0.01 \mathrm{vs}$. $\mathrm{SCl}+\mathrm{si}-$ NC group $(n=5)$ by ANOVA.
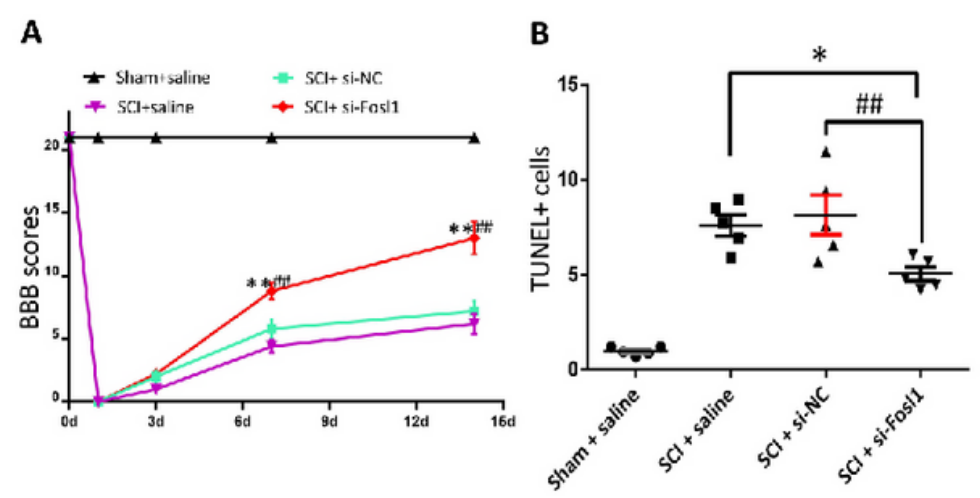

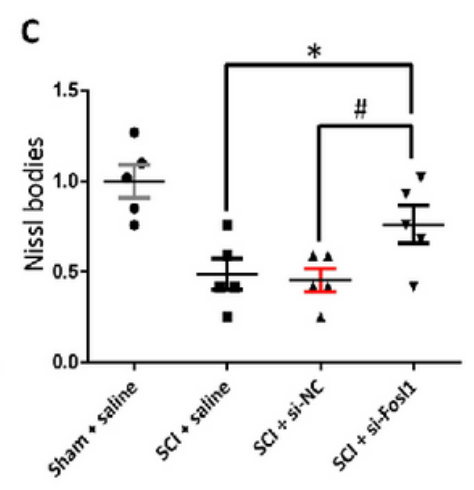

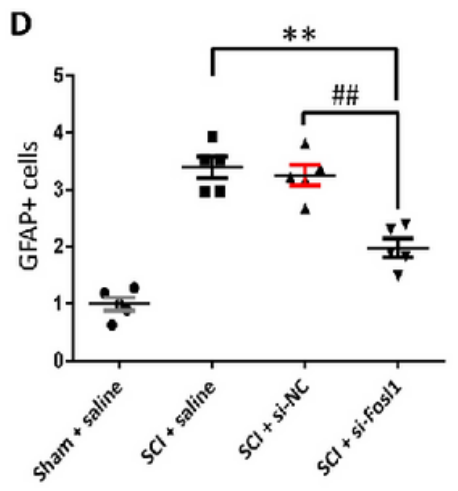
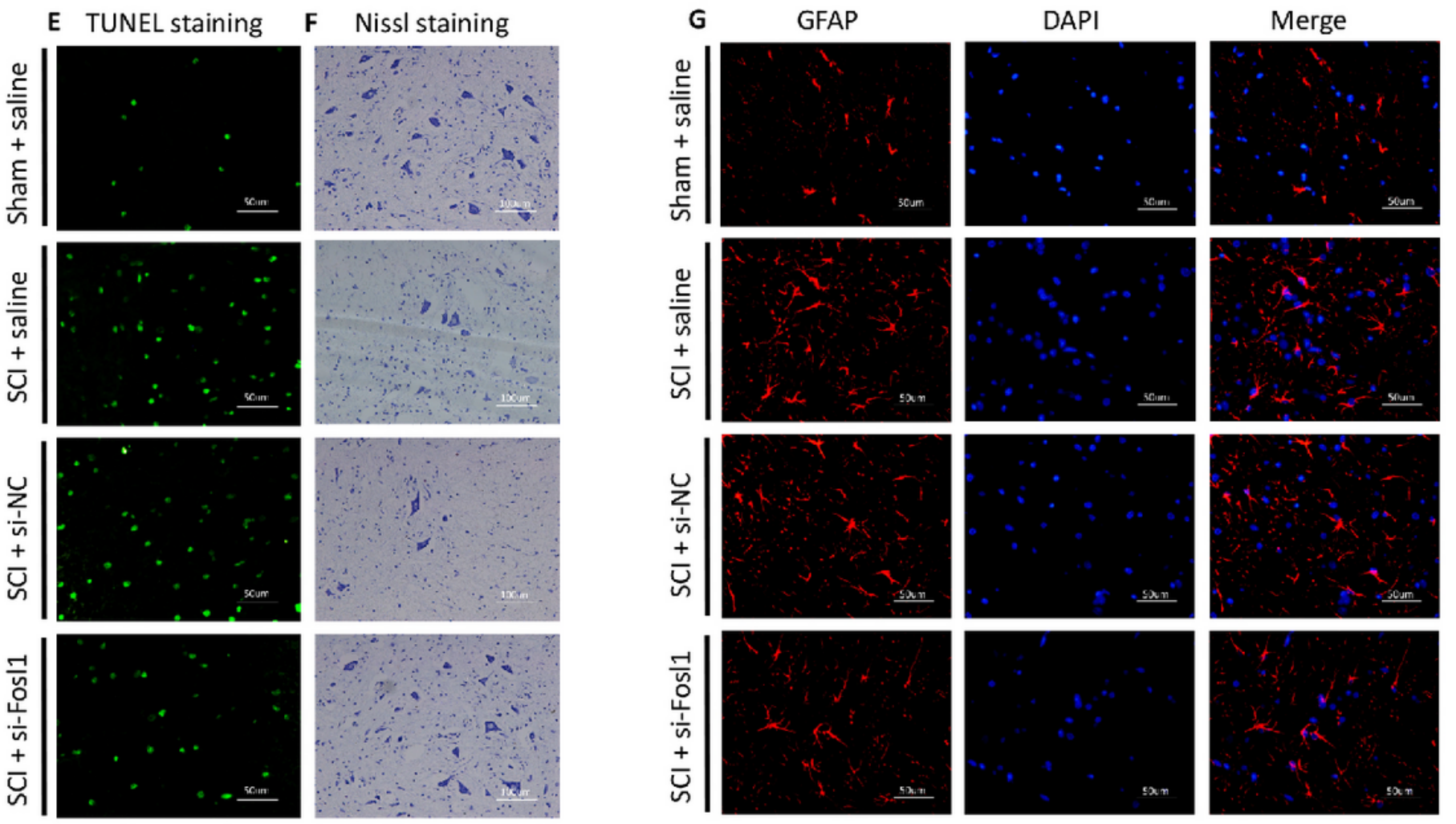

\section{Figure 10}

Fosl1 knockdown improve neurological function and decrease cell apoptosis, the loss of motor neurons and inflammatory activity. A: The BBB scores of four groups at each time point. B-D: The histograms of TUNEL+ cells, Nissl bodies and GFAP+ cells. E: TUNEL staining at $14 \mathrm{~d}$ (magnification $\times 400)$. F: Nissl staining at $14 \mathrm{~d}$ (magnification $\times 200$ ). G: The Immunofluorescence staining of GFAP at $14 \mathrm{~d}$ (magnification $\times 400)$. All data are presented as the $M \pm S E M\left(n=5\right.$ in each group). ${ }^{*} p<0.05 \mathrm{vs}$. $S C l+$

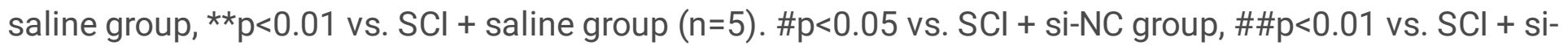
NC group $(n=5)$ by ANOVA. 

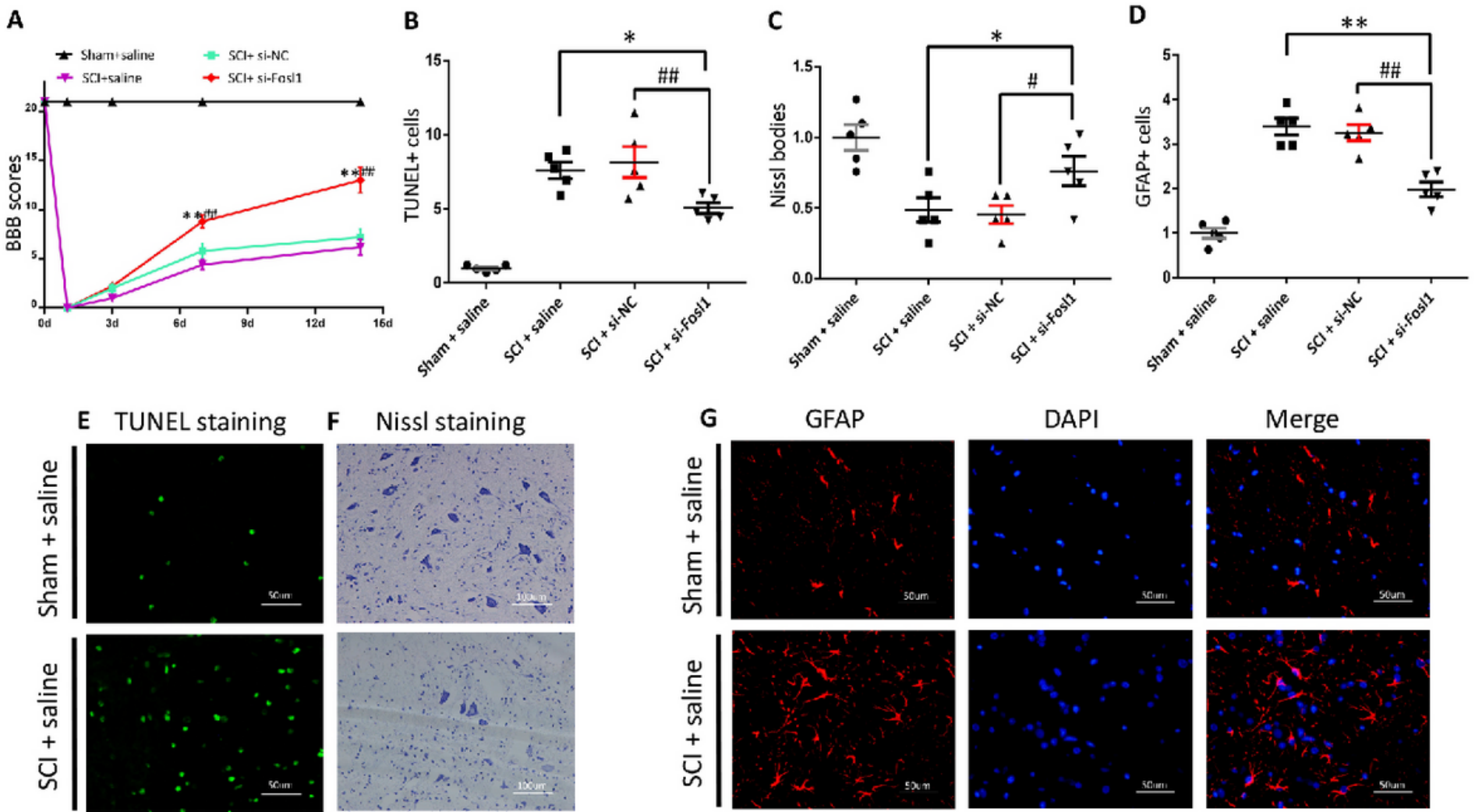

Merge
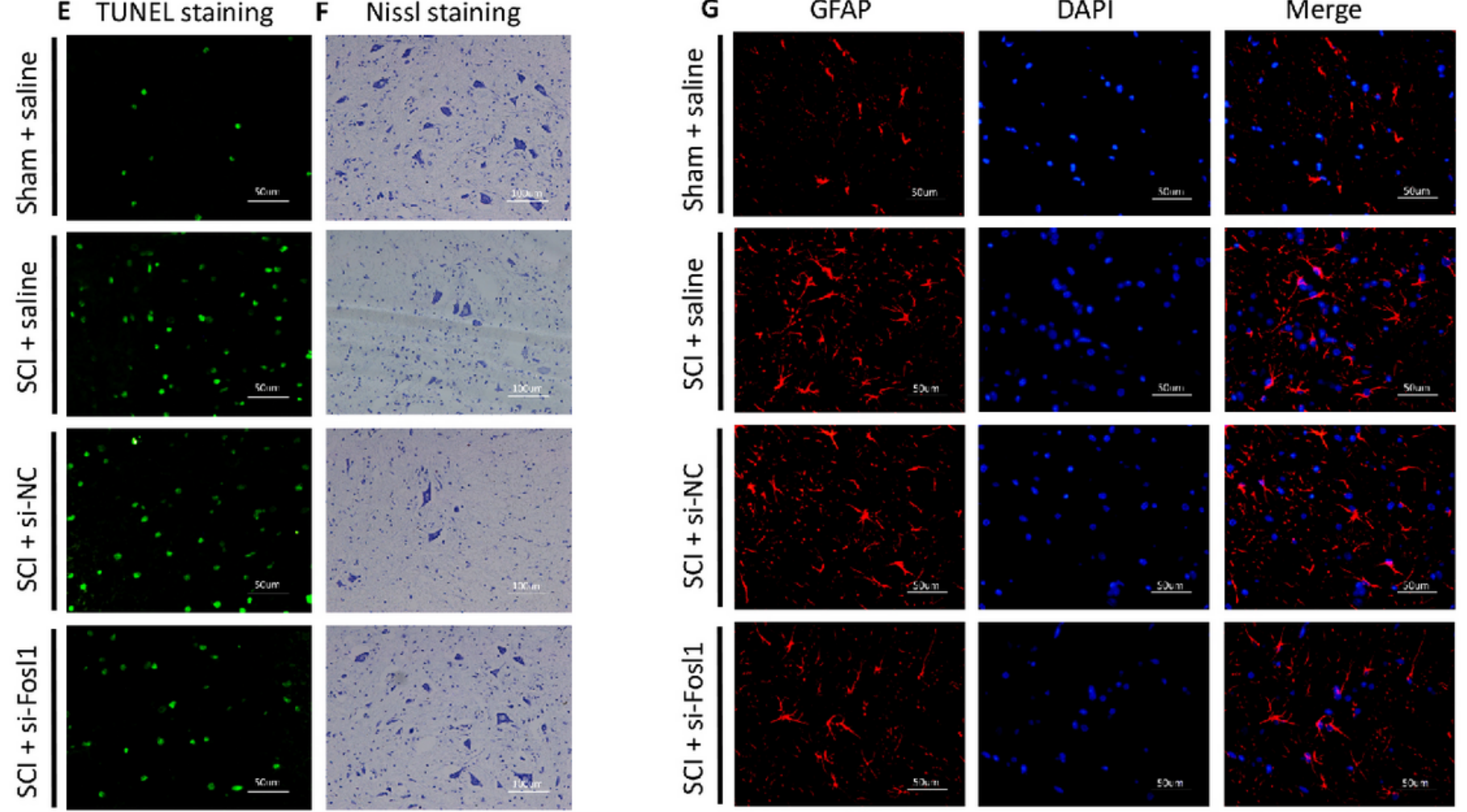

\section{Figure 10}

Fosl1 knockdown improve neurological function and decrease cell apoptosis, the loss of motor neurons and inflammatory activity. A: The BBB scores of four groups at each time point. B-D: The histograms of TUNEL+ cells, Nissl bodies and GFAP+ cells. E: TUNEL staining at $14 \mathrm{~d}$ (magnification $\times 400)$. F: Nissl staining at $14 \mathrm{~d}$ (magnification $\times 200$ ). G: The Immunofluorescence staining of GFAP at $14 \mathrm{~d}$ (magnification $\times 400)$. All data are presented as the $M \pm S E M\left(n=5\right.$ in each group). ${ }^{*} p<0.05$ vs. $S C l+$ saline group, ${ }^{\star *} p<0.01$ vs. $S C l+$ saline group $(n=5)$. \#p<0.05 vs. $S C l+s i-N C$ group, $\# \# p<0.01 v s . S C l+s i-$ NC group $(n=5)$ by ANOVA. 

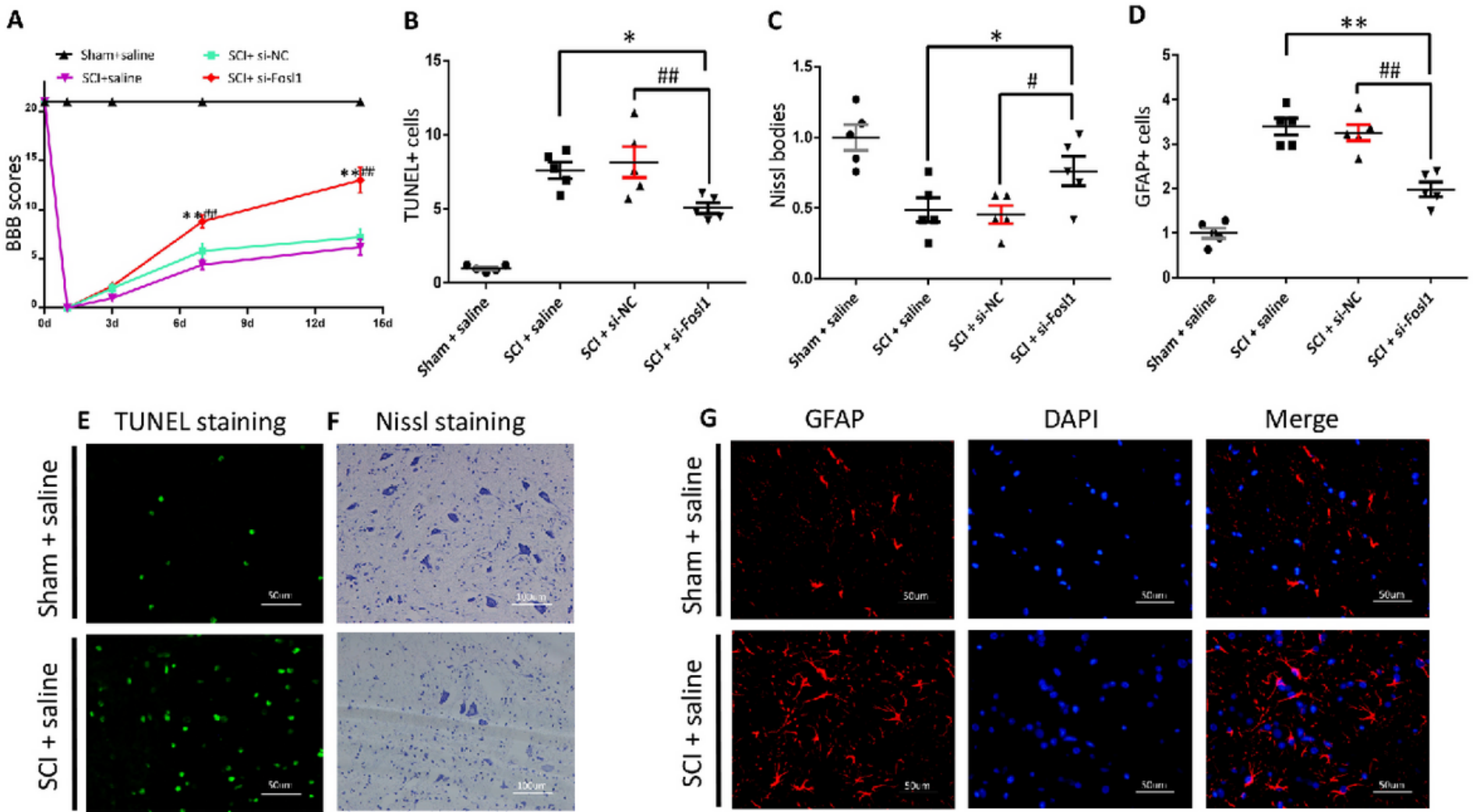

Merge
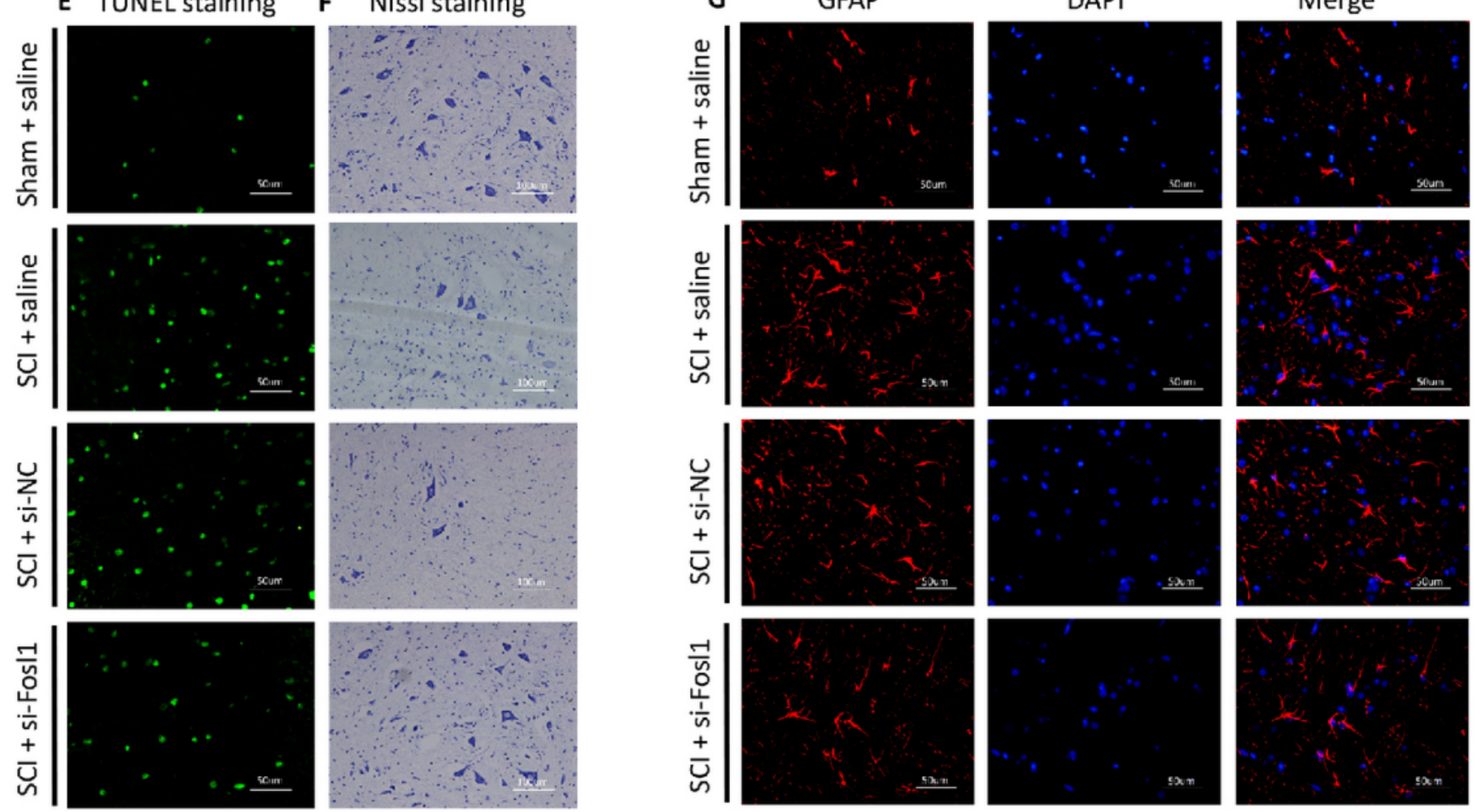

\section{Figure 10}

Fosl1 knockdown improve neurological function and decrease cell apoptosis, the loss of motor neurons and inflammatory activity. A: The BBB scores of four groups at each time point. B-D: The histograms of TUNEL+ cells, Nissl bodies and GFAP+ cells. E: TUNEL staining at $14 \mathrm{~d}$ (magnification $\times 400)$. F: Nissl staining at $14 \mathrm{~d}$ (magnification $\times 200$ ). G: The Immunofluorescence staining of GFAP at $14 \mathrm{~d}$ (magnification $\times 400)$. All data are presented as the $M \pm S E M\left(n=5\right.$ in each group). ${ }^{*} p<0.05 \mathrm{vs}$. $S C l+$ saline group, ${ }^{* \star} p<0.01 \mathrm{vs}$. $S C l+$ saline group $(n=5)$. $\# p<0.05$ vs. $S C l+s i-N C$ group, $\# \# p<0.01 \mathrm{vs}$. $S C l+s i-$ NC group $(n=5)$ by ANOVA. 

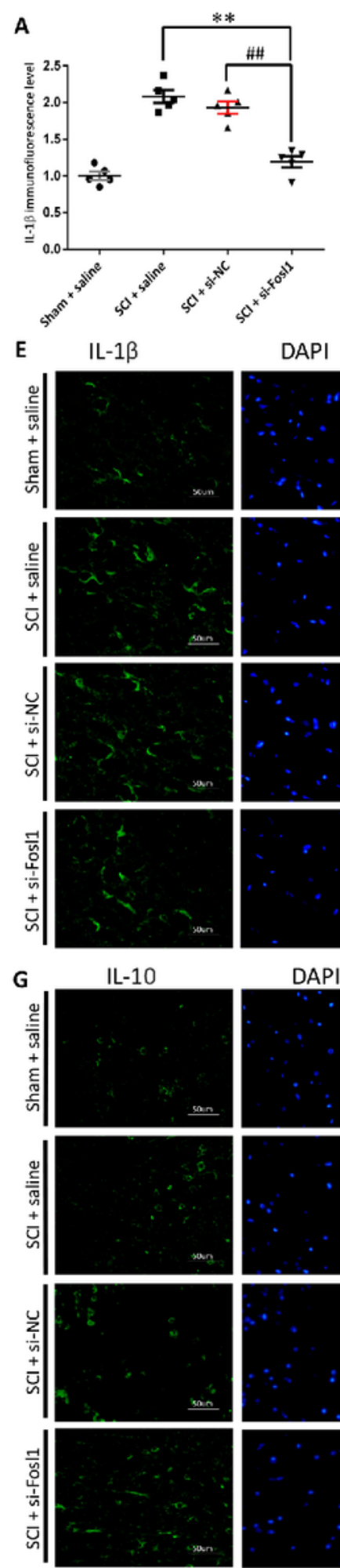

DAPI

DAPI
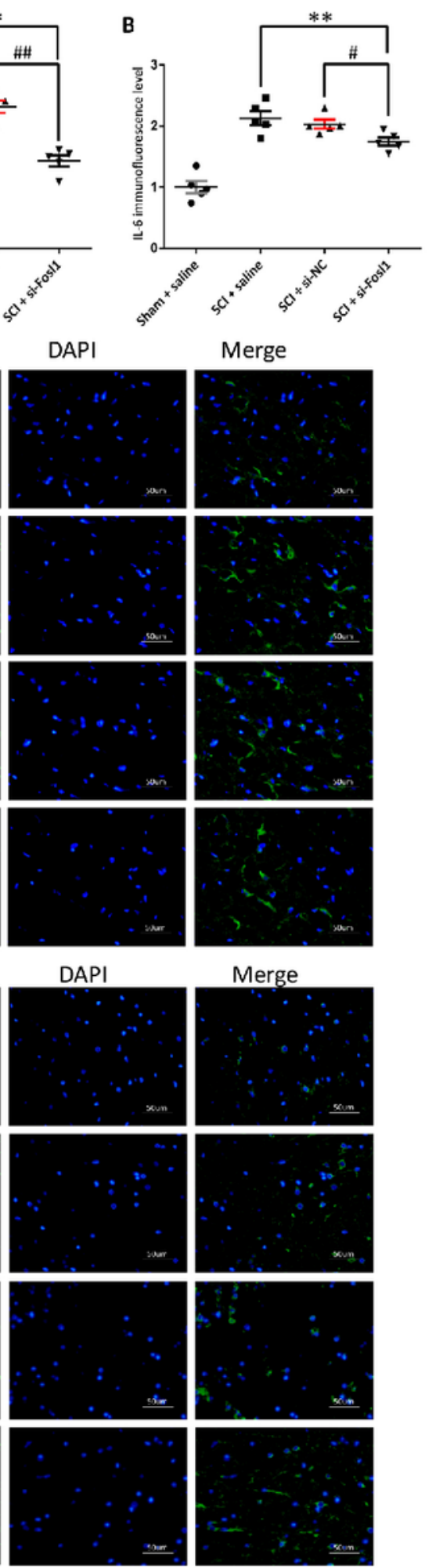

Merge
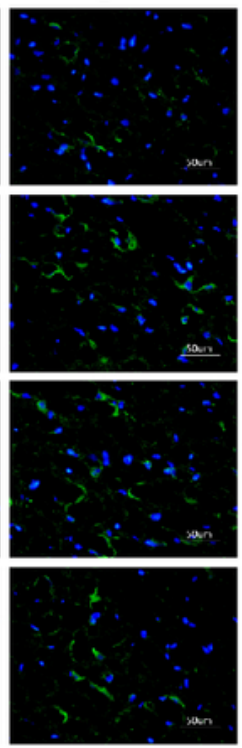

Merge
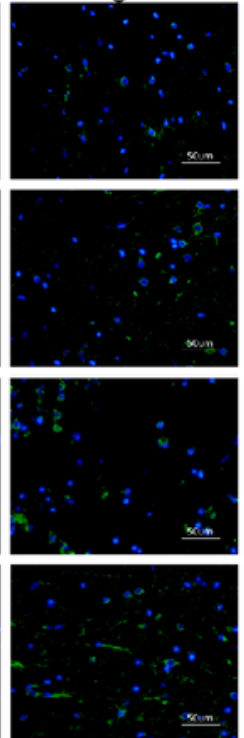
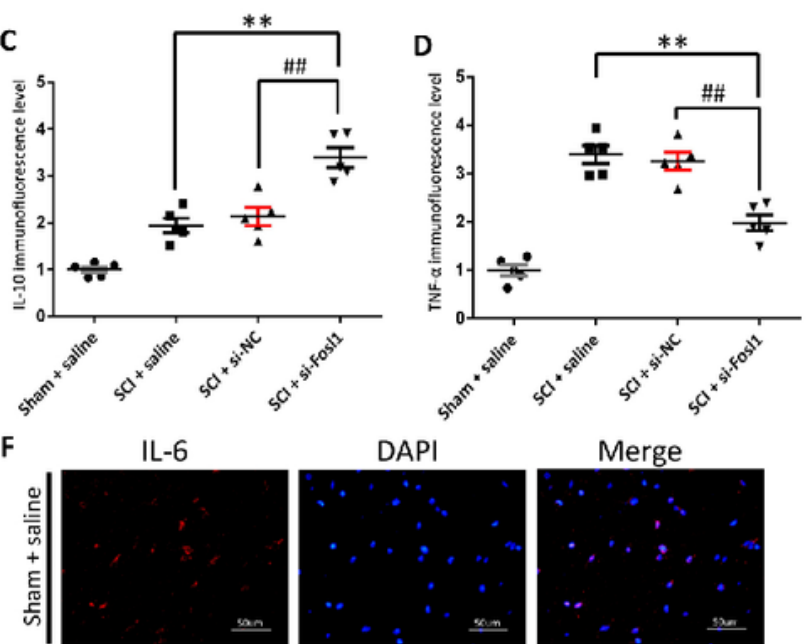

Merge
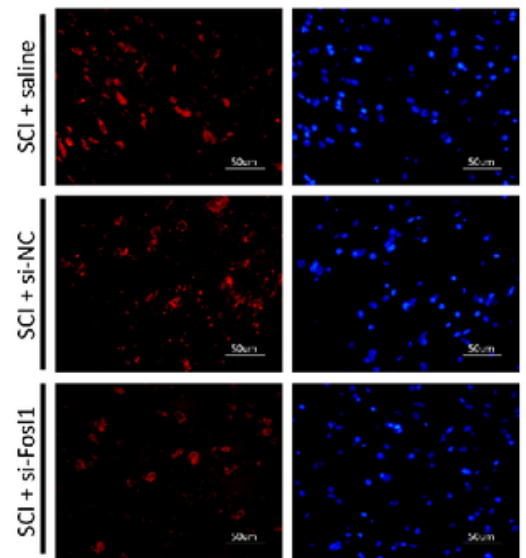

DAPI
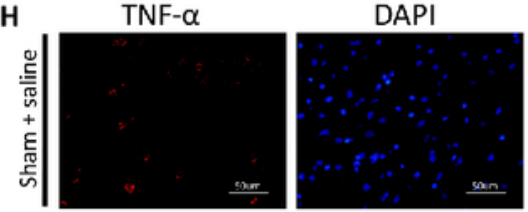

Merge
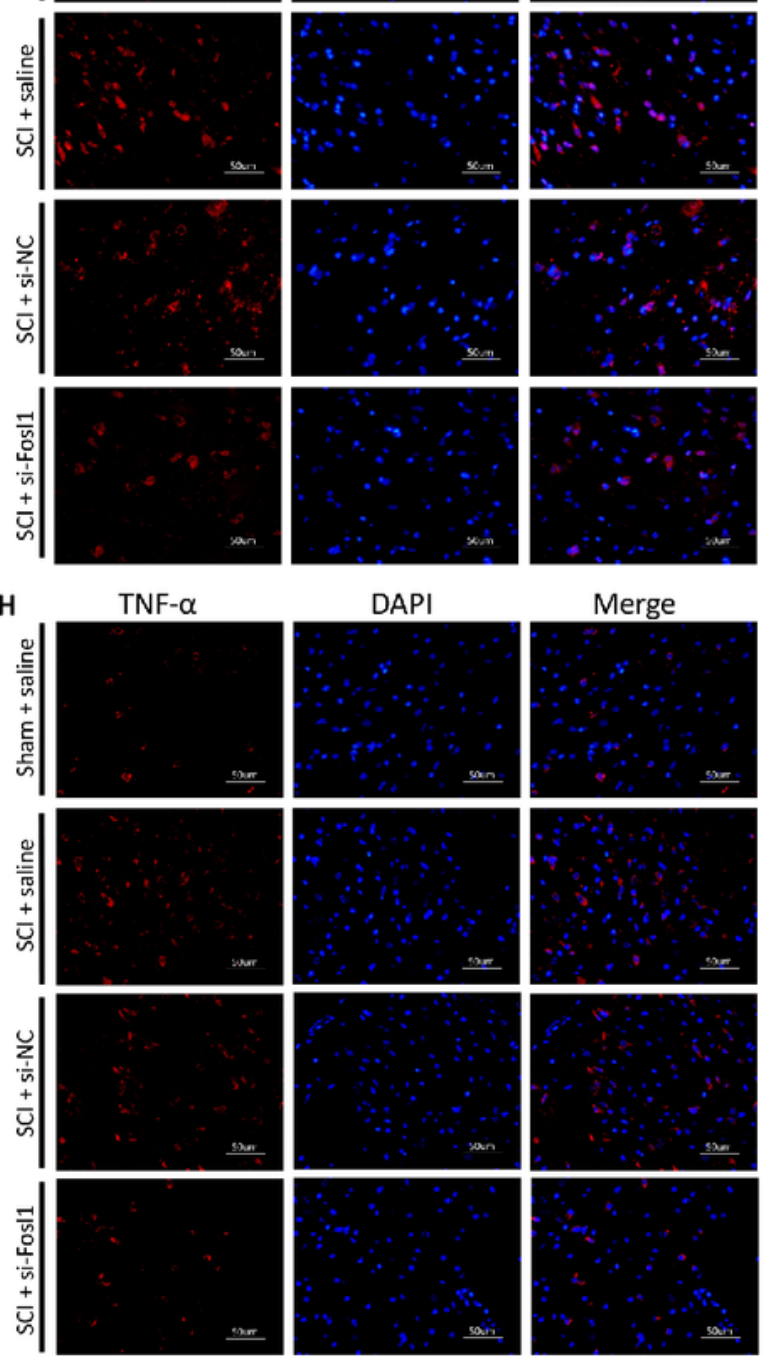

\section{Figure 11}

Fosl1 knockdown downregulated inflammatory cytokines and enhanced anti-ininflammatory cytokine. AD: The histograms of IL-1 $\beta$, IL-6, IL-10 and TNF- $\alpha$ expressions. E: The immunofluorescence staining of IL$1 \beta, I L-6, I L-10$ and TNF- $\alpha$ at $14 d$ (magnification $\times 400)$. All data are presented as the $M \pm S E M(n=5$ in each group). ${ }^{\star} p<0.05 \mathrm{vs}$. $\mathrm{SCl}+$ saline group, ${ }^{\star *} \mathrm{p}<0.01 \mathrm{vs}$. $\mathrm{SCl}+$ saline group $(\mathrm{n}=5) . \# \mathrm{p}<0.05 \mathrm{vs}$. $\mathrm{SCl}+\mathrm{si}-$ NC group, \#\#p<0.01 vs. SCI + si-NC group $(n=5)$ by ANOVA. 

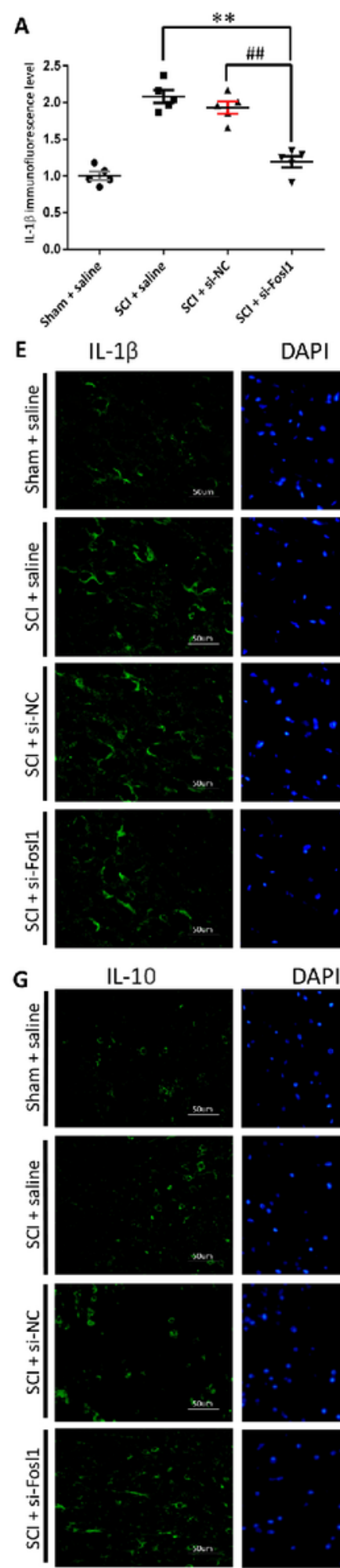

DAPI

DAPI
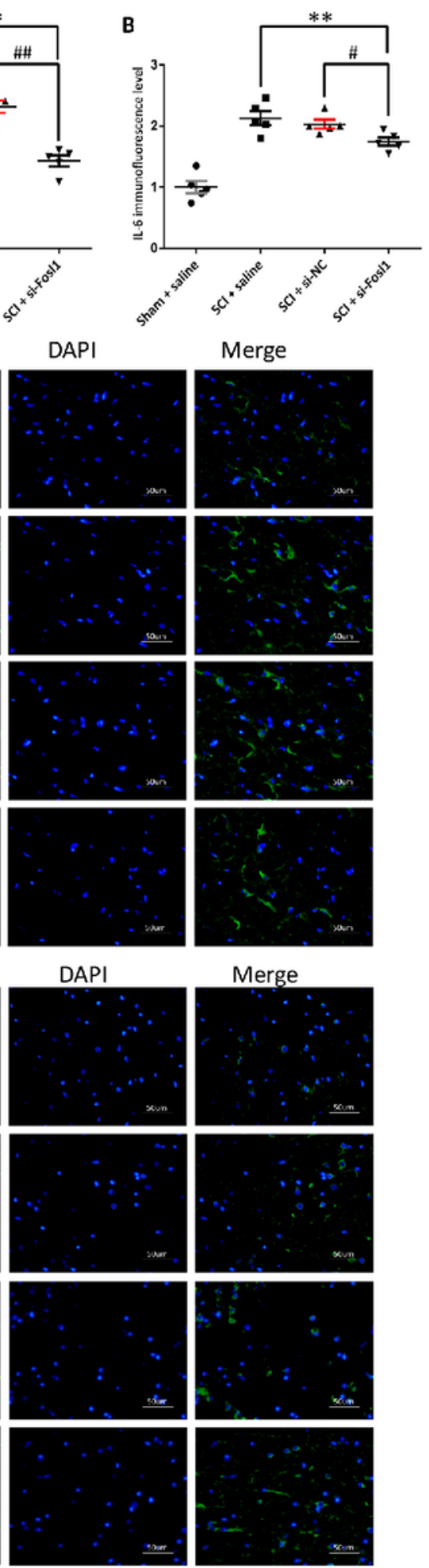

Merge
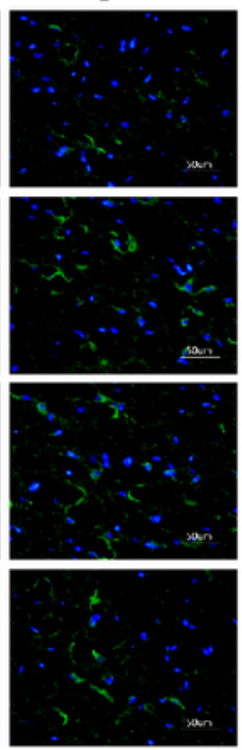

Merge
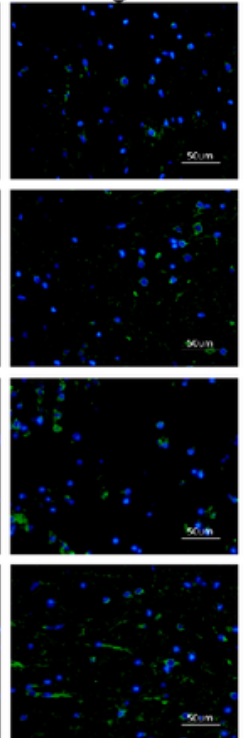
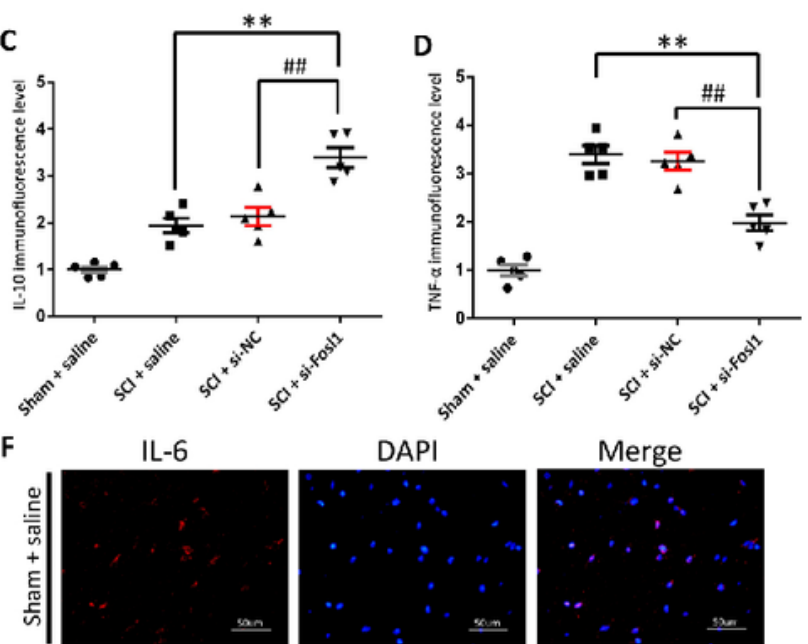

Merge
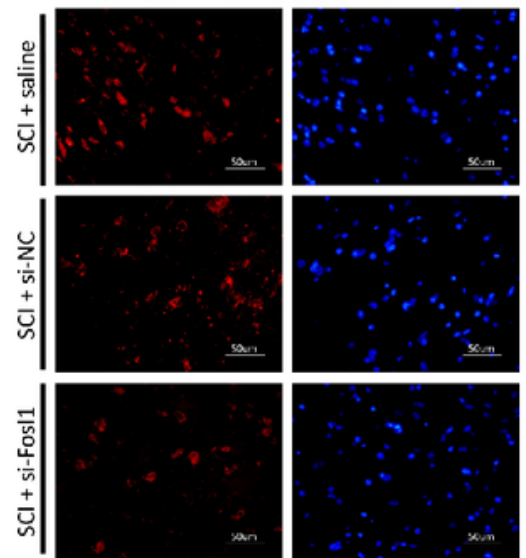

DAPI
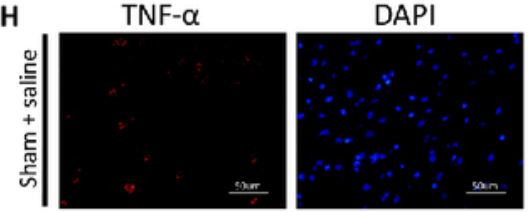

Merge
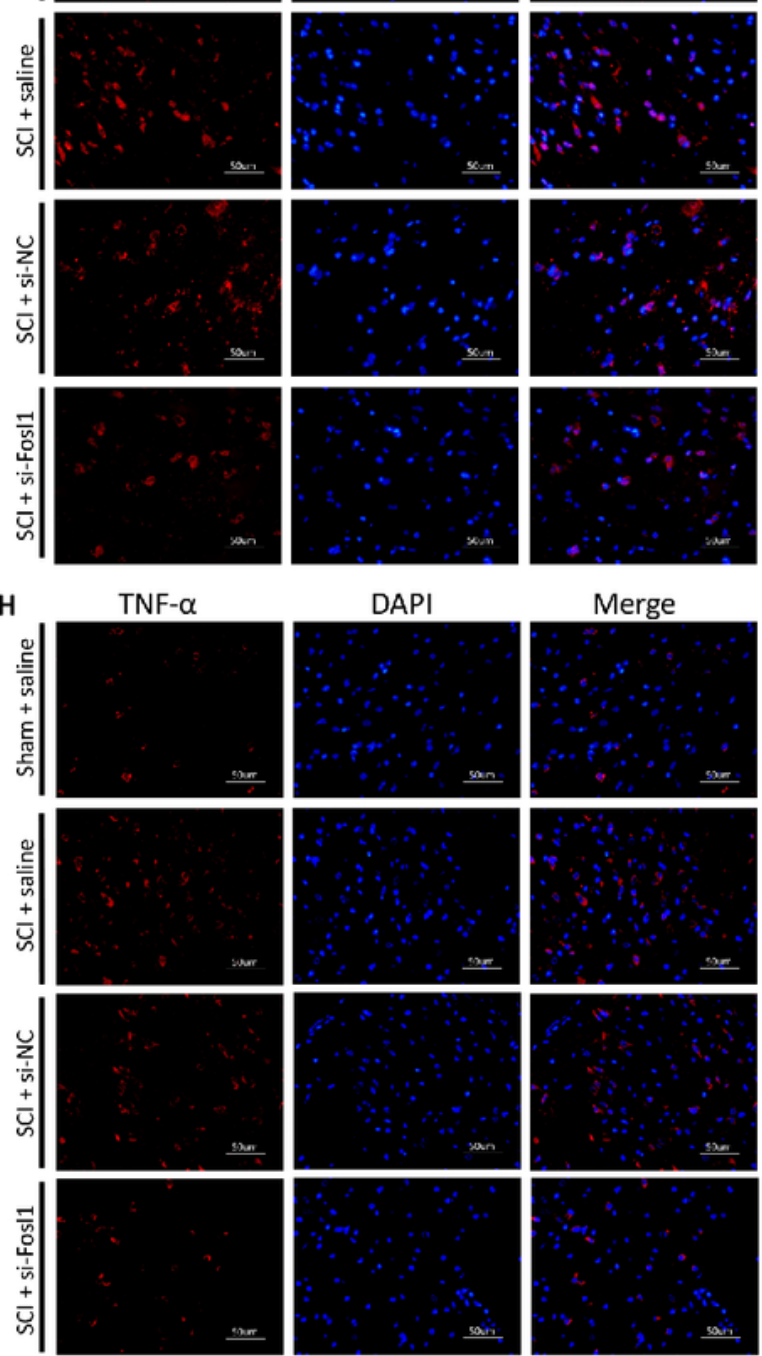

\section{Figure 11}

Fosl1 knockdown downregulated inflammatory cytokines and enhanced anti-ininflammatory cytokine. AD: The histograms of IL-1 $\beta$, IL-6, IL-10 and TNF- $\alpha$ expressions. E: The immunofluorescence staining of IL$1 \beta, I L-6, I L-10$ and TNF- $\alpha$ at $14 d$ (magnification $\times 400)$. All data are presented as the $M \pm S E M(n=5$ in each group). ${ }^{\star} p<0.05 \mathrm{vs}$. $\mathrm{SCl}+$ saline group, ${ }^{\star *} \mathrm{p}<0.01 \mathrm{vs}$. $\mathrm{SCl}+$ saline group $(\mathrm{n}=5) . \# \mathrm{p}<0.05 \mathrm{vs}$. $\mathrm{SCl}+\mathrm{si}-$ NC group, \#\#p<0.01 vs. SCI + si-NC group $(n=5)$ by ANOVA. 

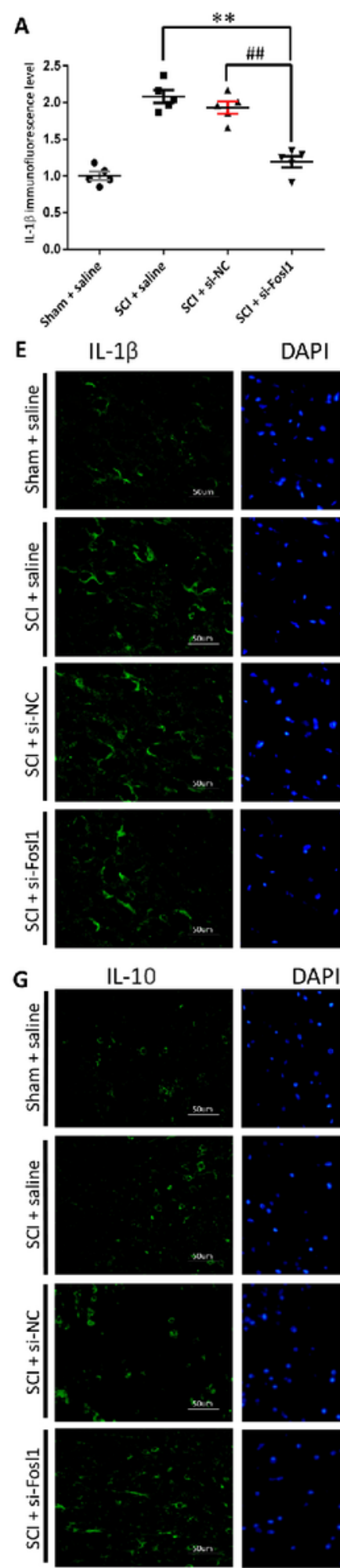

DAPI

DAPI
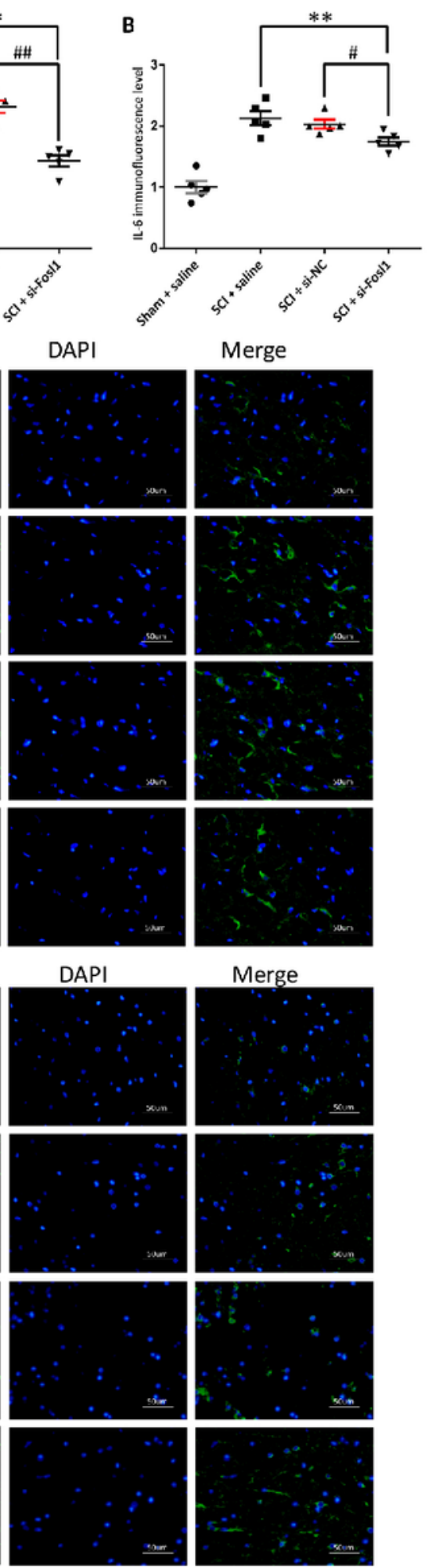

Merge
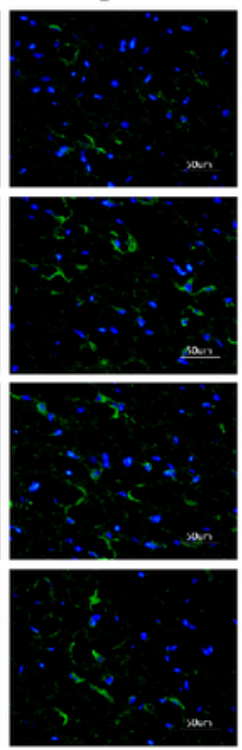

Merge
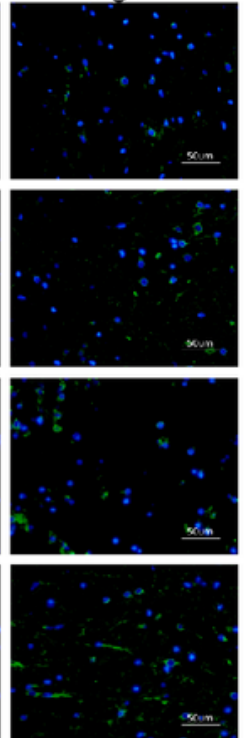
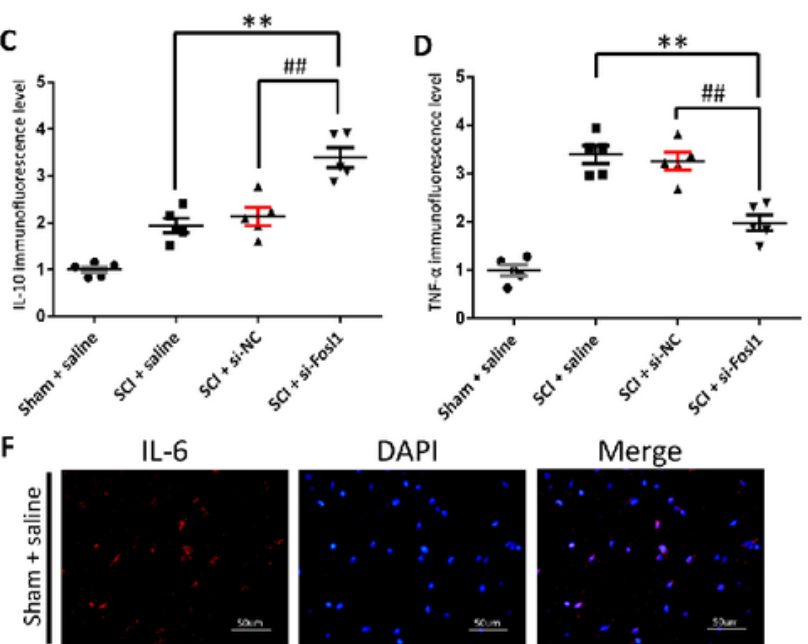

Merge
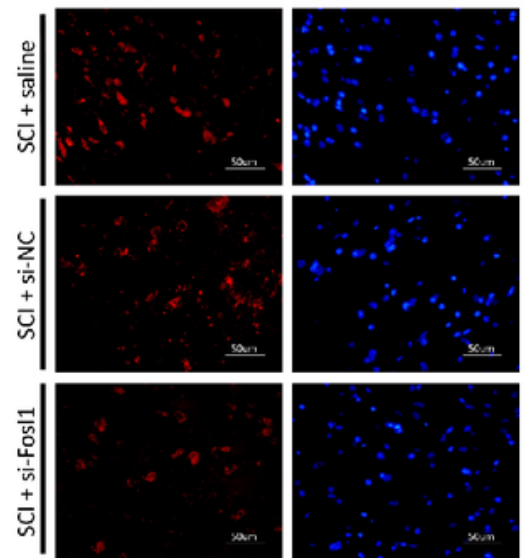

DAPI
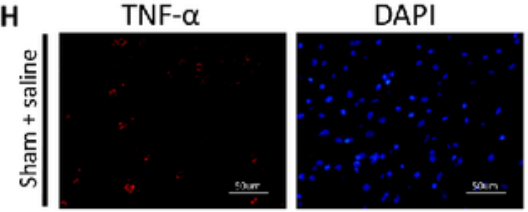

Merge
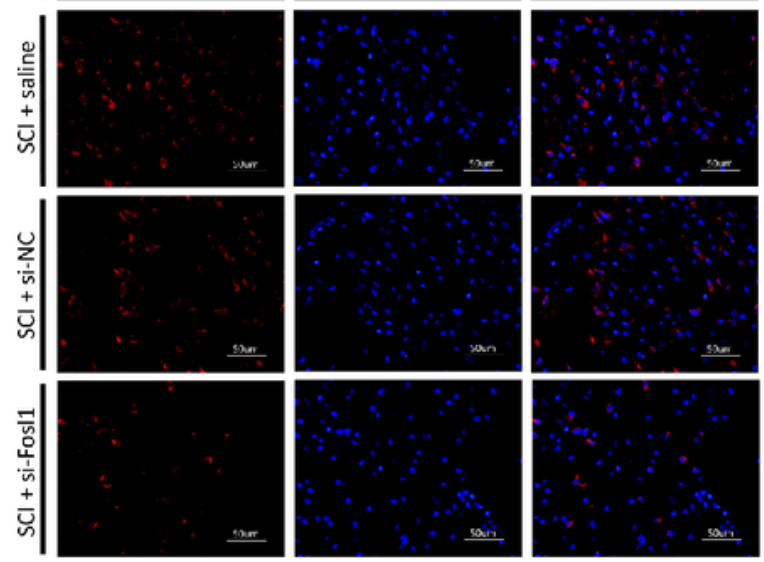

\section{Figure 11}

Fosl1 knockdown downregulated inflammatory cytokines and enhanced anti-ininflammatory cytokine. AD: The histograms of IL-1 $\beta$, IL-6, IL-10 and TNF- $\alpha$ expressions. E: The immunofluorescence staining of IL$1 \beta, I L-6, I L-10$ and TNF- $\alpha$ at $14 d$ (magnification $\times 400)$. All data are presented as the $M \pm S E M(n=5$ in each group). ${ }^{*} p<0.05 \mathrm{vs} . \mathrm{SCl}+$ saline group, ${ }^{\star *} p<0.01 \mathrm{vs}$. $\mathrm{SCl}+$ saline group $(n=5) . \# p<0.05 \mathrm{vs}$. $\mathrm{SCl}+\mathrm{si}-$ NC group, \#\#p<0.01 vs. SCI + si-NC group $(n=5)$ by ANOVA. 

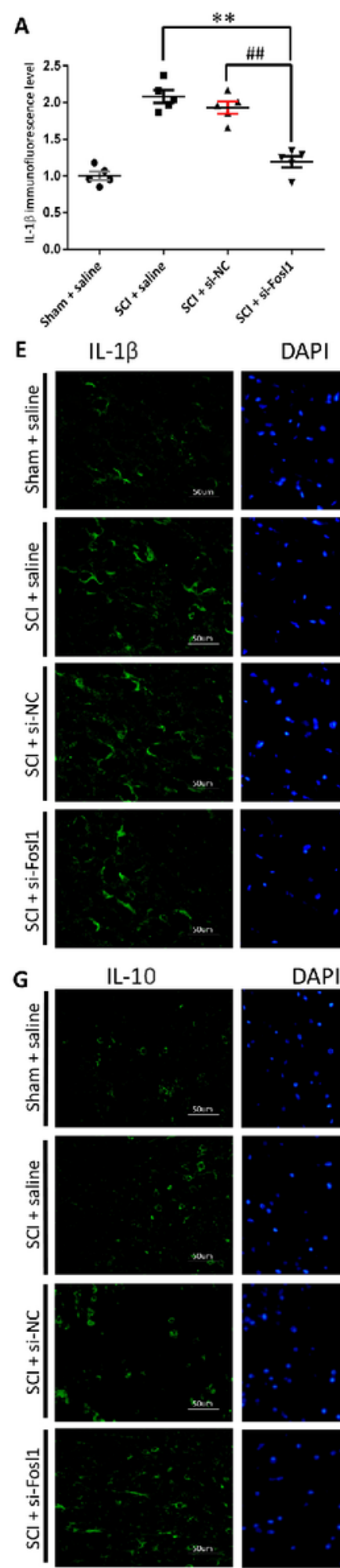

DAPI

DAPI
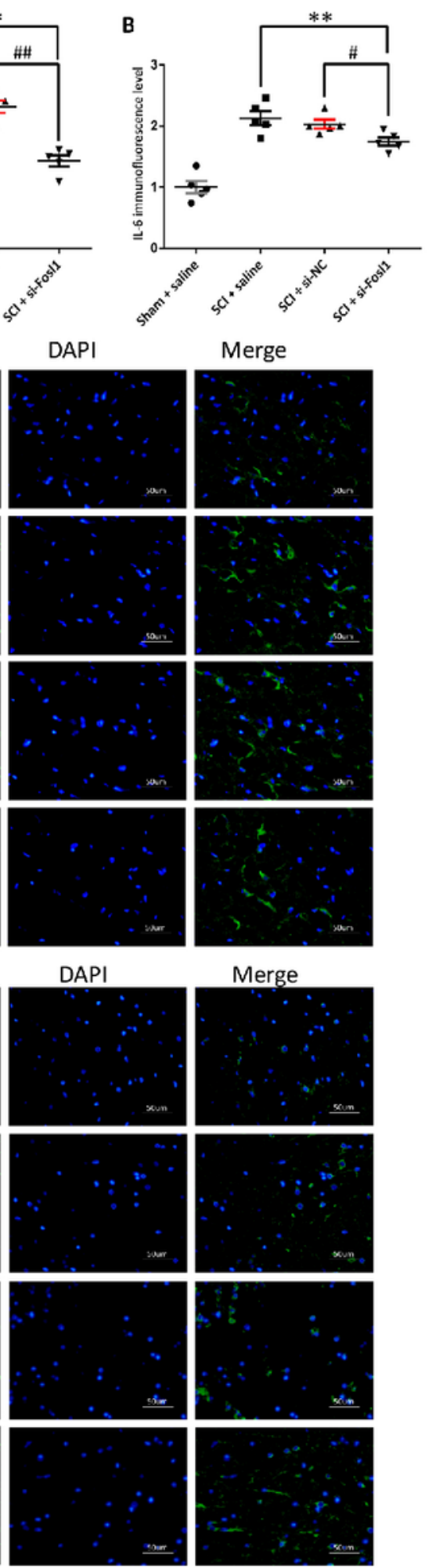

Merge
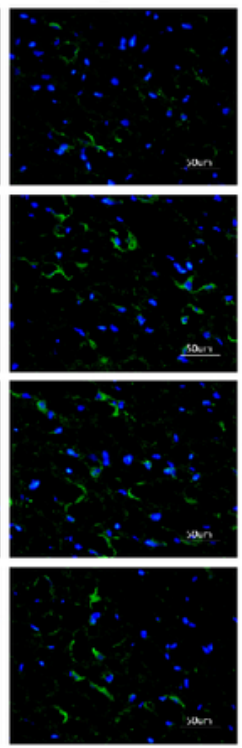

Merge
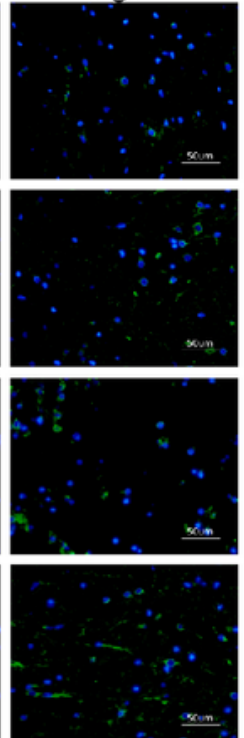
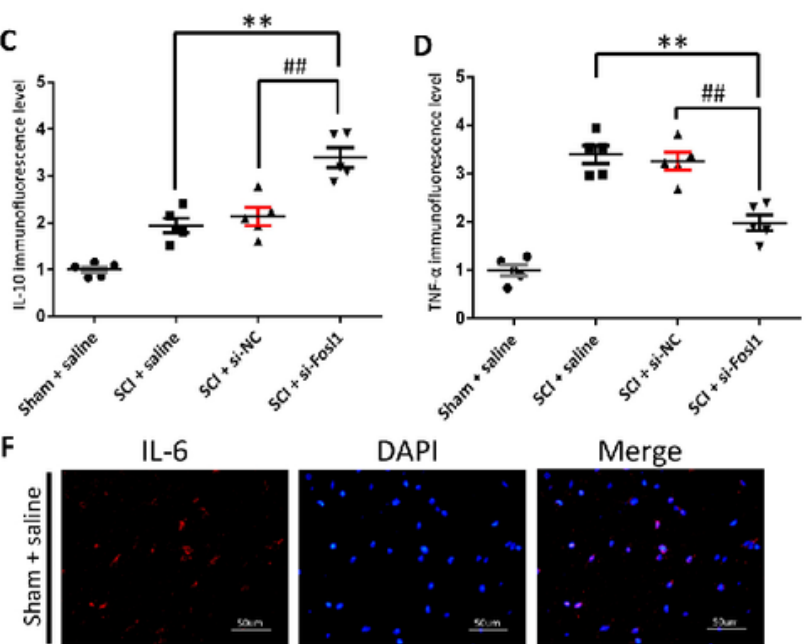

Merge
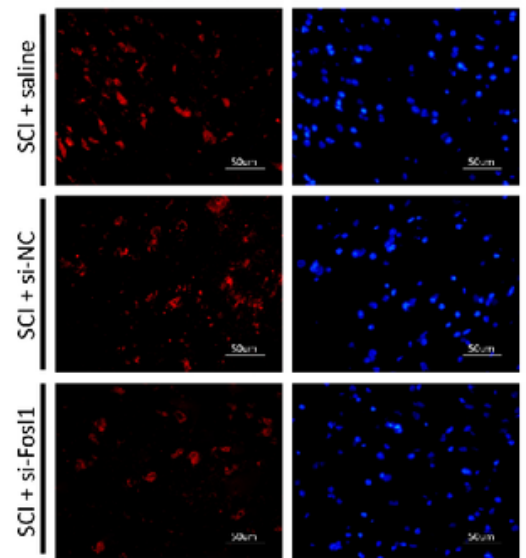

DAPI
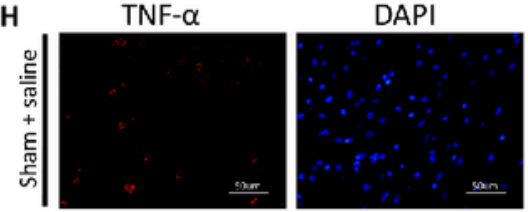

Merge
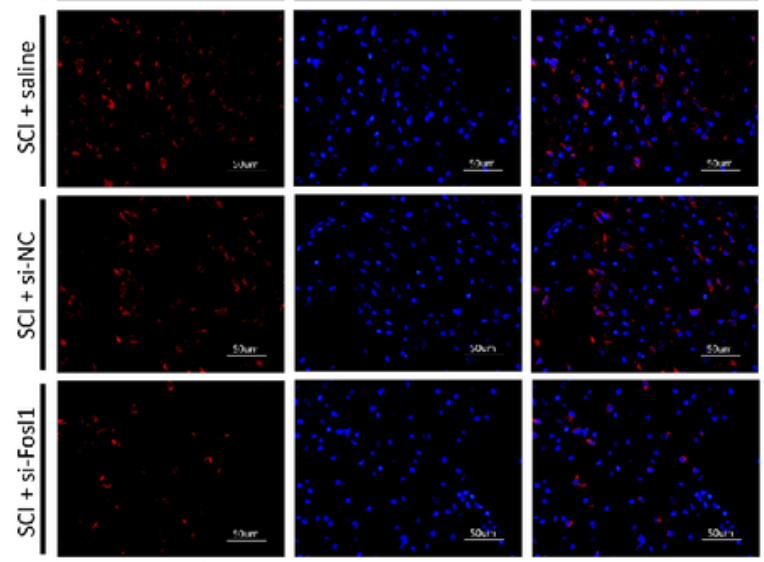

\section{Figure 11}

Fosl1 knockdown downregulated inflammatory cytokines and enhanced anti-ininflammatory cytokine. AD: The histograms of IL-1 $\beta$, IL-6, IL-10 and TNF- $\alpha$ expressions. E: The immunofluorescence staining of IL$1 \beta, I L-6, I L-10$ and TNF- $\alpha$ at $14 d$ (magnification $\times 400)$. All data are presented as the $M \pm S E M(n=5$ in each group). ${ }^{*} p<0.05 \mathrm{vs} . \mathrm{SCl}+$ saline group, ${ }^{\star *} p<0.01 \mathrm{vs}$. $\mathrm{SCl}+$ saline group $(n=5) . \# p<0.05 \mathrm{vs}$. $\mathrm{SCl}+\mathrm{si}-$ NC group, \#\#p<0.01 vs. SCI + si-NC group $(n=5)$ by ANOVA. 
Page 64/64 